\title{
Classification of the Mediterranean lowland to submontane pine forest vegetation
}

\author{
Gianmaria Bonari $^{1,2}$ (1) | Federico Fernández-González ${ }^{3}$ (D) | Süleyman Çoban ${ }^{4}$ (i) | \\ Tiago Monteiro-Henriques ${ }^{5,6}$ (D) | Erwin Bergmeier ${ }^{7}$ (D) | Yakiv P. Didukh ${ }^{8}$ (D) | \\ Fotios Xystrakis ${ }^{9}$ (I) | Claudia Angiolini ${ }^{10}$ (D) | Kryštof Chytrý ${ }^{1}$ (D) | Alicia T.R. Acosta ${ }^{11}$ \\ Emiliano Agrillo $^{12}$ (D) | José C. Costa ${ }^{13}$ (D) | Jiř́ Danihelka ${ }^{1}$ (D) | Stephan M. Hennekens ${ }^{14}$ \\ Ali Kavgaci ${ }^{15}$ (D) | |lona Knollová ${ }^{1}$ (D) | Carlos S. Neto ${ }^{16}$ (D) | Coşkun Sağlam ${ }^{17}$ (i) | \\ Željko Škvorc ${ }^{18}$ (i) | Lubomír Tichý ${ }^{1}$ (i) | Milan Chytrý ${ }^{1}$ (D)
}

${ }^{1}$ Department of Botany and Zoology, Faculty of Science, Masaryk University, Brno, Czech Republic

${ }^{2}$ Faculty of Science and Technology, Free University of Bozen-Bolzano, Bolzano, Italy

${ }^{3}$ Department of Environmental Sciences, University of Castilla-La Mancha, Toledo, Spain

${ }^{4}$ Department of Silviculture, Faculty of Forestry, Istanbul University-Cerrahpaşa, Istanbul, Turkey

${ }^{5}$ Centre for the Research and Technology of Agro-Environmental and Biological Sciences (CITAB), University of Trás-os-Montes and Alto Douro, Vila Real, Portugal

${ }^{6} \mathrm{Global}$ Change and Conservation Lab, Faculty of Biological and Environmental Sciences, University of Helsinki, Finland

${ }^{7}$ Albrecht von Haller Institute of Plant Sciences, University of Göttingen, Germany

${ }^{8}$ Department of Geobotany and Ecology, M.G. Kholodny Institute of Botany, Kyiv, Ukraine

${ }^{9}$ Forest Research Institute, Hellenic Agricultural Organization "Demeter", Vasilika, Greece

${ }^{10}$ Department of Life Sciences, University of Siena, Italy

${ }^{11}$ Department of Sciences, Roma Tre University, Rome, Italy

${ }^{12}$ Institute for Environmental Protection and Research - ISPRA, Rome, Italy

${ }^{13}$ Higher Institute of Agronomy, University of Lisbon, Portugal

${ }^{14}$ Wageningen Environmental Research, Wageningen, The Netherlands

\section{Abstract}

Aim: Vegetation types of Mediterranean thermophilous pine forests dominated by Pinus brutia, Pinus halepensis, Pinus pinaster and Pinus pinea were studied in various areas. However, a comprehensive formal vegetation classification of these forests based on a detailed data analysis has never been developed. Our aim is to provide the first broad-scale classification of these pine forests based on a large data set of vegetation plots.

Location: Southern Europe, North Africa, Levant, Anatolia, Crimea and the Caucasus. Methods: We prepared a data set of European and Mediterranean pine forest vegetation plots. We selected 7,277 plots dominated by the cold-sensitive Mediterranean pine species Pinus brutia, Pinus halepensis, Pinus pinaster and Pinus pinea. We classified these plots using TWINSPAN, interpreted the ecologically and biogeographically homogeneous TWINSPAN clusters as alliances, and developed an expert system for automatic vegetation classification at the class, order and alliance levels.

Results: We described Pinetea halepensis as a new class for the Mediterranean lowland to submontane pine forests, included in the existing Pinetalia halepensis order, and distinguished 12 alliances of native thermophilous pine forests, including four newly described and three informal groups merging supposedly native stands and old-established plantations. The main gradients in species composition reflect elevational vegetation belts and the west-east, and partly north-south, biogeographical differences. Both temperature and precipitation seasonality co-vary with these gradients.

Conclusions: We provide the first formal classification at the order and alliance levels for all the Mediterranean thermophilous pine forests based on vegetation-plot data. This classification includes traditional syntaxa, which have been critically revised, and a new class and four new alliances. We also outline a methodological workflow 
${ }^{15}$ Forestry Faculty, Karabuk University, Karabuk, Turkey

${ }^{16}$ Institute of Geography and Spatial Planning, University of Lisbon, Portugal

${ }^{17}$ Çumra School of Applied Science, Selçuk University, Konya, Turkey

${ }^{18}$ Faculty of Forestry, University of Zagreb, Croatia

\section{Correspondence}

Gianmaria Bonari, Faculty of Science and Technology, Free University of BozenBolzano, Piazza Università 5, I-39100 Bolzano, Italy.

Email: gianmaria.bonari@unibz.it

\section{Funding information}

GB was funded by the post-doc programme of the Department of Botany and Zoology, Masaryk University and by the Free University of Bozen-Bolzano through the CONplant project (TN201H), JD, IK, LT and $\mathrm{MC}$ by the Czech Science Foundation (19-28491X), FFG by the Plan Propio of the UCLM (2020-GRIN-29214), TMH by the European Social Fund (POCH), by National Funds (MCTES) through a Fundação para a Ciência e a Tecnologia postdoctoral fellowship (SFRH/BPD/115057/2016) and project no. UIDB/04033/2020.

Co-ordinating Editor: Jörg Ewald that might be useful for other vegetation classification syntheses. The expert system, which is jointly based on pine dominance and species composition, is a tool for applying this classification in research and nature conservation survey, monitoring and management.

\section{KEYWORDS}

biogeography, classification, expert system, forest, Mediterranean Basin, phytosociology, pine, Pinetea halepensis, Pinus, Quercetea ilicis, vegetation classification, vegetation-plot database

\section{1 | INTRODUCTION}

Mediterranean coniferous tree taxa are included in the genera Abies, Cedrus, Cupressus, Juniperus, Pinus, and Tetraclinis. Especially Pinus, the most species-rich genus of conifers, is abundant across southern Europe and the Mediterranean Basin (Timbal et al., 2005; Farjon \& Filer, 2013). There are ten pine tree species currently accepted in Europe and North Africa (Euro+Med, 2016-2020) and several infraspecific taxa. Four pine species can be considered Mediterranean in terms of their temperature requirements and distribution, which is confined to low- to mid-elevations: Pinus brutia, Pinus halepensis, Pinus pinaster and Pinus pinea (Barbéro et al., 1998; Quézel, 2000). Their distribution range lies mainly in the countries bordering the Mediterranean Sea, partly the Black Sea (Pinus brutia), and the Atlantic coast of the lberian Peninsula and Morocco (Pinus pinaster, Pinus pinea; Caudullo et al., 2017). Marginally, Pinus halepensis is also present in the Atlantic watersheds of the Iberian Peninsula and Morocco (Caudullo et al., 2017).

Specifically, Pinus brutia occurs in the east of the Mediterranean Basin and some areas around the Black Sea (Crimea, northwestern Caucasus, eastern foothills of the Great Caucasus and isolated areas in the Euxinian region), Iraq and Iran (Mauri et al., 2016; Çoban \& Willner, 2019). It occurs chiefly in the thermo- and mesomediterranean elevational belts, where it forms extensive stands with a dense shrub layer of sclerophyllous species (macchia, maquis), or without shrub layer (Farjon \& Filer, 2013; Bonari et al., 2020).
Pinus halepensis is the most widespread Mediterranean thermophilous pine species (Mauri et al., 2016), but it is more common in the western Mediterranean Basin. In most areas, it occurs not far from the coast but reaches about 2,000 m a.s.I. in the Eastern High Atlas. It occurs in fire-prone vegetation (macchia, garrigue), but can also form a dense canopy with sparse undergrowth in places with a long absence of fire (Farjon \& Filer, 2013). The distribution ranges of Pinus halepensis and Pinus brutia overlap in Greece and on the Aegean islands.

Pinus pinaster thrives in the western Mediterranean Basin (Abad Viñas et al., 2016a). It is a thermophilous species believed to be native to the Iberian Peninsula, southern France including Corsica, western Italy including Sardinia, and northwestern Africa. It also occurs on the Atlantic coast and reaches about 2,000 m a.s.l. in Morocco. This species is well adapted to fire (Fernandes \& Rigolot, 2007; Farjon \& Filer, 2013).

Pinus pinea is an iconic Mediterranean thermophilous tree, which occurs from the sea level up to the mountains. It has been frequently planted as an ornamental tree and for its edible seeds. It occurs at high elevations mainly in the eastern Mediterranean, where it naturally regenerates at some sites (Abad Viñas et al., 2016b).

The current distribution of the Mediterranean pines is influenced by the geological history of the Mediterranean Basin and climatic conditions during the Quaternary (Panetsos, 1981), though there were additional influences by humans. Especially the native 
distribution of Pinus pinea, and to a lesser extent of Pinus pinaster, is uncertain because their current distribution is highly influenced by planting, mainly in the western Mediterranean (Mazzoleni, 2004; Bonari et al., 2020). Humans have always taken advantage of the modest ecological requirements of pines and they have used them extensively in plantations for centuries (Bonari et al., 2017), although on the Iberian Peninsula most of the pine plantations were established only in the 20th century. Plantations are easily identified if they occur outside the native distribution range of the dominant pine species, but old plantations in the native range of the pine species may be difficult to distinguish from natural pine forests. Understorey species composition of pine forests varies considerably in response to many factors, including management and disturbances (Farjon \& Filer, 2013; Kavgacı et al., 2017; Bonari et al., 2019a).

According to phylogenetic analyses (Gernandt et al., 2005), these four pine species (Pinus brutia, Pinus halepensis, Pinus pinaster and Pinus pinea) belong to the section Pinus, subsect. Pinaster, which has a mesogean distribution (Western Himalayas, Mediterranean region and Macaronesia), while the other European species belong to the subsect. Pinus or the subgenus Strobus (section Quinquefoliae). Pinus heldreichii, occurring in the Balkans and southern Italy, also belongs to subsect. Pinaster but it is a mountain pine. Pinus halepensis and Pinus brutia are more closely related to each other than to the other species of the subsect. Pinaster. Also, Pinus halepensis, Pinus brutia and Pinus pinaster show some adaptations to fire (serotinous cones, early reproductive age) lacking in Pinus pinea.

Besides broad overviews of forest vegetation in the Mediterranean Basin (Scarascia-Mugnozza et al., 2000; Gauquelin et al., 2018), a number of studies specifically addressed pine forest vegetation diversity in this area. Recent research focused on understanding pine forest understories and their dynamics (Zavala \& Zea, 2004; Madrigal-González et al., 2010; Bonari et al., 2017), their habitat types (Bonari et al., 2018) and legislative tools for their conservation (Leone \& Lovreglio, 2004). Forestry management practices in pine forests have also been in the spotlight (Granados et al., 2016; Martínez-Jauregui et al., 2018; Bonari et al., 2019a). While some studies tried to clarify the native range of pine forests (Martínez \& Montero, 2004; Bonari et al., 2020), others focused on the plantations and their dynamics, for instance, the expansion of Mediterranean pines from plantations into adjacent natural non-forest plant communities (Lavi et al., 2005). Effects of pine plantations on soil, faunal communities, vegetation, biotic and abiotic gradients were also reviewed (Maestre \& Cortina, 2004; Gómez-Aparicio et al., 2009).

The most important contributions to the syntaxonomy of the Mediterranean pine forests so far were made by French authors, especially Pierre Quézel and Marcel Barbéro, who performed extensive field surveys in the Mediterranean Basin from the 1970s to the 1990s. They focused mainly on the eastern Mediterranean Basin (Quézel et al., 1978), including different parts of Anatolia (Quézel \& Pamukçuoǧlu, 1973; Akman et al., 1978, 1979; Quézel et al., 1980), Syria (Barbéro et al., 1976), Lebanon (Chouchani et al., 1974; Abi-Saleh et al., 1976), Greece (Barbéro \& Quézel, 1976) and
Cyprus (Barbéro \& Quézel, 1979), but also North Africa (Quézel \& Barbéro, 1976; Barbéro et al., 1981; Quézel et al., 1987, 1988, 1992; Benabid, 1988) and France (Quézel \& Barbéro, 1988). These contributions have created a backbone for the syntaxonomical scheme of the Mediterranean thermophilous pine forests for a long time, although significant advances have been achieved since then. The most recent comprehensive classification of European vegetation, EuroVegChecklist (Mucina et al., 2016), included the Mediterranean pine forests in the classes of broad-leaved forests, Quercetea ilicis and Quercetea pubescentis, following the established tradition (Barbéro et al., 1974; Rivas-Martínez, 1974; Quézel \& Barbéro, 1986; Rivas-Martínez et al., 1986; Brullo et al., 2008).

There are open questions of paramount importance for the Mediterranean pine forests and their management, such as climate change effects, fire risk, or the dynamics of alien plant invasions. This research agenda for the near future, as well as conservation planning and management, can be significantly supported by a welltested classification scheme for the Mediterranean pine forest types. Widely conflicting views on the syntaxonomy of the Mediterranean pine forests still exist even after the publication of EuroVegChecklist (Mucina et al., 2016) because the alliances accepted in this checklist have never been tested with a comprehensive set of vegetation-plot data. Moreover, forests dominated by Mediterranean pines were, at least in the past, not identified as independent syntaxa in spite of their distinct physiognomy and their wide distribution across the Mediterranean Basin. Due to the frequent presence of many macchia species, they were relegated into shrubland vegetation units of the order Pistacio lentisci-Rhamnetalia alaterni. For example, RivasMartínez et al. (1986) listed Pinus halepensis as a character species of this order. This is not consistent with the treatment of boreal or temperate pine forests which are classified in different classes than the broad-leaved forests and shrublands. This approach was partly inherited from the view of early researchers who considered the pine forests as non-climax vegetation. However, at least Pinus brutia and Pinus halepensis can form pure climax forests in a suitable climate (Feinbrun, 1959; Quézel, 2000; Boydak, 2004; Bonari et al., 2020). Another explanation lies in the fact that the native distribution of some pine species is contentious (see e.g. Martínez \& Montero, 2004), and some of the extant pine forests may have originated as ancient plantations. This may be the cause for the reluctance of phytosociologists to describe syntaxa based on dominant species of uncertain origin. With increasing knowledge of the distribution of both species and communities, new syntaxonomical units of Mediterranean thermophilous pine forests were described in recent years (e.g. Pérez Latorre et al., 1998; Mucina et al., 2009; Biondi et al., 2014; Biondi \& Vagge, 2015; Mucina et al., 2016; Pesaresi et al., 2017).

Currently, interest in vegetation classification and its applications is growing (Biurrun et al., 2019). The introduction of new numerical methods and formal classification approaches (De Cáceres et al., 2015) and the availability of large vegetation-plot databases (Dengler et al., 2011; Chytrý et al., 2016; Bruelheide et al., 2019) 
contributed to overcoming the criticism of subjectivity of the traditional Braun-Blanquet method of vegetation classification (Braun-Blanquet, 1932). This has also paved the way for synthetic international vegetation classification studies on the European scale (e.g. Douda et al., 2016; Peterka et al., 2017; Willner et al., 2017a; Marcenò et al., 2018, 2019; Landucci et al., 2020). In our case, data from the European Vegetation Archive (EVA; Chytrý et al., 2016) and from the specialized CircumMed Pine Forest Vegetation Database (Bonari et al., 2019b) made it possible to perform a detailed analysis of the Mediterranean pine forests and to accomplish the revision of their classification.

Our aim is to characterize the general diversity of pine forests in the Mediterranean Basin by providing the first comprehensive and internally consistent international classification consensus for the Mediterranean thermophilous low-elevation pine forest types at the alliance level across the Mediterranean Basin and the Black Sea region, based on an analysis of vegetation-plot data.

\section{2 | METHODS}

\section{1 | Study area}

The study area is the Mediterranean Basin and adjacent areas, broadly corresponding to the oceanic Mediterranean bioclimates as defined and mapped by Rivas-Martínez and Rivas Sáenz (2019) for Eurasia and North Africa. It stretches from the Atlantic coasts of Portugal to easternmost Anatolia, measuring approximately $4,300 \mathrm{~km}$ along its broadest longitudinal extent $\left(9^{\circ} \mathrm{W}-42^{\circ} \mathrm{E}\right)$, and from the Caucasus to Palestine to southern Morocco, extending approximately $1,300 \mathrm{~km}$ along its broadest latitudinal extent $\left(48^{\circ} \mathrm{N}-30^{\circ} \mathrm{N}\right)$. We considered all the countries bordering the Mediterranean Sea, as well as Portugal, Crimea, the Caucasus and the Euxinian region fringing the southern coast of the Black Sea. The latter three territories were included because of the disjunct native occurrence of Pinus brutia. In the northern part of the range of these forests, orographic features of high mountain ranges protect them from the effects of northerly winds. In addition, the proximity to the Black Sea raises air moisture and precipitation, contrasting with the arid and more continental climates of the surrounding areas. This causes the extension of the distribution range of Pinus brutia forests and many Mediterranean species, which reach as far north as Crimea and the foothills of the Great Caucasus (Didukh, 1992).

The physical-geographic complexity of the Mediterranean Basin needs to be taken into account when dealing with biological communities. Firstly, the Mediterranean Basin encompasses a high number of bedrock types. Limestone is by far the most common, while areas with acidic bedrock are scattered, although locally abundant. Ultramafic rock patches are also present. Bedrock diversity translates into soil diversity (Blondel et al., 2010) and thus into vegetation diversity.

Secondly, the specific Mediterranean climate is characterized by mild, wet winters and warm, dry summers. Temperatures generally increase from north to south. Mean temperatures of the summer months exceed $22{ }^{\circ} \mathrm{C}$ but are above $30^{\circ} \mathrm{C}$ in some areas. Summers are characterized by the lack of rain, which in combination with high temperatures leads to marked seasonal aridity. The limited occurrence of winter frost is essential for plants. The total annual precipitation is spatially highly variable, ranging from less than $200 \mathrm{~mm}$ in North Africa to $2,000 \mathrm{~mm}$ in some northern mountainous areas (Lionello, 2012; Rundel et al., 2016).

Thirdly, numerous mountain ranges around the Mediterranean Basin show distinct elevational vegetation belts (Ozenda, 1975; Quézel, 1979; Rivas-Martínez, 1981; Blondel et al., 2010). Different pine species tend to occur at different elevations, although with some overlaps (e.g. Carrión et al., 2000). This allows a clear ecological distinction between two major groups of tree pines in the Mediterranean Basin: Mediterranean thermophilous species (Pinus brutia, Pinus halepensis, Pinus pinaster and Pinus pinea) and mountain species (Pinus cembra, Pinus heldreichii, Pinus nigra, Pinus peuce, Pinus sylvestris and Pinus uncinata). This study focuses on the former group.

\subsection{Data set and its standardization}

The workflow of this study is summarized in Figure 1. We requested vegetation plots (phytosociological relevés) from EVA (Chytrý et al., 2016) from the following countries: Albania, Andorra, Austria, Belgium, Bosnia-Herzegovina, Bulgaria, Croatia, Cyprus, Czech Republic, France, Germany, Greece, Hungary, Italy, Kosovo, Luxemburg, Macedonia, Malta, Moldova, Monaco, Montenegro, The Netherlands, Poland, Portugal, Romania, San Marino, Serbia, Slovakia, Slovenia, Spain, Switzerland, Turkey, Ukraine (only the Carpathians and Crimea), North African countries (Algeria, Morocco, Tunisia), eastern Mediterranean countries (Israel, Lebanon, Syria), Georgia and Russia south of $45^{\circ} \mathrm{N}$. The plots had to contain at least one of the following species (including all of their subspecies and varieties) with a cover value $\geq 15 \%$ : Pinus brutia, Pinus cembra, Pinus halepensis, Pinus heldreichii, Pinus nigra, Pinus peuce, Pinus pinaster, Pinus pinea, Pinus sylvestris and Pinus uncinata. We used a cover threshold of $15 \%$ to exclude very open vegetation with the presence of pines, but at the same time to include plots that represented forests and open pine woodlands. For a better delimitation of the vegetation types of interest, we also included vegetation with other relevant Mediterranean conifers and Mediterranean oaks (including all of their subspecies and varieties) with a cover value $\geq 30 \%$ : Abies borisii-regis, Abies cephalonica, Abies cilicica, Abies marocana, Abies nebrodensis, Abies nordmanniana, Abies numidica, Abies pinsapo, Cedrus atlantica, Cedrus libani, Cupressus sempervirens, Juniperus drupacea, Juniperus excelsa, Juniperus foetidissima, Juniperus thurifera, Tetraclinis articulata, Quercus coccifera, Quercus ilex, Quercus rotundifolia and Quercus suber. Further, we included data of the pine forest-focused CircumMed Pine Forest Vegetation Database (Bonari et al., 2019b), formerly prepared to fill the gaps in EVA in pine forest-vegetation data. We thus obtained 63,138 vegetation plots in total.

The data set contained vegetation plots sampled over several decades by many authors from different countries who used different flora 


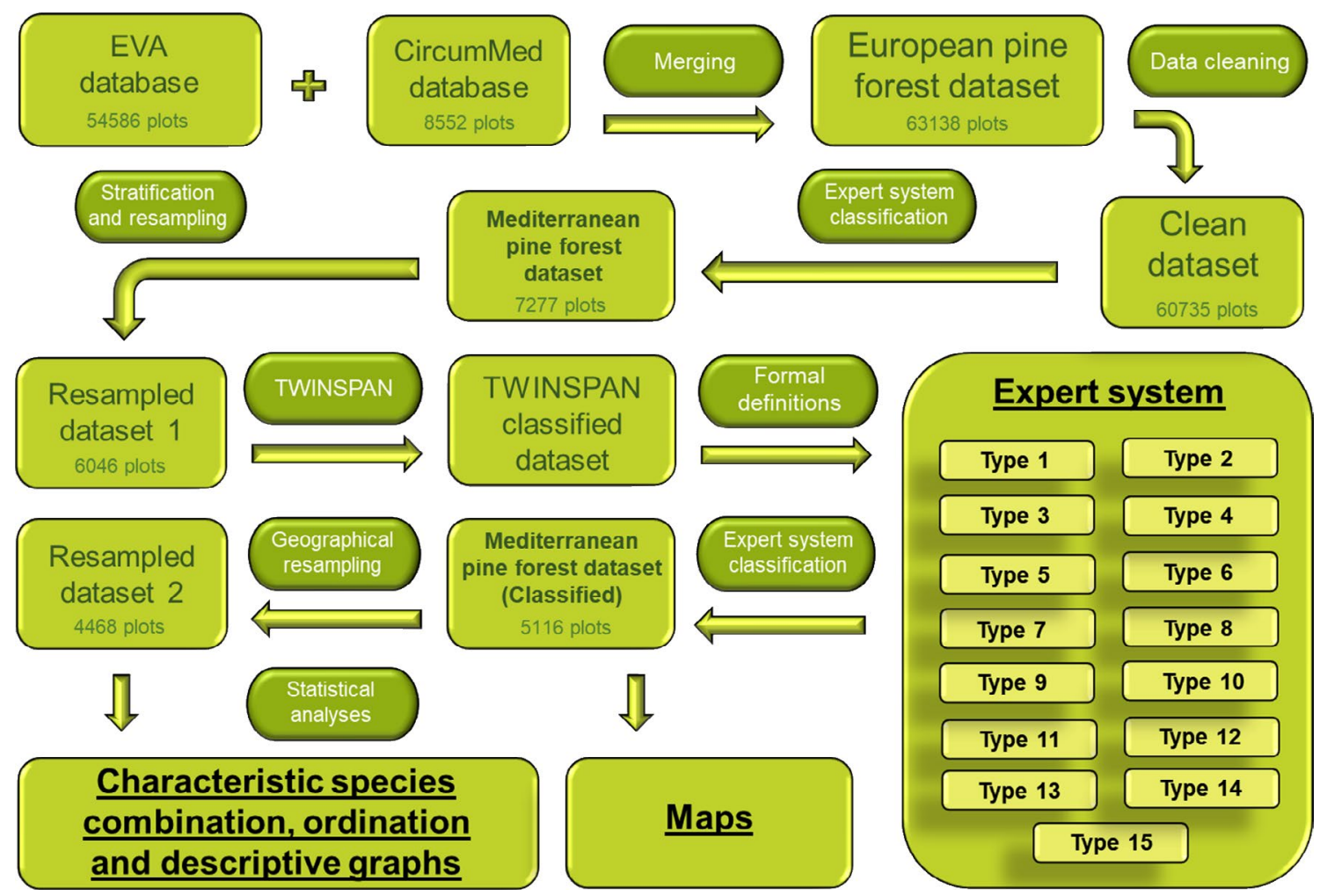

FIGURE 1 Workflow adopted in this study showing the steps from data set creation to the results (underlined), including vegetation types, characteristic species combination, maps, boxplots and elevational-density graphs. EVA = European Vegetation Archive;

CircumMed database = CircumMed Pine Forest Vegetation Database; Ordination = DCA ordination superimposed with climatic variables; Plots $=$ vegetation plots. The extraction of 5,000 random plots from the clean data set for the distinction of low-elevation pine forests from the other pine forests (see paragraph 2.2) and the classification of plots with the EUNIS expert system for the most frequent species of Quercetea ilicis and Pinetea halepensis (see paragraphs 2.4 and 3.1) are not shown in the workflow

manuals and taxon concepts. We used the SynBioSys Taxon Database in TURBOVEG 3, which matches the taxon concepts and unifies the taxon names used in different databases included in EVA (Chytrý et al., 2016). Subsequently, we adjusted the taxonomy and nomenclature to the Euro+Med PlantBase (Euro+Med, 2016-2020). The few taxa not included in Euro+Med were named according to the SynBioSys Taxon Database or using the original names given in the source publications or in individual EVA databases. The taxa recorded with different taxonomic resolution were merged into aggregates (e.g. Achillea millefolium aggr., Centaurea alba aggr., Draba verna aggr., Galium mollugo aggr.). Pines were considered at the species level because subspecies were not always identified in the data set. Also, especially for Pinus pinaster, there is no taxonomic agreement among authors about its subspecies.

Further, we reduced the noise and inconsistencies in the data as follows: (1) bryophytes, lichens and algae were excluded, because they were present only in a subset of vegetation plots; (2) infraspecific taxa were merged into species; (3) species with less than five occurrences in the data set were deleted; (4) tree and shrub species recorded in the herb layer or marked as seedlings or juveniles were deleted; (5) records of the same species in different layers were merged into a single layer; (6) vegetation plots with a size $<50 \mathrm{~m}^{2}$ or $>1,000 \mathrm{~m}^{2}$ were excluded, but plots without size information were retained assuming that most of them were within this size range.
These steps created a data set of 60,735 vegetation plots. The data cleaning was done using the JUICE program v. 7.1 (Tichý, 2002).

To test the differentiation between the Mediterranean thermophilous and non-thermophilous pine forests, and between Mediterranean pine forests and Mediterranean oak forests, we performed an unsupervised classification of the whole data set using TWINSPAN (Hill, 1979; parameters: three pseudospecies cut levels of species percentage cover: $0 \%, 10 \%, 25 \%$; minimum group size for division: 10 plots) on a subset of 5,000 plots that were randomly chosen to meet the technical limit of the number of plots that the TWINSPAN program could process. The result is shown in Table 1. Column 1 of this table groups Mediterranean lowland to submontane pine forests (with Pinus brutia, Pinus halepensis, Pinus pinaster and Pinus pinea), oak forests ( $42 \%$ constancy of Quercus ilex) and other Mediterranean forests. These forests strongly differ from the northern and mountain pine forests. Only Pinus nigra has a transitional distribution between these two groups, but it is more mountainous than the four thermophilous pines. The analysis gives support to the separation in the first division between the Mediterranean thermophilous pine forests and the other pine forests, but not to the separation between Mediterranean pine vs. oak forests (Tables 1 and 2). The floristic criterion used by TWINSPAN does not support this division, which can nevertheless 
TABLE 1 Shortened synoptic table showing the result of a TWINSPAN classification into two groups of a random selection of 5,000 plots from the initial data set of the Mediterranean pine forest and their related forest types including evergreen oak forests; the numbers in columns 1 and 2 are percentage constancies and points represent species absence; the species shown include the pine species and five other species with the highest value of the phi coefficient $(\Phi)$ for one of the two groups; grey shading represents species with $\Phi>0.2$, Constancy Ratio (CR) $>1.5$ and $p<0.05$ (based on Fisher's exact test)

\begin{tabular}{|c|c|c|c|}
\hline Group & No. of plots & 1 & 2 \\
\hline No. of plots & & 2,062 & 2,938 \\
\hline \multicolumn{4}{|l|}{ Species } \\
\hline Pinus halepensis & 422 & 20 & . \\
\hline Pinus pinaster & 248 & 11 & . \\
\hline Pinus brutia & 190 & 9 & . \\
\hline Pinus pinea & 101 & 5 & . \\
\hline Pinus sylvestris & 248 & 1 & 83 \\
\hline Pinus nigra & 591 & 9 & 14 \\
\hline Pinus uncinata & 91 & . & 3 \\
\hline Pinus mugo aggr. & 59 & . & 2 \\
\hline Pinus cembra & 59 & . & 2 \\
\hline Pinus heldreichii & 25 & . & 1 \\
\hline Pinus peuce & 19 & 1 & 1 \\
\hline Pinus $\times$ rhaetica & 8 & . & 1 \\
\hline Rubia peregrina & 102 & 48 & 1 \\
\hline Quercus ilex & 898 & 42 & 1 \\
\hline Asparagus acutifolius & 679 & 33 & 1 \\
\hline Smilax aspera & 633 & 31 & 1 \\
\hline Phillyrea latifolia & 605 & 29 & 1 \\
\hline Vaccinium myrtillus & 127 & . & 43 \\
\hline Avenella flexuosa & 120 & 1 & 41 \\
\hline Sorbus aucuparia & 111 & 1 & 38 \\
\hline Quercus robur & 108 & 1 & 36 \\
\hline Betula pendula & 907 & 1 & 31 \\
\hline
\end{tabular}

be based on the stand physiognomy (dominance of conifers vs. broad-leaved evergreen trees).

\section{3 | Mediterranean thermophilous low-elevation pine-forest data set and resampling}

As the TWINSPAN classification showed that the vegetation of forests dominated by the four low- to mid-elevation Mediterranean pines (Pinus brutia, Pinus halepensis, Pinus pinaster and Pinus pinea) differs from the mountain and temperate forests dominated by other pine species (paragraph 2.2), we analysed these forests separately (hereafter for short referred to as Mediterranean pine forests). From the total data set of 60,735 vegetation plots, we selected those in which the total cover of the four Mediterranean pine species was greater than or equal to $15 \%$ and exceeded the total cover of the other trees. Where information was available, we excluded vegetation plots sampled in recent plantations located clearly out of the alleged native distribution range of the dominant pine species, while we retained those from putative old-established plantations. Note that it is often not possible to separate native stands from old plantations, especially in the Mediterranean Basin where humans have been changing the landscape for millennia. Delineating what is natural and what is not is even more complicated when working with large vegetation-plot databases, in which more detailed information on individual plots is often missing. The selection resulted in a data set of 7,277 Mediterranean pine forest plots (Figure 2). The contributions from individual databases are reported in Appendix S1.

At this stage, we removed 381 plots with no coordinates. Vegetation plots with available coordinates ( $n=6,896$; Figure 2 ) were assigned to cells of a geographic grid of 0.6 longitudinal by 0.45 latitudinal minutes, i.e. approximately $50 \mathrm{~km} \times 50 \mathrm{~km}$ in the central part of the study area. Subsequently, we performed a geographical resampling in order to overcome the bias due to uneven sampling density across the study area (Knollová et al., 2005). We resampled cells with more than 10 plots per grid cell. This operation removed 650 vegetation plots. In the grid cells that contained more than ten plots, we applied the Heterogeneity-Constrained Random (HCR) resampling algorithm (Lengyel et al., 2011) calculated with Bray-Curtis dissimilarity in plot species composition. This procedure guaranteed that the resampled data set contained, within each cell, plots that were representative of the variation in species composition within that cell. This operation removed 200 vegetation plots. The final data set was a matrix of 6,046 plots and 3,190 taxa (hereafter called "resampled data set" and "Resampled data set 1" in Figure 1). Resampling was performed using JUICE v. 7.1 (Tichý, 2002).

\subsection{Classification and determination of diagnostic species}

First, unsupervised divisive classification of the resampled data set was performed using TWINSPAN (Hill, 1979; parameters: three pseudospecies cut levels of species percentage cover: 0\%, $10 \%, 25 \%$; minimum group size for division: 10 plots). Four division levels were used, resulting in 16 clusters. This operation allowed us to understand the coarse patterns of floristic similarity within our data set. With a few exceptions, each cluster contained plots dominated by a single pine species. When no ecologically or biogeographically interpretable dissimilarities in species composition between clusters were found, these clusters were merged. We also interpreted all the clusters syntaxonomically, comparing their floristic, ecological and biogeographical characteristics with the literature. The aim was to identify previously described alliances in our TWINSPAN groups. When the analysis supported the concepts proposed in the literature, we accepted those concepts, meaning that we took a conservative approach. When establishment of a new vegetation unit appeared to be necessary, we considered not 


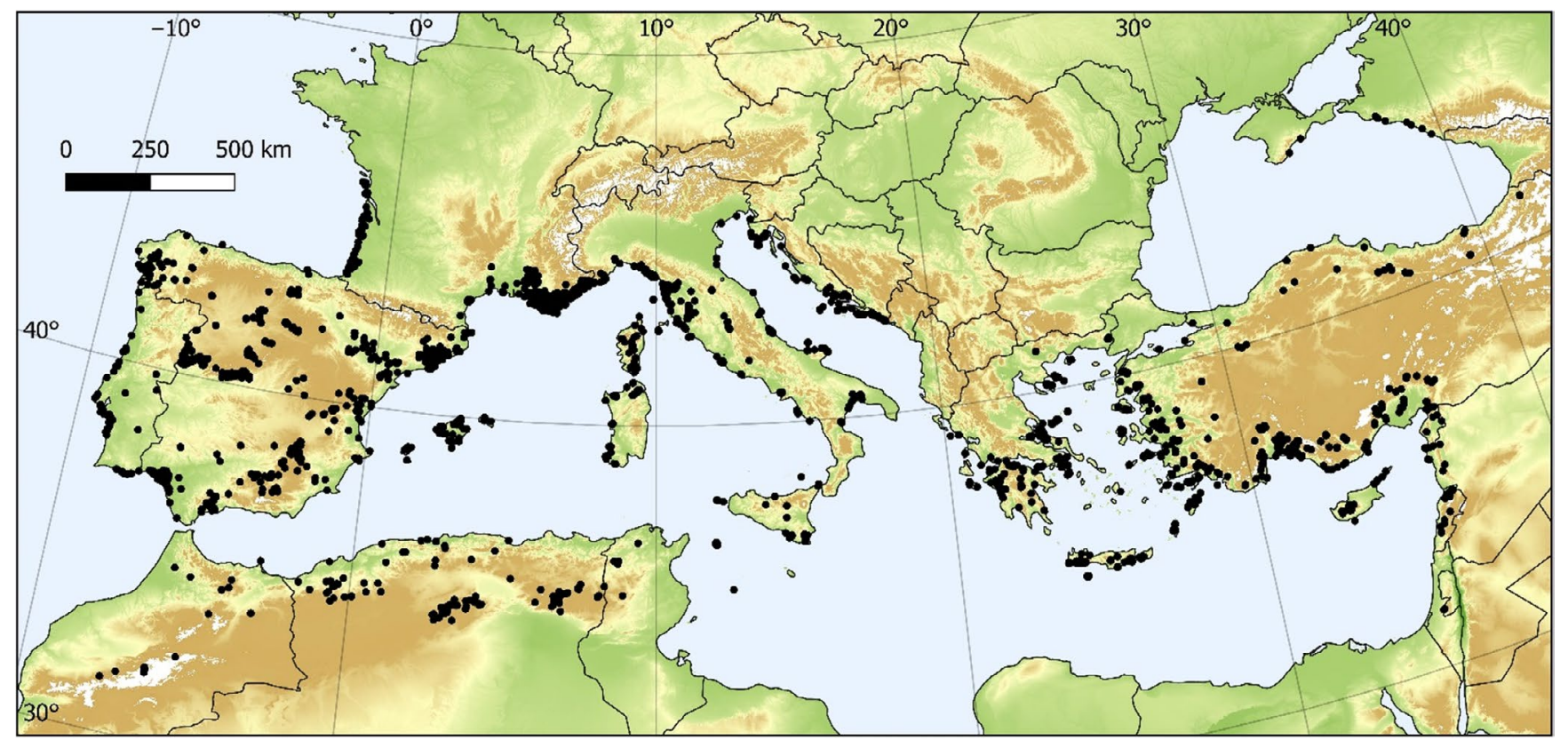

FIGURE 2 A map of 6,896 vegetation plots used in this study. Each of them is dominated by one of the four Mediterranean thermophilous low-elevation pines (Pinus brutia, Pinus halepensis, Pinus pinaster, Pinus pinea). Plots with no coordinates $(n=381)$ are not shown

only floristical but also ecological and biogeographical differences from the already established units. We also accepted two types (see paragraphs 3.1.3 and 3.1.4) that did not appear as distinct clusters in the TWINSPAN classification, given the scarcity of plots of these types in the database. We defined them by means of the expert system only. All the analyses were performed in JUICE v. 7.1 (Tichý, 2002). Phytosociological nomenclature is in agreement with the fourth edition of the International Code of Phytosociological Nomenclature (ICPN; Theurillat et al., 2021).

Formal definitions of syntaxa provide reproducible and unambiguous classification (e.g. Chytrý et al., 2020). We prepared formal definitions of the interpreted alliances and informal vegetation types based on the concept of functional species groups (Landucci et al., 2015; Tichý et al., 2019) linked by the logical operators AND, OR and NOT as proposed by Bruelheide (1997). Diagnostic species, determined based on the calculation of the phi coefficient of association $(\Phi)$, were calculated for the TWINSPAN-based clusters and used to create the functional species groups and discriminating species groups to be used in the formal definitions. Some of these groups were improved by adding a few species on the basis of expert knowledge. The phi coefficient of association was used as a fidelity measure and calculated for equalized size of clusters following Tichý and Chytrý (2006). We included formal definitions into a classification expert system that is available as TXT file (Appendix S2; for acronyms of vegetation types see paragraphs 3.1.1-3.1.15) and can be run in JUICE v. 7.1 (Tichý, 2002), TURBOVEG 3 (Hennekens, 2015) or R (Bruelheide et al., https://git.loe.auf.uni-rostock.de/misc/ESy)

We determined diagnostic species of individual alliances based on the data set resampled within grid cells defined as above, but this time nested within alliances ("Resampled data set 2" in Figure 1), meaning that unlike in the "Resampled data set 1, " where the geographical resampling was applied to the whole matrix, here this operation was done within the defined alliances to produce reliable diagnostic species. For each alliance or informal group, we resampled cells with more than 10 plots per grid cell. We defined diagnostic species for a particular vegetation type as species with $\Phi \geq 0.2$, Fisher's exact test $p$ value of the probability of the given concentration of species occurrences within the cluster $<0.05$ and Constancy Ratio $>1.5$. Constancy Ratio is the ratio between species constancy (relative frequency) in the cluster for which the species has the highest constancy and the maximum constancy recorded in any other cluster (Willner et al., 2017b). We defined constant species as those with relative frequency $>20 \%$ and dominant species as those occurring in at least $5 \%$ of plots with a cover $>15 \%$.

Based on "Resampled data set 2," we also prepared the ordination diagram, the elevational-density graph and the boxplots for the recognized alliances and informal groups.

To assess differences in species composition between Mediterranean pine forests and Mediterranean broad-leaved forests, we extracted 1,534 plots classified as "T3A Mediterranean lowland to submontane Pinus forest" and 2,826 vegetation plots as "T21 Mediterranean evergreen Quercus forest" from the EVA database classified by the EUNIS Habitat Classification expert system (EUNIS-ESy v. 2020-06-08; Chytrý et al., 2020). These two habitat types correspond to the classes Pinetea halepensis and Quercetea ilicis, respectively. We identified the species with the highest frequency and calculated their phi coefficient of association for these two habitat types.

All the procedures described in this section were performed using JUICE v. 7.1 (Tichý, 2002). 


\section{5 | Ordination}

To relate the differentiation of the accepted alliances to climate, DCA ordination (Hill \& Gauch, 1980) of plots was computed with log-transformed percentage covers of species using the vegan package (v. 2.5-6; Oksanen et al., 2019) in R (v. 3.6.1; R Core Team, 2019). Individual plot coordinates were overlaid with the CHELSA Bioclim data set v. 1.2 (Karger et al., 2017) using the "envfit" function of the vegan package. Climatic data consist of a downscaled model output with temperature and precipitation estimates at a horizontal resolution of 30 arc-seconds (Karger \& Zimmermann, 2019). Correlations between 19 climatic variables were calculated using the Spearman correlation coefficient (Sokal \& Rohlf, 1995) to reduce the number of available variables. We retained only those variables that were most clearly interpretable from an ecological point of view: mean annual temperature, temperature seasonality (standard deviation of the monthly mean temperatures), annual precipitation and precipitation seasonality (standard deviation of the monthly precipitation estimates expressed as a percentage of the annual mean). The four climatic variables were extracted from vegetation plots with help of the raster package (v. 3.1-5; Hijmans, 2020) using the bilinear method. Apart from the DCA, we displayed the climatic variables in boxplots for each accepted alliance and informal group.

\section{3 | RESULTS}

We interpreted TWINSPAN clusters mainly at the fourth hierarchical level of division (Figure 3) based on species composition, geographic distribution and literature. The first TWINSPAN division mainly separated the eastern and western Mediterranean pine forest communities, suggesting a biogeographic distinction between them. The divisions at the second and third hierarchical levels were mainly based on the dominance of different species of pines and elevational differences, respectively, with partial overlaps between some clusters.

Overall, we recognized 12 alliances and three informal groups of communities of supposedly native forests, including old-established plantations in the TWINSPAN clusters on the third and fourth level of division. A large majority of them were associated with the dominance of one of the four low-elevation Mediterranean pine species (Pinus brutia, Pinus halepensis, Pinus pinaster, Pinus pinea). One alliance (Sarcopoterio spinosi-Pinion halepensis) was identified at the fifth hierarchical level and is not shown in Figure 3. Also, it is worth mentioning that although many species of Quercetea pubescentis are present in the plots from the coastal areas of the northern Black Sea, TWINSPAN did not separate these plots, most likely due to their very low proportion in the data set. Therefore, these two small clusters (Jasmino fruticantis-Juniperion excelsae and Campanulo sibiricae-Pinion brutiae) represented by a few plots were separated in the expert system. However, most of the TWINSPAN clusters were accepted, either stand-alone or merged, as alliances or informal groups. When a given cluster was split in more than one accepted alliance/informal group, we used the expression "pro parte" ("p.p."). In contrast, we used the symbol "+" when we merged two clusters.

The diagnostic, constant and dominant species for each accepted cluster after TWINSPAN classification are shown in Appendix S3. For completeness, we also report the two clusters from Crimea and the Great Caucasus foothills in this Appendix.

\section{1 | Vegetation types}

We classified vegetation plots using the newly created classification expert system for the low-elevation Mediterranean pine forests. We also defined within the expert system the formulas for Crimean and Caucasian Pinus brutia forests. The expert system included 15 logical definitions of accepted alliances and other vegetation types. We applied this expert system to the non-resampled data set. The distribution of the plots classified as 12 accepted alliances and three informal groups is shown in Figure 4, along with the supposedly native distribution of the dominant pine species. Shortened lists of diagnostic species are shown in Table 2, while all the diagnostic, constant and dominant species for each alliance and the informal group of communities, based on the plots classified by the expert system, are listed in Appendix S4. Photos of typical stands of each alliance are provided in Figure 5. The alliances and informal groups are presented following the alphabetical order of the dominant pine species. Moreover, the floristic differences between Mediterranean thermophilous pine forests and evergreen oak forests are presented in Table 3. We include these forest in a new class and a previously described order:

Pinetea halepensis Bonari et M. Chytrý cl. nov.

Nomenclatural type (holotypus): Pinetalia halepensis Biondi, Blasi, Galdenzi, Pesaresi et Vagge in Biondi et al. 2014 (Biondi et al., 2014, p. 330)

Diagnostic species of the class: Pinus brutia, Pinus halepensis, Pinus pinaster, Pinus pinea.

Pinetalia halepensis Biondi, Blasi, Galdenzi, Pesaresi et Vagge in Biondi et al. 2014

Nomenclatural type: Pistacio lentisci-Pinion halepensis Biondi, Blasi, Galdenzi, Pesaresi et Vagge in Biondi et al. 2014

\subsection{1 | Thermo- to mesomediterranean Pinus brutia forests}

\section{Pinion brutiae Feinbrun 1959}

Acronym: Pin-Bru; Figures 4; 5a; 6; 7; Clusters $1+2$

Nomenclatural type (holotypus): Pinetum brutiae libanoticum Feinbrun 1959

Synonyms: Gonocytiso pterocladi-Pinion brutiae Barbéro, Chalabi, Nahal et Quézel ex Quézel et al. 1993 nom. inval. [ICPN Art. 2b]; Ptosimopappo-Quercion microphyllae Barbéro, Chalabi, Nahal et 


\section{Dominant pine:}

Pinus brutia

Pinus halepensis

Pinus pinaster

Pinus pinea
Thermomediterranean

Pinus brutia forests

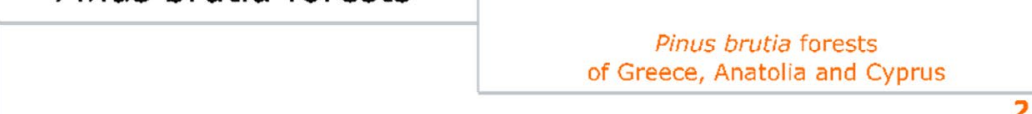

Mesomediterranean

Pinus brutia and

Pinus pinea forests

Pinus pinea forests of Anatolia and Lebanon

Pinus brutia forests

of Greece, E Mediterranean and Near East

Pinus halepensis forests

of the Mediterranean Basin

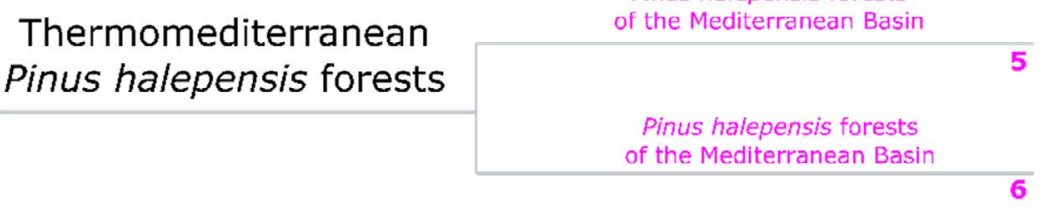

Pinus halepensis

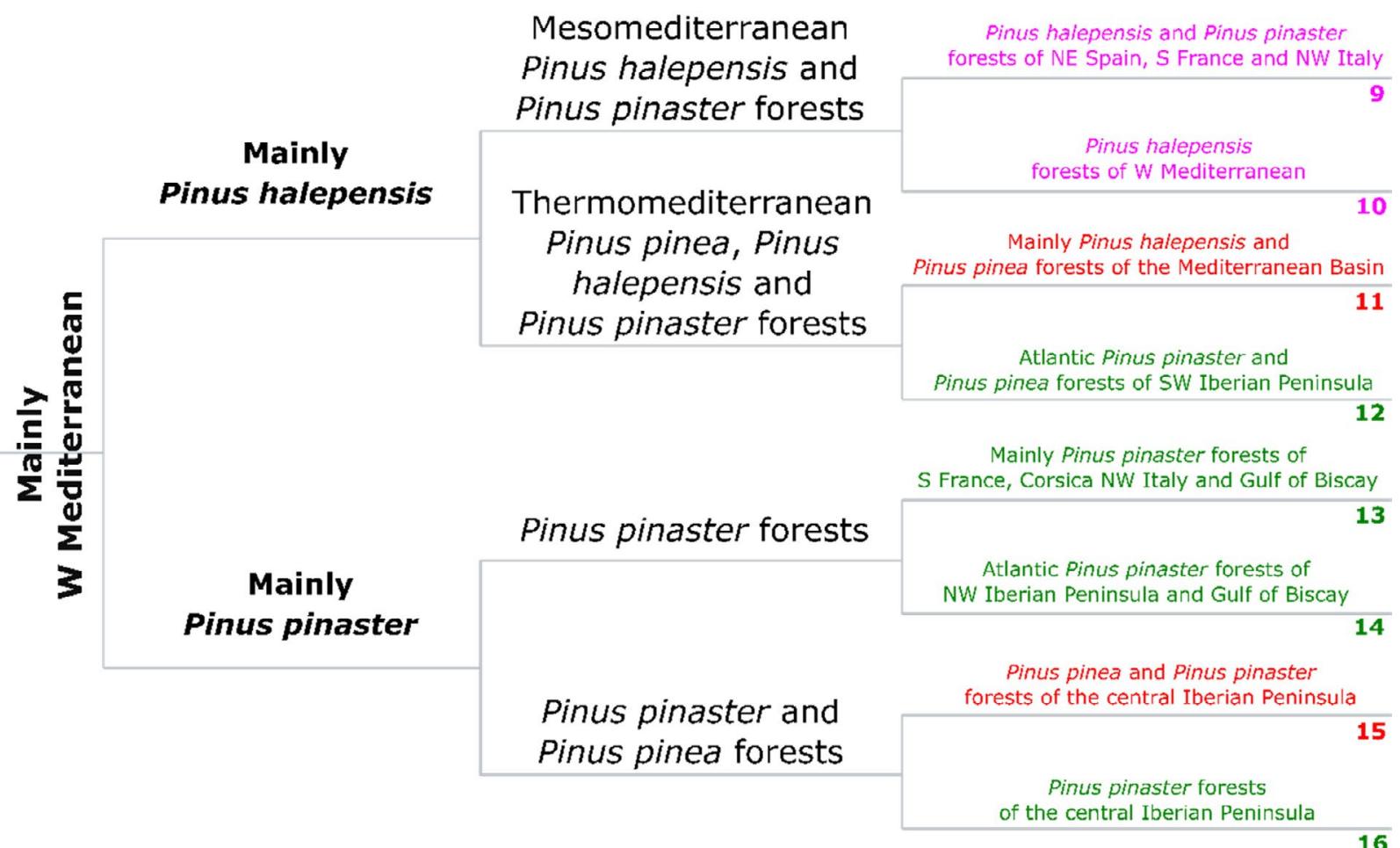

FIGURE 3 TWINSPAN dendrogram up to the fourth hierarchical level of division. In colour (right) the code of the TWINSPAN cluster (from 1 to 16 ) is given. The cluster approximately corresponding to Sarcopoterio spinosi-Pinion halepensis identified at a lower hierarchical level (i.e. within cluster 5) is not shown, as well as the Crimean and northwestern Caucasian clusters approximately corresponding to Jasmino fruticantis-Juniperion excelsae and Campanulo sibiricae-Pinion brutiae (within cluster 4)

Quézel ex Quézel et al. 1993 nom. inval. [ICPN Art. 2b]; Salvio fruticosae-Pinion brutiae Konstantinidis, Mucina et Bergmeier in Mucina et al. 2016 nom. inval. [ICPN Art. 5, 8].
Nomenclature comments: The invalid alliance names referred to in synonymy were proposed on the basis of geographical or lithological differences: calcareous or volcanic substrates in the 


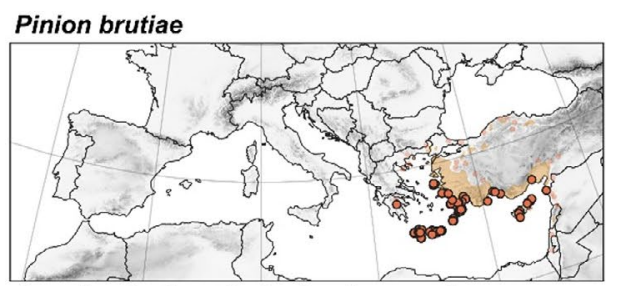

Jasmino fruticantis-Juniperion exce/sae

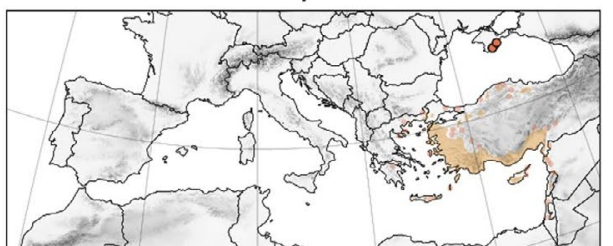

Thymo vulgaris-Pinion halepensis

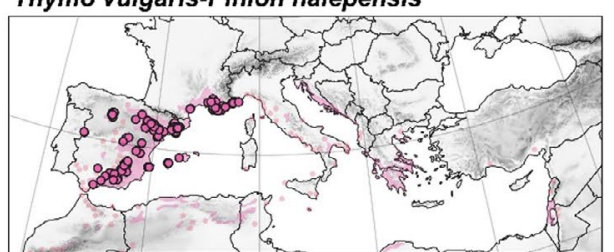

Pistacio lentisci-Pinion halepensis

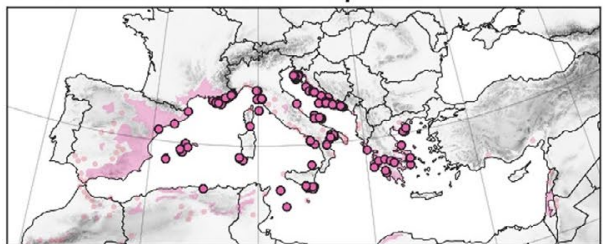

Coremato albi-Pinion pinastri

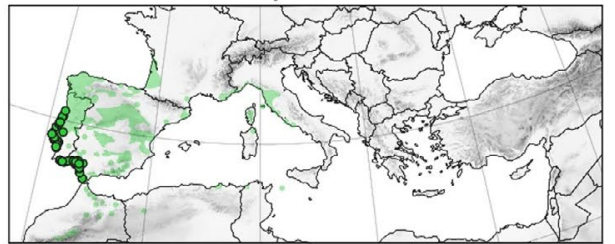

Lavandulo pedunculatae-Pinion pinastri

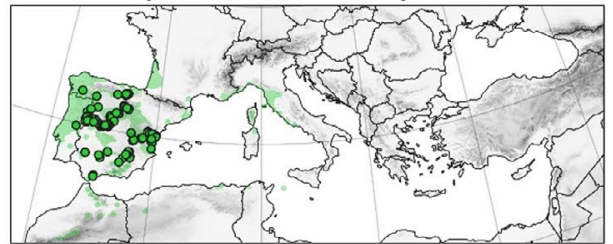

Central Iberian Pinus pinea forests

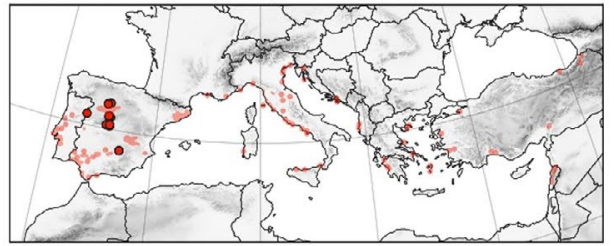

Pinion pineae

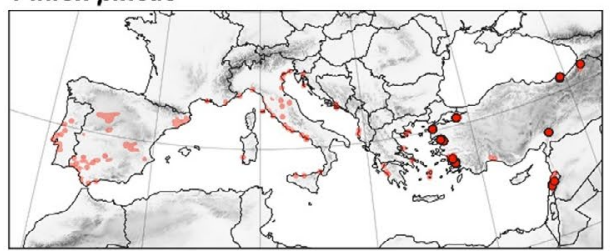

Styraco officinalis-Pinion brutiae

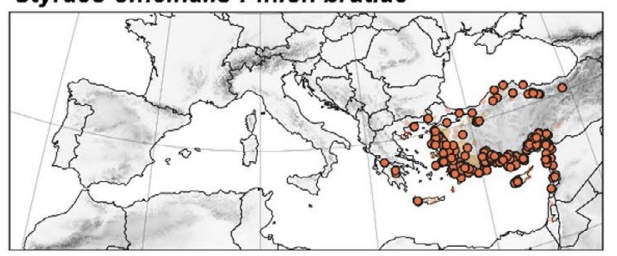

Campanulo sibiricae-Pinion brutiae

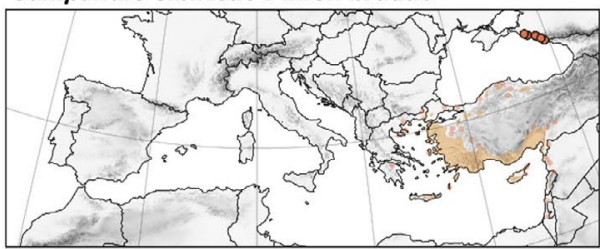

Rosmarino eriocalycis-Pinion halepensis

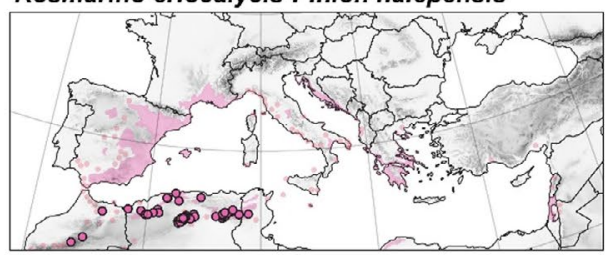

Sarcopoterio spinosi-Pinion halepensis

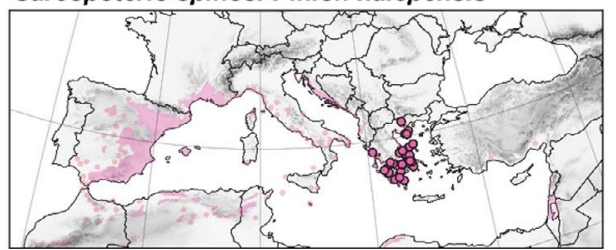

Atlantic Pinus pinaster forests

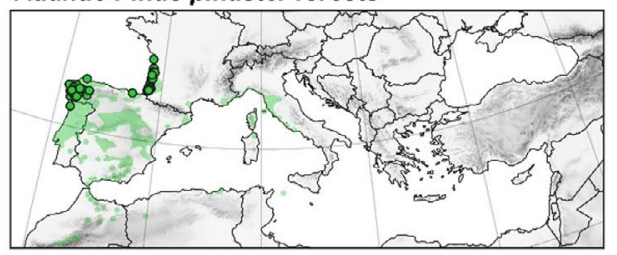

Genisto pilosae-Pinion pinastri

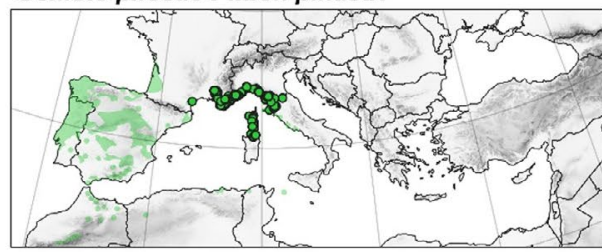

Mediterranean Pinus pinea forests

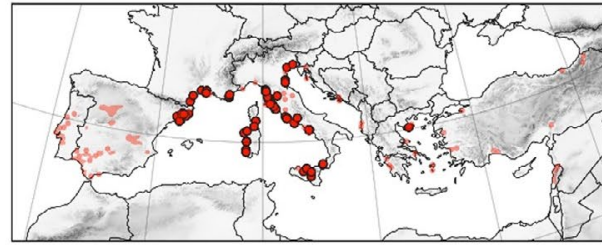

All plots

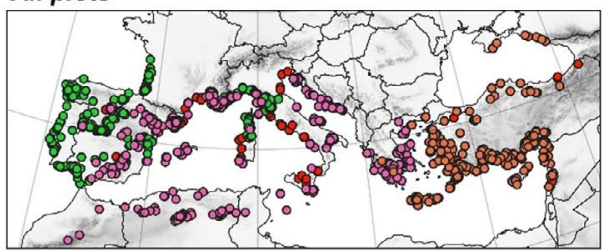

FIGURE 4 Distribution maps based on the plots assigned to alliances or informal groups of communities by the expert system ( $n=5,116)$. The shaded area represents the supposed native distribution of the dominant pine species (from Caudullo et al., 2017), while dots show the position of the classified vegetation plots (orange: Pinus brutia; violet: Pinus halepensis; green: Pinus pinaster; red: Pinus pinea) 
TAB LE 2 Shortened synoptic table of the percentage constancies of the diagnostic and most frequent species for the vegetation plots classified at the alliance level by the expert system and geographically resampled within each alliance; diagnostic species are sorted by decreasing values of the phi coefficient $(\Phi)$ for each alliance; only species with $\Phi>0.2$, Constancy Ratio (CR) $>1.5$ and $p<0.05$ (based on Fisher's exact test) are shown, indicated by grey shading; pines are shown at the top of the table, while non-diagnostic species with more than 300 occurrences across the whole table are reported at the bottom; the points represent species absence; see Appendix S4 for the full version of this table. See paragraphs 3.1.1-3.1.15 for alliance acronyms

\begin{tabular}{|c|c|c|c|c|c|c|c|c|c|c|c|c|c|c|c|}
\hline Alliance & $\begin{array}{l}\text { Pin- } \\
\text { Bru }\end{array}$ & $\begin{array}{l}\text { Sty- } \\
\text { Bru }\end{array}$ & $\begin{array}{l}\text { Jas- } \\
\text { Jun }\end{array}$ & $\begin{array}{l}\text { Cam- } \\
\text { Bru }\end{array}$ & $\begin{array}{l}\text { Thy- } \\
\text { Hal }\end{array}$ & $\begin{array}{l}\text { Ros- } \\
\text { Hal }\end{array}$ & $\begin{array}{l}\text { Pis- } \\
\text { Hal }\end{array}$ & $\begin{array}{l}\text { Sar- } \\
\text { Hal }\end{array}$ & $\begin{array}{l}\text { Cor- } \\
\text { Psr }\end{array}$ & $\begin{array}{l}\text { Atl- } \\
\text { Psr }\end{array}$ & $\begin{array}{l}\text { Lav- } \\
\text { Psr }\end{array}$ & $\begin{array}{l}\text { Gen- } \\
\text { Psr }\end{array}$ & $\begin{array}{l}\text { Cen- } \\
\text { Pna }\end{array}$ & $\begin{array}{l}\text { Med- } \\
\text { Pna }\end{array}$ & $\begin{array}{l}\text { Pin- } \\
\text { Pna }\end{array}$ \\
\hline No. of plots & 341 & 1,030 & 12 & 6 & 239 & 86 & 494 & 130 & 140 & 117 & 650 & 725 & 81 & 323 & 94 \\
\hline Pinus brutia & 100 & 100 & 100 & 100 & & . & . & . & . & . & . & . & . & . & 18 \\
\hline Pinus halepensis & . & 1 & . & . & 100 & 100 & 100 & 100 & 2 & . & 2 & 14 & . & 2 & . \\
\hline Pinus pinea & . & 1 & . & . & 4 & . & 1 & . & 89 & . & 1 & 1 & 100 & 100 & 100 \\
\hline
\end{tabular}

Pinion brutiae

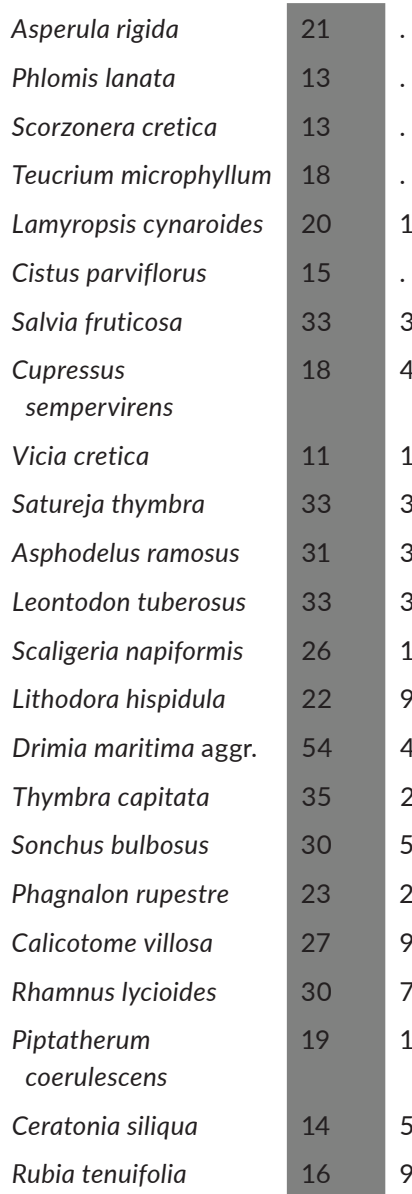

Styraco officinalis-Pinion brutiae

$\begin{array}{llll}\text { Eryngium falcatum } & 1 & 18 \\ \text { Quercus cerris } & \cdot & 16 & . \\ \text { Styrax officinalis } & 7 & 40 & . \\ \text { Daphne sericea } & 3 & 12 & . \\ \text { Quercus infectoria } & 1 & 30 & . \\ \text { Lathyrus aphaca } & 5 & 13 & . \\ \text { Crucianella latifolia } & 9 & 13 & .\end{array}$

.


TABLE 2 (Continued)

\begin{tabular}{|c|c|c|c|c|c|c|c|c|c|c|c|c|c|c|c|}
\hline Alliance & $\begin{array}{l}\text { Pin- } \\
\text { Bru }\end{array}$ & $\begin{array}{l}\text { Sty- } \\
\text { Bru }\end{array}$ & $\begin{array}{l}\text { Jas- } \\
\text { Jun }\end{array}$ & $\begin{array}{l}\text { Cam- } \\
\text { Bru }\end{array}$ & $\begin{array}{l}\text { Thy- } \\
\text { Hal }\end{array}$ & $\begin{array}{l}\text { Ros- } \\
\text { Hal }\end{array}$ & $\begin{array}{l}\text { Pis- } \\
\text { Hal }\end{array}$ & $\begin{array}{l}\text { Sar- } \\
\text { Hal }\end{array}$ & $\begin{array}{l}\text { Cor- } \\
\text { Psr }\end{array}$ & $\begin{array}{l}\text { Atl- } \\
\text { Psr }\end{array}$ & $\begin{array}{l}\text { Lav- } \\
\text { Psr }\end{array}$ & $\begin{array}{l}\text { Gen- } \\
\text { Psr }\end{array}$ & $\begin{array}{l}\text { Cen- } \\
\text { Pna }\end{array}$ & $\begin{array}{l}\text { Med- } \\
\text { Pna }\end{array}$ & $\begin{array}{l}\text { Pin- } \\
\text { Pna }\end{array}$ \\
\hline
\end{tabular}

Jasmino fruticantis-Juniperion excelsae

Jurinea ledebouri
Elymus nodosus
Linum austriacum
Thymus roegneri

Asparagus verticillatus

Odontarrhena tortuosa

Pistacia atlantica

Bromopsis cappadocica

Galatella villosa

Seseli dichotomum

Veronica multifida

Centaurea diffusa

Fumana procumbens

Inula ensifolia

Juniperus excelsa

Melica ciliata

Fumana arabica $\quad 12 \quad 6$

Poa sterilis

Jasminum fruticans 110

Linum strictum

aggr. (incl.

L. corymbulosum)

Achnatherum

$16 \quad 27$

bromoides (incl.

A. fallacinum)

Teucrium polium aggr. 620

Teucrium chamaedrys 118

Galium biebersteinii

Bothriochloa

ischaemum

Carex flacca

Carex halleriana

Salvia officinalis

Ruscus aculeatus

Rhus coriaria

Convolvulus cantabrica

$7 \quad 14$

82

. 9

Campanulo sibiricae-Pinion brutiae

Sesleria alba

Echinops

sphaerocephalus

Astragalus cicer

Hedera colchica

Argyrolobium

biebersteinii

Smilax excelsa

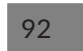

92

67

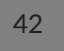

42
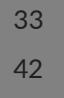

33
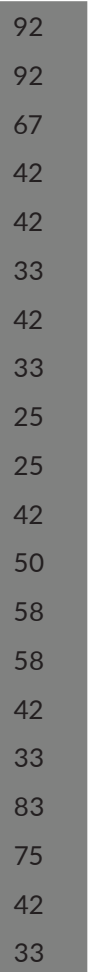

100

17

1

17

33

22

$5 \quad 6 \quad 25$

3

25

58

50

17

$42 \quad 33$

$17 \quad 17$

$17 \quad 17$
17
$33 \quad 17$

$17 \quad 29$

10

33

17
13

1$$
29
$$

19

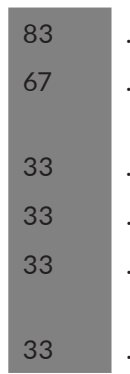

27 
TABLE 2 (Continued)

\begin{tabular}{|c|c|c|c|c|c|c|c|c|c|c|c|c|c|c|c|}
\hline Alliance & $\begin{array}{l}\text { Pin- } \\
\text { Bru }\end{array}$ & $\begin{array}{l}\text { Sty- } \\
\text { Bru }\end{array}$ & $\begin{array}{l}\text { Jas- } \\
\text { Jun }\end{array}$ & $\begin{array}{l}\text { Cam- } \\
\text { Bru }\end{array}$ & $\begin{array}{l}\text { Thy- } \\
\text { Hal }\end{array}$ & $\begin{array}{l}\text { Ros- } \\
\text { Hal }\end{array}$ & $\begin{array}{l}\text { Pis- } \\
\text { Hal }\end{array}$ & $\begin{array}{l}\text { Sar- } \\
\text { Hal }\end{array}$ & $\begin{array}{l}\text { Cor- } \\
\text { Psr }\end{array}$ & $\begin{array}{l}\text { Atl- } \\
\text { Psr }\end{array}$ & $\begin{array}{l}\text { Lav- } \\
\text { Psr }\end{array}$ & $\begin{array}{l}\text { Gen- } \\
\text { Psr }\end{array}$ & $\begin{array}{l}\text { Cen- } \\
\text { Pna }\end{array}$ & $\begin{array}{l}\text { Med- } \\
\text { Pna }\end{array}$ & $\begin{array}{l}\text { Pin- } \\
\text { Pna }\end{array}$ \\
\hline Paeonia mascula & . & 1 & . & 33 & . & . & . & . & . & . & . & . & . & . & . \\
\hline Lonicera caprifolium & . & 1 & . & 33 & . & . & 3 & . & . & . & . & . & . & 2 & . \\
\hline $\begin{array}{l}\text { Physospermum } \\
\text { cornubiense }\end{array}$ & . & 1 & . & 33 & . & . & 1 & . & . & . & 4 & 3 & . & . & . \\
\hline $\begin{array}{l}\text { Epipactis helleborine } \\
\text { aggr. }\end{array}$ & . & 3 & . & 33 & 1 & 2 & . & . & . & . & 2 & 5 & . & 1 & . \\
\hline $\begin{array}{l}\text { Brachypodium } \\
\text { pinnatum }\end{array}$ & . & 4 & . & 50 & . & . & 2 & . & . & . & 1 & 8 & . & 1 & 4 \\
\hline Viola alba & . & 1 & . & 67 & 1 & . & 1 & 1 & . & . & 1 & 14 & . & 4 & . \\
\hline Bituminaria bituminosa & 7 & 6 & . & 67 & 15 & . & 4 & 2 & . & . & 6 & 11 & 6 & 3 & 14 \\
\hline Coronilla coronata & . & . & 8 & 33 & . & . & . & . & . & . & . & . & . & . & . \\
\hline Stachys recta & . & . & 8 & 33 & . & . & 2 & 3 & . & . & . & 10 & . & 1 & . \\
\hline Clematis vitalba & . & 1 & . & 33 & 1 & . & 1 & . & . & . & 1 & 10 & . & 4 & 4 \\
\hline $\begin{array}{l}\text { Brachypodium } \\
\text { sylvaticum }\end{array}$ & 1 & 9 & . & 50 & . & . & 2 & 8 & . & 1 & 12 & 6 & . & 22 & . \\
\hline Hedera helix & 1 & 3 & . & 67 & . & . & 9 & . & . & 29 & 2 & 20 & . & 31 & . \\
\hline
\end{tabular}

Thymo vulgaris-Pinion halepensis

\begin{tabular}{|c|c|}
\hline Stipa juncea & . \\
\hline Thymelaea tinctoria & . \\
\hline Centaurea linifolia & . \\
\hline $\begin{array}{l}\text { Helianthemum } \\
\text { marifolium }\end{array}$ & . \\
\hline Bupleurum fruticescens & . \\
\hline Fumana ericoides & . \\
\hline Globularia vulgaris & . \\
\hline Genista scorpius & . \\
\hline Lavandula latifolia & . \\
\hline Staehelina dubia & . \\
\hline Koeleria vallesiana & . \\
\hline Bupleurum rigidum & . \\
\hline Coris monspeliensis & . \\
\hline Festuca ovina aggr. & . \\
\hline Argyrolobium zanonii & . \\
\hline Helictochloa bromoides & . \\
\hline Erica multiflora & . \\
\hline Polygala rupestris & . \\
\hline Helichrysum stoechas & 19 \\
\hline Cistus albidus & \\
\hline
\end{tabular}

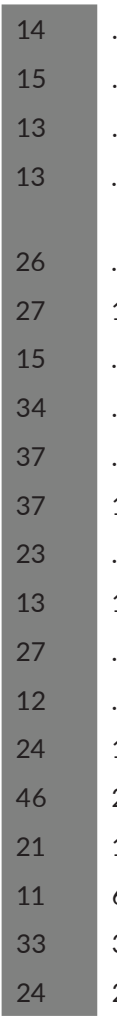

(Continues) 
TABLE 2 (Continued)

\begin{tabular}{|c|c|c|c|c|c|c|c|c|c|c|c|c|c|c|c|}
\hline Alliance & $\begin{array}{l}\text { Pin- } \\
\text { Bru }\end{array}$ & $\begin{array}{l}\text { Sty- } \\
\text { Bru }\end{array}$ & $\begin{array}{l}\text { Jas- } \\
\text { Jun }\end{array}$ & $\begin{array}{l}\text { Cam- } \\
\text { Bru }\end{array}$ & $\begin{array}{l}\text { Thy- } \\
\text { Hal }\end{array}$ & $\begin{array}{l}\text { Ros- } \\
\text { Hal }\end{array}$ & $\begin{array}{l}\text { Pis- } \\
\text { Hal }\end{array}$ & $\begin{array}{l}\text { Sar- } \\
\text { Hal }\end{array}$ & $\begin{array}{l}\text { Cor- } \\
\text { Psr }\end{array}$ & $\begin{array}{l}\text { Atl- } \\
\text { Psr }\end{array}$ & $\begin{array}{l}\text { Lav- } \\
\text { Psr }\end{array}$ & $\begin{array}{l}\text { Gen- } \\
\text { Psr }\end{array}$ & $\begin{array}{l}\text { Cen- } \\
\text { Pna }\end{array}$ & $\begin{array}{l}\text { Med- } \\
\text { Pna }\end{array}$ & $\begin{array}{l}\text { Pin- } \\
\text { Pna }\end{array}$ \\
\hline $\begin{array}{l}\text { Linum suffruticosum } \\
\text { aggr. }\end{array}$ & . & . & . & . & 24 & 5 & . & . & . & . & 2 & 15 & 1 & 1 & . \\
\hline Ononis minutissima & . & . & . & . & 20 & . & 1 & . & . & . & . & 13 & . & 1 & . \\
\hline
\end{tabular}

Rosmarino eriocalycis-Pinion halepensis

\begin{tabular}{|c|c|}
\hline Rosmarinus eriocalyx & . \\
\hline Thymus munbyanus & . \\
\hline Centaurea boissieri & . \\
\hline $\begin{array}{l}\text { Helianthemum } \\
\text { virgatum }\end{array}$ & . \\
\hline Odontarrhena alpestris & . \\
\hline Bombycilaena discolor & . \\
\hline Bupleurum atlanticum & . \\
\hline Ebenus pinnata & . \\
\hline Catapodium marinum & . \\
\hline Eruca vesicaria & . \\
\hline Arabis nova & . \\
\hline Alyssum granatense & . \\
\hline $\begin{array}{l}\text { Macrochloa } \\
\text { tenacissima }\end{array}$ & . \\
\hline Anisantha rubens & 1 \\
\hline Hornungia petraea & . \\
\hline $\begin{array}{l}\text { Teucrium } \\
\text { pseudochamaepitys }\end{array}$ & . \\
\hline Cistus clusii & . \\
\hline Filago pyramidata & 1 \\
\hline $\begin{array}{l}\text { Helianthemum } \\
\text { cinereum }\end{array}$ & . \\
\hline Globularia alypum & 2 \\
\hline Paronychia argentea & . \\
\hline $\begin{array}{c}\text { Ampelodesmos } \\
\text { mauritanicus }\end{array}$ & . \\
\hline
\end{tabular}

\section{Pistacio lentisci-Pinion halepensis}

$\begin{array}{lll}\text { Teucrium fruticans } & \cdot & \cdot \\ \text { Viburnum tinus } & \cdot & \cdot \\ \text { Allium subhirsutum } & 4 & \cdot \\ \text { Myrtus communis } & 10 & 19 \\ \text { Cistus monspeliensis } & \cdot & \cdot \\ \text { Lonicera implexa } & \cdot & 1 \\ \text { Asparagus acutifolius } & 15 & 29 \\ \text { Smilax aspera } & 17 & 21\end{array}$

Sarcopoterio spinosi-Pinion halepensis

$\begin{array}{lll}\text { Cyclamen graecum } & 1 & . \\ \text { Helictotrichon } & 1 & 1 \\ \text { convolutum } & & \\ \text { Phlomis fruticosa } & 4 & 3 \\ \text { Luzula nodulosa } & 5 & 3\end{array}$
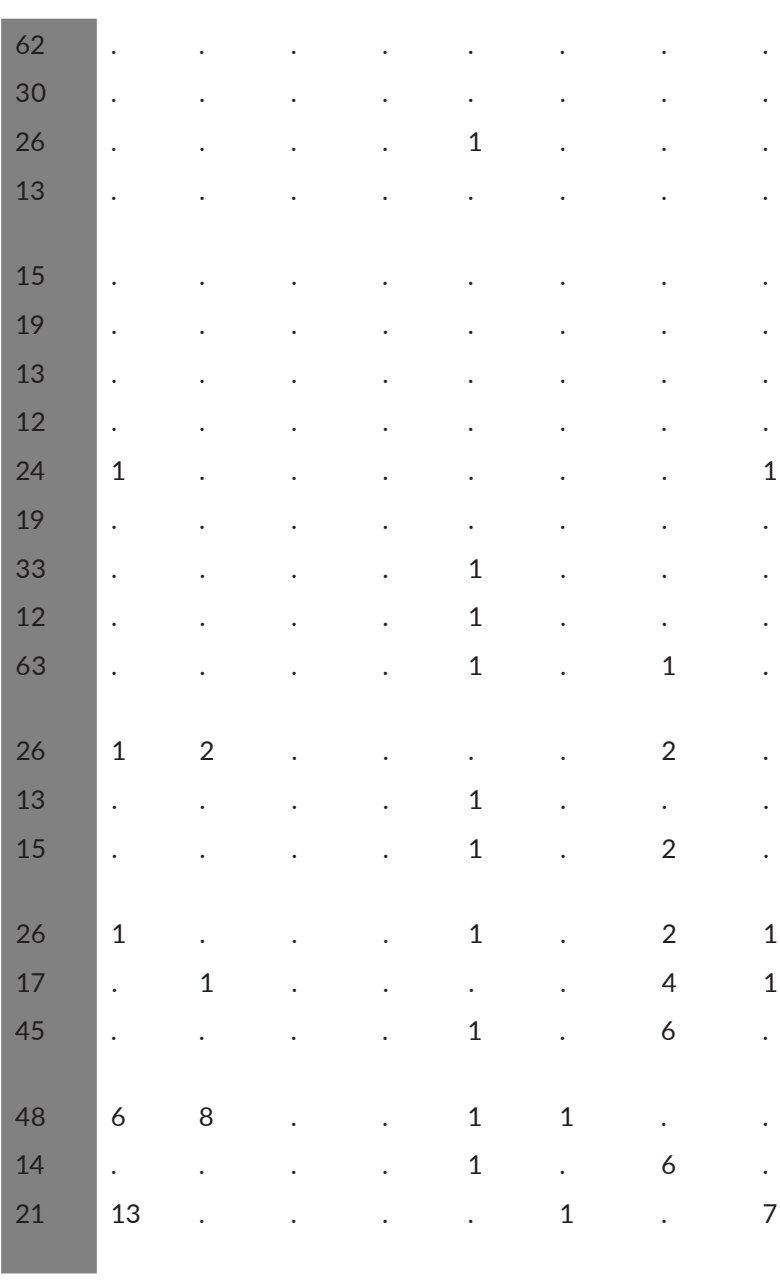

$\begin{array}{lllllllll}11 & . & 1 & . & 1 & . & . & 1 & . \\ 21 & 1 & . & . & 4 & 2 & . & 5 & . \\ 15 & 2 & . & . & . & 1 & . & 4 & . \\ 52 & 1 & 15 & . & 1 & 19 & . & 19 & 1 \\ 28 & 4 & 4 & . & 1 & 7 & . & 14 & . \\ 39 & 17 & 1 & . & 2 & 20 & . & 16 & . \\ 72 & 27 & 20 & . & 4 & 10 & 30 & 46 & 28 \\ 61 & 25 & 14 & . & 1 & 27 & . & 39 & 9\end{array}$

(Continues) 
TABLE 2 (Continued)

\begin{tabular}{|c|c|c|c|c|c|c|c|c|c|c|c|c|c|c|c|}
\hline Alliance & $\begin{array}{l}\text { Pin- } \\
\text { Bru }\end{array}$ & $\begin{array}{l}\text { Sty- } \\
\text { Bru }\end{array}$ & $\begin{array}{l}\text { Jas- } \\
\text { Jun }\end{array}$ & $\begin{array}{l}\text { Cam- } \\
\text { Bru }\end{array}$ & $\begin{array}{l}\text { Thy- } \\
\text { Hal }\end{array}$ & $\begin{array}{l}\text { Ros- } \\
\text { Hal }\end{array}$ & $\begin{array}{l}\text { Pis- } \\
\text { Hal }\end{array}$ & $\begin{array}{l}\text { Sar- } \\
\mathrm{Hal}\end{array}$ & $\begin{array}{l}\text { Cor- } \\
\text { Psr }\end{array}$ & $\begin{array}{l}\text { Atl- } \\
\text { Psr }\end{array}$ & $\begin{array}{l}\text { Lav- } \\
\text { Psr }\end{array}$ & $\begin{array}{l}\text { Gen- } \\
\text { Psr }\end{array}$ & $\begin{array}{l}\text { Cen- } \\
\text { Pna }\end{array}$ & $\begin{array}{l}\text { Med- } \\
\text { Pna }\end{array}$ & $\begin{array}{l}\text { Pin- } \\
\text { Pna }\end{array}$ \\
\hline Anthyllis hermanniae & 13 & 1 & . & . & . & . & 2 & 45 & . & . & . & 1 & . & . & 13 \\
\hline $\begin{array}{l}\text { Hypochaeris } \\
\text { achyrophorus }\end{array}$ & 3 & 1 & . & . & . & . & 5 & 20 & . & . & . & 2 & . & 4 & 12 \\
\hline Carex distachya & 5 & 3 & . & . & . & . & 9 & 18 & 1 & . & 3 & 2 & 1 & 14 & 1 \\
\hline Aira elegantissima & 4 & 3 & . & . & 1 & . & 1 & 15 & . & . & . & 1 & . & . & 14 \\
\hline
\end{tabular}

Coremato albi-Pinion pinastri

Cistus halimifolius

Cistus calycinus

Ulex genistoides

Corema album

Cytisus grandiflorus

Aristolochia baetica

Cistus crispus

Osyris lanceolata

Carpobrotus edulis

Helichrysum italicum

Chamaerops humilis

Cistus libanotis

Ulex parviflorus

Genista triacanthos

Cistus salviifolius

Lavandula stoechas

Juniperus phoenicea

Lagurus ovatus

Atlantic Pinus pinaster forests

Ulex minor
Pseudarrhenatherum
longifolium
Daboecia cantabrica
Melampyrum pratense
Agrostis curtisii
Erica cinerea
Pedicularis sylvatica
Glandora diffusa
Quercus robur
Ulex europaeus
Digitalis purpurea
Potentilla erecta
Frangula alnus
Lonicera periclymenum
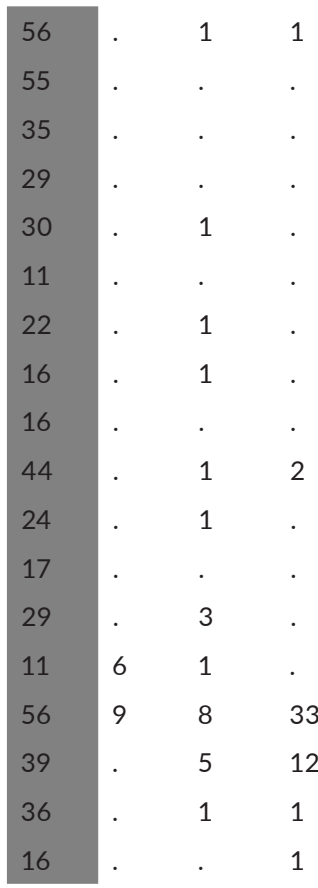

1

2$$
1
$$$$
2
$$$$
1-25
$$$$
23
$$$$
10
$$$$
3 \text {. }
$$

$$
8
$$$$
14
$$$$
31
$$$$
32
$$

$$
\begin{array}{ccc}
22 & 27 & 4 \\
& 8 & 3
\end{array}
$$


TABLE 2 (Continued)

\begin{tabular}{|c|c|c|c|c|c|c|c|c|c|c|c|c|c|c|c|}
\hline Alliance & $\begin{array}{l}\text { Pin- } \\
\text { Bru }\end{array}$ & $\begin{array}{l}\text { Sty- } \\
\text { Bru }\end{array}$ & $\begin{array}{l}\text { Jas- } \\
\text { Jun }\end{array}$ & $\begin{array}{l}\text { Cam- } \\
\text { Bru }\end{array}$ & $\begin{array}{l}\text { Thy- } \\
\text { Hal }\end{array}$ & $\begin{array}{l}\text { Ros- } \\
\text { Hal }\end{array}$ & $\begin{array}{l}\text { Pis- } \\
\text { Hal }\end{array}$ & $\begin{array}{l}\text { Sar- } \\
\text { Hal }\end{array}$ & $\begin{array}{l}\text { Cor- } \\
\text { Psr }\end{array}$ & $\begin{array}{l}\text { Atl- } \\
\text { Psr }\end{array}$ & $\begin{array}{l}\text { Lav- } \\
\text { Psr }\end{array}$ & $\begin{array}{l}\text { Gen- } \\
\text { Psr }\end{array}$ & $\begin{array}{l}\text { Cen- } \\
\text { Pna }\end{array}$ & $\begin{array}{l}\text { Med- } \\
\text { Pna }\end{array}$ & $\begin{array}{l}\text { Pin- } \\
\text { Pna }\end{array}$ \\
\hline Ilex aquifolium & . & 1 & . & . & . & . & . & . & . & 19 & 1 & 4 & . & . & . \\
\hline Glandora prostrata & . & . & . & . & . & . & . & . & 5 & 18 & 2 & . & . & . & . \\
\hline Erica umbellata & . & . & . & . & 1 & . & . & . & 3 & 32 & 10 & . & . & . & . \\
\hline Molinia caerulea aggr. & . & . & . & . & . & . & . & . & . & 20 & . & 8 & . & 1 & . \\
\hline $\begin{array}{c}\text { Corynephorus } \\
\text { canescens }\end{array}$ & . & . & . & . & . & . & . & . & 8 & 20 & 7 & 1 & 9 & . & . \\
\hline Simethis planifolia & . & . & . & . & 1 & . & 2 & . & 1 & 22 & 10 & 1 & . & 1 & . \\
\hline Quercus suber & . & . & . & . & 2 & . & 3 & . & 4 & 33 & 3 & 16 & . & 10 & . \\
\hline
\end{tabular}

Lavandulo pedunculatae-Pinion pinastri

\begin{tabular}{|c|c|c|c|c|c|c|c|c|c|c|c|c|c|c|}
\hline Cistus populifolius & . & . & ${ }^{\circ}$ & . & . & . & . & . & . & . & 14 & & . & . \\
\hline Festuca elegans & . & . & . & . & . & . & . & . & . & . & 12 & & . & . \\
\hline Erica australis & . & . & . & . & 1 & . & . & . & 1 & . & 29 & & . & . \\
\hline Digitalis thapsi & . & . & . & . & . & . & . & . & . & . & 23 & & 1 & . \\
\hline $\begin{array}{l}\text { Aristolochia } \\
\text { paucinervis }\end{array}$ & . & . & . & . & . & . & . & . & . & . & 13 & • & 1 & . \\
\hline Genista tridentata & . & . & . & . & 1 & . & . & . & 2 & 2 & 22 & $\cdot$ & . & . \\
\hline Cytisus multiflorus & . & . & . & . & . & . & . & . & . & 2 & 16 & & 1 & . \\
\hline Quercus pyrenaica & . & . & . & . & . & . & . & . & . & 8 & 36 & & . & . \\
\hline $\begin{array}{l}\text { Anarrhinum } \\
\text { bellidifolium }\end{array}$ & . & . & . & . & 1 & . & . & . & . & 4 & 14 & . & . & . \\
\hline Hypochaeris radicata & . & . & . & . & 3 & . & 1 & . & . & 7 & 37 & 1 & 12 & 4 \\
\hline Tuberaria lignosa & . & . & . & . & 2 & . & 3 & . & . & 3 & 11 & 4 & . & 1 \\
\hline Arrhenatherum elatius & . & 1 & . & . & 6 & . & . & . & 1 & . & 21 & 3 & 7 & 2 \\
\hline Holcus lanatus & . & . & . & . & . & . & 1 & . & . & 1 & 13 & 5 & . & 5 \\
\hline Cytisus striatus & . & . & . & . & . & . & . & . & . & 8 & 19 & & . & . \\
\hline $\begin{array}{l}\text { Agrostis } \\
\text { castellana }+ \text { tenuis }\end{array}$ & . & . & . & 17 & . & . & . & . & 1 & 8 & 38 & 6 & 15 & 5 \\
\hline Clinopodium vulgare & . & 8 & . & . & . & . & . & 2 & . & . & 24 & 3 & . & . \\
\hline Centaurea alba aggr. & . & . & . & . & . & . & . & . & . & . & 20 & . & 11 & 1 \\
\hline Cistus umbellatus & . & . & . & . & . & . & . & . & 1 & . & 17 & . & 10 & . \\
\hline Micropyrum tenellum & . & . & . & . & . & . & . & . & . & . & 16 & . & 10 & . \\
\hline Cistus psilosepalus & . & . & . & . & . & . & . & . & 1 & 12 & 18 & & . & . \\
\hline Filago minima & . & . & . & . & . & . & . & . & 1 & . & 16 & . & 11 & . \\
\hline Cytisus scoparius & . & . & . & . & 1 & . & . & . & . & 19 & 24 & 3 & 12 & 3 \\
\hline
\end{tabular}

\section{Genisto pilosae-Pinion pinastri}

\begin{tabular}{|c|c|c|c|c|c|c|c|c|c|c|c|c|}
\hline Knautia purpurea & . & . & . & . & 1 & . & . & . & . & . & . & 20 \\
\hline Sesleria autumnalis & . & . & . & . & 1 & $\cdot$ & 1 & . & . & . & . & 14 \\
\hline Rosa agrestis & ${ }^{\circ}$ & . & . & . & 1 & . & . & . & . & . & 1 & 12 \\
\hline $\begin{array}{l}\text { Cytisophyllum } \\
\text { sessilifolium }\end{array}$ & . & . & . & . & 1 & . & . & . & . & . & . & 12 \\
\hline $\begin{array}{l}\text { Polygala nicaeensis } \\
\text { aggr. }\end{array}$ & . & . & . & . & 1 & . & . & 2 & . & . & . & 13 \\
\hline Prunella hyssopifolia & . & . & . & . & 2 & . & . & . & . & $\cdot$ & . & 14 \\
\hline Teucrium montanum & $\cdot$ & . & . & . & 4 & . & . & . & . & . & . & 26 \\
\hline Centaurea jacea & . & . & . & . & 2 & . & . & . & . & . & 1 & 11 \\
\hline Sorbus domestica & . & . & . & . & 1 & . & 1 & . & . & . & 1 & 14 \\
\hline
\end{tabular}


TABLE 2 (Continued)

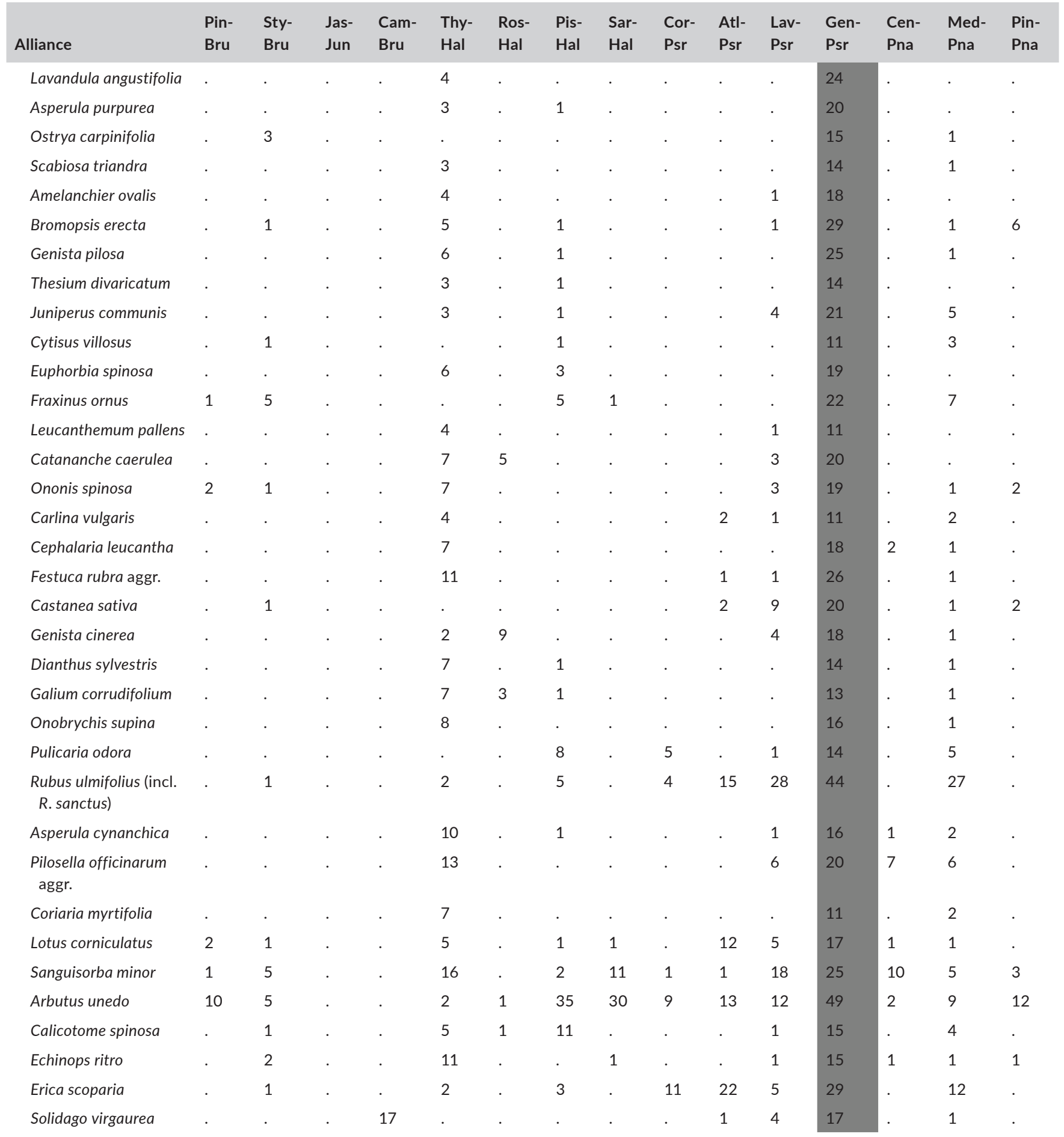

\section{Central Iberian Pinus pinea forests}

$\begin{array}{lcc}\text { Calendula arvensis } & \cdot & 1 \\ \text { Asphodelus serotinus } & \cdot & . \\ \text { Silene nocturna } & 1 & . \\ \text { Plantago lagopus } & 1 & 1 \\ \text { Daucus durieua } & \cdot & . \\ \text { Vicia disperma } & \cdot & 1\end{array}$

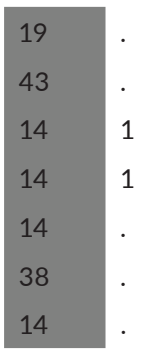

(Continues) 
TABLE 2 (Continued)

\begin{tabular}{|c|c|c|c|c|c|c|c|c|c|c|c|c|c|c|c|}
\hline Alliance & $\begin{array}{l}\text { Pin- } \\
\text { Bru }\end{array}$ & $\begin{array}{l}\text { Sty- } \\
\text { Bru }\end{array}$ & $\begin{array}{l}\text { Jas- } \\
\text { Jun }\end{array}$ & $\begin{array}{l}\text { Cam- } \\
\text { Bru }\end{array}$ & $\begin{array}{l}\text { Thy- } \\
\text { Hal }\end{array}$ & $\begin{array}{l}\text { Ros- } \\
\text { Hal }\end{array}$ & $\begin{array}{l}\text { Pis- } \\
\text { Hal }\end{array}$ & $\begin{array}{l}\text { Sar- } \\
\text { Hal }\end{array}$ & $\begin{array}{l}\text { Cor- } \\
\text { Psr }\end{array}$ & $\begin{array}{l}\text { Atl- } \\
\text { Psr }\end{array}$ & $\begin{array}{l}\text { Lav- } \\
\text { Psr }\end{array}$ & $\begin{array}{l}\text { Gen- } \\
\text { Psr }\end{array}$ & $\begin{array}{l}\text { Cen- } \\
\text { Pna }\end{array}$ & $\begin{array}{l}\text { Med- } \\
\text { Pna }\end{array}$ & $\begin{array}{l}\text { Pin- } \\
\text { Pna }\end{array}$ \\
\hline Anisantha diandra & 1 & 1 & . & . & . & . & . & . & 1 & . & 1 & . & 30 & 2 & . \\
\hline Echium plantagineum & . & 1 & . & . & . & . & 1 & . & . & . & 1 & . & 14 & 1 & . \\
\hline Silene gallica & . & 1 & . & . & . & . & 1 & . & . & . & 1 & 1 & 11 & 1 & . \\
\hline $\begin{array}{l}\text { Carduus } \\
\text { pycnocephalus }\end{array}$ & 1 & 1 & . & . & . & . & 1 & 1 & . & . & . & 1 & 25 & 1 & 2 \\
\hline Leontodon saxatilis & . & . & . & . & 1 & . & 1 & . & . & . & 4 & . & 48 & 1 & . \\
\hline Centaurea aristata & . & . & . & . & . & . & . & . & . & . & 2 & . & 21 & . & . \\
\hline Anthemis arvensis & . & 1 & . & . & . & . & 1 & 1 & . & . & 4 & 1 & 32 & 1 & . \\
\hline Jasione sessiliflora & . & . & . & . & . & . & . & . & . & . & 1 & . & 11 & . & . \\
\hline Crepis vesicaria & 1 & . & . & . & 6 & . & 6 & . & . & . & 2 & 1 & 47 & 3 & . \\
\hline Viola kitaibeliana & . & . & . & . & 1 & . & . & . & . & . & 2 & . & 14 & . & . \\
\hline Mibora minima & . & . & . & . & . & . & . & . & . & 2 & 1 & . & 12 & . & . \\
\hline Arrhenatherum album & . & . & . & . & . & . & . & . & 5 & . & 1 & . & 32 & . & . \\
\hline Retama sphaerocarpa & . & . & . & . & 2 & 1 & 1 & . & . & . & 5 & . & 26 & . & . \\
\hline Hypochaeris glabra & . & . & . & . & . & . & 1 & . & 7 & 5 & 5 & 1 & 32 & 3 & 1 \\
\hline Silene scabriflora & . & . & . & . & . & . & . & . & 1 & . & 3 & . & 11 & . & . \\
\hline Tragopogon porrifolius & 2 & 2 & . & . & . & . & . & 1 & . & . & 1 & 1 & 12 & . & 3 \\
\hline $\begin{array}{c}\text { Centranthus } \\
\text { calcitrapae }\end{array}$ & 3 & . & . & . & 1 & . & 1 & 1 & 4 & . & 4 & . & 15 & 1 & . \\
\hline Lupinus angustifolius & . & . & . & . & . & . & . & . & . & . & 4 & . & 12 & . & 1 \\
\hline Anisantha madritensis & 1 & 1 & . & . & 1 & . & 2 & 3 & 1 & . & 1 & . & 12 & 4 & . \\
\hline $\begin{array}{l}\text { Asterolinon } \\
\text { linum-stellatum }\end{array}$ & 6 & 1 & . & . & 2 & 12 & 4 & 3 & 9 & 1 & 3 & . & 38 & 3 & 1 \\
\hline Vicia lathyroides & 2 & 1 & . & . & . & . & . & . & . & . & 4 & 1 & 17 & . & 5 \\
\hline Sanguisorba verrucosa & . & 1 & . & . & 4 & . & . & . & . & . & 17 & 1 & 52 & . & . \\
\hline Urospermum picroides & 8 & 3 & . & . & . & . & 2 & 2 & 1 & . & 1 & . & 25 & 1 & 3 \\
\hline Crepis capillaris & . & . & . & . & . & . & . & . & 1 & 1 & 5 & . & 15 & . & . \\
\hline Lathyrus angulatus & . & . & . & . & . & . & . & . & . & . & 4 & . & 11 & . & . \\
\hline Trachynia distachya & 8 & 4 & . & . & 2 & 7 & 4 & 14 & 1 & . & 3 & 1 & 37 & 7 & 2 \\
\hline Aira caryophyllea & . & 1 & . & . & . & . & 2 & . & 1 & . & 9 & 1 & 23 & 7 & 4 \\
\hline Campanula rapunculus & . & 1 & . & . & 1 & . & 1 & . & . & . & 4 & 6 & 15 & 1 & . \\
\hline Linaria spartea & . & . & . & . & . & . & . & . & 4 & . & 4 & . & 11 & . & . \\
\hline Senecio lividus & . & . & . & . & . & . & . & . & 1 & 6 & 2 & 5 & 14 & 1 & . \\
\hline Vulpia myuros & . & 1 & . & . & . & . & 1 & . & 1 & 2 & 12 & 1 & 26 & 1 & 4 \\
\hline Thapsia villosa & . & . & . & . & 2 & . & . & . & 25 & . & 16 & 1 & 53 & 2 & . \\
\hline Avena barbata & 3 & 3 & . & . & . & . & 2 & 5 & 1 & . & 4 & 1 & 42 & 5 & 20 \\
\hline Leopoldia comosa & 6 & 4 & . & . & . & . & 2 & 8 & . & . & 1 & . & 17 & 2 & 9 \\
\hline Umbilicus rupestris & . & 1 & . & . & . & . & . & . & . & 2 & 6 & 3 & 11 & 1 & 1 \\
\hline Anisantha tectorum & $\cdot$ & 1 & 8 & . & . & . & . & . & . & . & 3 & . & 16 & . & 6 \\
\hline Carlina corymbosa & 10 & 1 & . & . & 8 & 1 & 1 & 2 & 6 & . & 23 & 6 & 43 & 1 & 22 \\
\hline Briza maxima & 9 & 3 & . & . & 1 & . & 11 & 2 & 28 & . & 25 & 4 & 53 & 13 & 28 \\
\hline Tuberaria guttata & 1 & 1 & . & . & 1 & 1 & 1 & . & 16 & 2 & 21 & 2 & 38 & 5 & 13 \\
\hline Coronilla scorpioides & 4 & 3 & 17 & 17 & 1 & 3 & 7 & 1 & . & . & . & . & 27 & 1 & 2 \\
\hline Anthyllis lotoides & . & . & . & . & . & . & . & . & . & . & 11 & . & 16 & & . \\
\hline Teesdalia coronopifolia & . & . & . & . & . & . & . & . & . & . & 8 & 1 & 11 & & . \\
\hline
\end{tabular}


TABLE 2 (Continued)

\begin{tabular}{|c|c|c|c|c|c|c|c|c|c|c|c|c|c|c|c|}
\hline Alliance & $\begin{array}{l}\text { Pin- } \\
\text { Bru }\end{array}$ & $\begin{array}{l}\text { Sty- } \\
\text { Bru }\end{array}$ & $\begin{array}{l}\text { Jas- } \\
\text { Jun }\end{array}$ & $\begin{array}{l}\text { Cam- } \\
\text { Bru }\end{array}$ & $\begin{array}{l}\text { Thy- } \\
\text { Hal }\end{array}$ & $\begin{array}{l}\text { Ros- } \\
\text { Hal }\end{array}$ & $\begin{array}{l}\text { Pis- } \\
\text { Hal }\end{array}$ & $\begin{array}{l}\text { Sar- } \\
\text { Hal }\end{array}$ & $\begin{array}{l}\text { Cor- } \\
\text { Psr }\end{array}$ & $\begin{array}{l}\text { Atl- } \\
\text { Psr }\end{array}$ & $\begin{array}{l}\text { Lav- } \\
\text { Psr }\end{array}$ & $\begin{array}{l}\text { Gen- } \\
\text { Psr }\end{array}$ & $\begin{array}{l}\text { Cen- } \\
\text { Pna }\end{array}$ & $\begin{array}{l}\text { Med- } \\
\text { Pna }\end{array}$ & $\begin{array}{l}\text { Pin- } \\
\text { Pna }\end{array}$ \\
\hline Eryngium campestre & . & 1 & 17 & . & 21 & . & 1 & 2 & . & . & 6 & 20 & 27 & 2 & . \\
\hline Dactylis glomerata & 40 & 42 & 25 & 50 & 18 & 21 & 15 & 18 & 23 & 2 & 38 & 25 & 73 & 23 & 55 \\
\hline
\end{tabular}

Mediterranean Pinus pinea forests

Phillyrea angustifolia

Pinion pineae

$\begin{array}{lll}\text { Eremopoa capillaris } & \cdot & 1 \\ \text { Trifolium tomentosum } & 1 & 1 \\ \text { Petrorhagia dubia } & 1 & 1 \\ \text { Filago arvensis } & \cdot & 1 \\ \text { Trifolium glomeratum } & \cdot & \cdot \\ \text { Aegilops triuncialis } & \cdot & 3 \\ \text { Anisantha sterilis } & 6 & 5 \\ \text { Trifolium arvense } & 1 & 7 \\ \text { Trifolium campestre } & 20 & 23 \\ \text { Campanula lyrata } & 1 & 7 \\ \text { Poa bulbosa } & 5 & 16 \\ \text { Silene italica } & 1 & 7 \\ \text { Micromeria myrtifolia } & 4 & 11\end{array}$

Diagnostic species for more than one alliance/community type

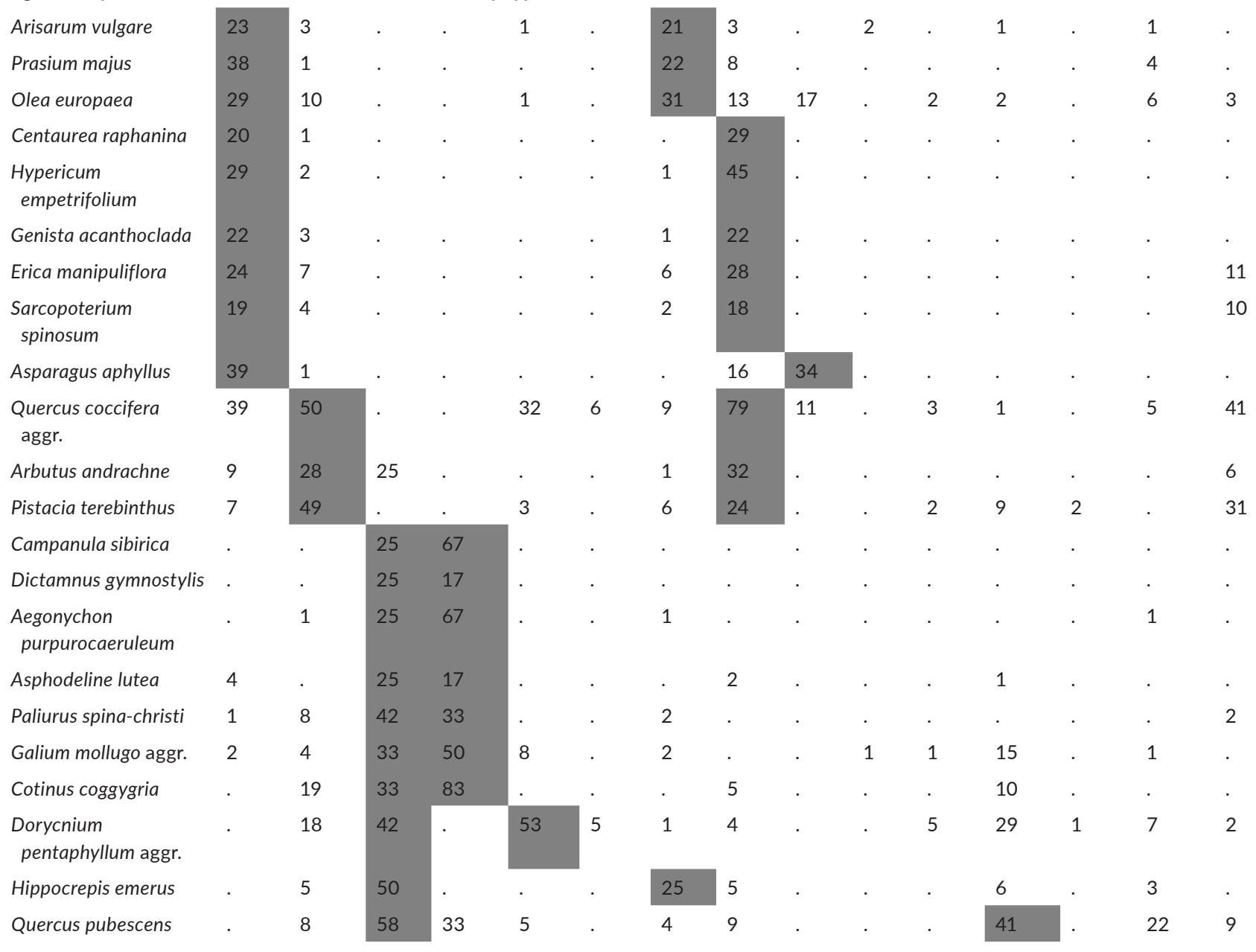


TABLE 2 (Continued)

\begin{tabular}{|c|c|c|c|c|c|c|c|c|c|c|c|c|c|c|c|}
\hline Alliance & $\begin{array}{l}\text { Pin- } \\
\text { Bru }\end{array}$ & $\begin{array}{l}\text { Sty- } \\
\text { Bru }\end{array}$ & $\begin{array}{l}\text { Jas- } \\
\text { Jun }\end{array}$ & $\begin{array}{l}\text { Cam- } \\
\text { Bru }\end{array}$ & $\begin{array}{l}\text { Thy- } \\
\text { Hal }\end{array}$ & $\begin{array}{l}\text { Ros- } \\
\text { Hal }\end{array}$ & $\begin{array}{l}\text { Pis- } \\
\text { Hal }\end{array}$ & $\begin{array}{l}\text { Sar- } \\
\text { Hal }\end{array}$ & $\begin{array}{l}\text { Cor- } \\
\text { Psr }\end{array}$ & $\begin{array}{l}\text { Atl- } \\
\text { Psr }\end{array}$ & $\begin{array}{l}\text { Lav- } \\
\text { Psr }\end{array}$ & $\begin{array}{l}\text { Gen- } \\
\text { Psr }\end{array}$ & $\begin{array}{l}\text { Cen- } \\
\text { Pna }\end{array}$ & $\begin{array}{l}\text { Med- } \\
\text { Pna }\end{array}$ & $\begin{array}{l}\text { Pin- } \\
\text { Pna }\end{array}$ \\
\hline $\begin{array}{l}\text { Rhaponticum } \\
\text { coniferum }\end{array}$ & . & . & . & . & 30 & 24 & . & . & . & . & 3 & 8 & 10 & 1 & . \\
\hline Brachypodium retusum & 36 & 1 & . & . & 51 & 1 & 39 & 63 & . & . & 4 & 19 & . & 7 & . \\
\hline Rosmarinus officinalis & . & 1 & . & . & 69 & 12 & 32 & . & 46 & . & 11 & 9 & 31 & 15 & . \\
\hline Genista hispanica & . & . & . & . & 17 & . & . & . & . & . & 1 & 20 & . & . & . \\
\hline Coronilla minima & . & . & . & . & 30 & 1 & . & . & . & . & 1 & 22 & 1 & 1 & . \\
\hline Fumana ericifolia & . & . & . & . & 19 & . & . & . & . & . & 1 & 14 & . & 1 & . \\
\hline Helianthemum italicum & . & . & . & . & 16 & . & 1 & . & . & . & . & 18 & . & 1 & . \\
\hline $\begin{array}{l}\text { Astragalus } \\
\text { monspessulanus }\end{array}$ & . & . & . & . & 14 & . & 1 & 2 & . & . & . & 17 & . & . & . \\
\hline $\begin{array}{l}\text { Aphyllanthes } \\
\text { monspeliensis }\end{array}$ & . & . & . & . & 43 & . & 1 & . & . & . & 3 & 28 & 1 & 1 & . \\
\hline $\begin{array}{l}\text { Brachypodium } \\
\text { phoenicoides }\end{array}$ & . & . & . & . & 27 & . & 2 & . & 3 & . & 1 & 32 & 1 & 5 & . \\
\hline Thymus vulgaris & . & . & . & . & 59 & . & 3 & . & . & . & 4 & 26 & 4 & 6 & . \\
\hline Odontites luteus & . & . & . & . & 15 & . & 1 & . & . & . & . & 13 & . & 4 & . \\
\hline Pistacia lentiscus & 48 & 5 & . & . & 14 & . & 89 & 36 & 57 & . & 1 & 10 & . & 41 & . \\
\hline Erica arborea & . & 2 & . & . & 3 & . & 39 & 3 & 3 & 3 & 13 & 56 & . & 24 & . \\
\hline Rubia peregrina & 1 & 2 & . & . & 21 & 1 & 56 & 15 & 39 & 19 & 29 & 71 & . & 51 & . \\
\hline Clematis flammula & . & 2 & . & . & 2 & 1 & 20 & 1 & . & . & 1 & 9 & . & 20 & 1 \\
\hline Rhamnus alaternus & 3 & 1 & . & . & 6 & . & 25 & 3 & 9 & . & 1 & 12 & . & 26 & . \\
\hline Daphne gnidium & . & 1 & . & . & 10 & . & 9 & 2 & 49 & 7 & 38 & 13 & . & 14 & . \\
\hline $\begin{array}{l}\text { Neoschischkinia } \\
\text { truncatula }\end{array}$ & . & . & . & . & . & . & . & . & . & 18 & 20 & . & . & . & . \\
\hline Cistus lasianthus & . & . & . & . & 1 & . & . & . & . & 27 & 20 & . & . & . & . \\
\hline Arenaria montana & . & . & . & . & 1 & . & . & . & . & 18 & 19 & . & . & . & . \\
\hline Teucrium scorodonia & . & . & . & . & 1 & . & . & . & . & 23 & 8 & 17 & . & 1 & . \\
\hline Calluna vulgaris & . & . & . & . & 1 & . & 1 & . & 15 & 57 & 9 & 28 & . & 4 & . \\
\hline Andryala integrifolia & . & . & . & . & . & . & . & . & 1 & 2 & 36 & 3 & 42 & 5 & 9 \\
\hline Jasione montana & . & . & . & . & 1 & . & 1 & . & 1 & 9 & 44 & 3 & 25 & 1 & . \\
\hline Rumex acetosella & . & 1 & . & . & . & . & . & . & 1 & 3 & 21 & 1 & 25 & 3 & 9 \\
\hline Lavandula pedunculata & . & . & . & . & 1 & . & . & . & 13 & . & 37 & . & 44 & . & 15 \\
\hline Cistus ladanifer & . & . & . & . & 2 & . & . & . & 16 & . & 32 & . & 40 & . & . \\
\hline Thymus mastichina & . & . & . & . & 3 & . & 1 & . & 16 & . & 27 & . & 28 & . & . \\
\hline Trifolium cherleri & 1 & 2 & . & . & . & . & . & . & . & . & 1 & . & 30 & . & 34 \\
\hline Trifolium stellatum & 9 & 5 & . & . & 1 & . & 3 & 3 & . & . & 1 & . & 30 & 1 & 32 \\
\hline Cynosurus echinatus & 1 & 11 & . & . & . & . & 4 & 8 & 1 & . & 23 & 1 & 33 & 6 & 63 \\
\hline Ornithopus compressus & . & 1 & . & . & . & . & . & 1 & 1 & . & 22 & . & 59 & 2 & 28 \\
\hline Phillyrea latifolia & 13 & 42 & . & . & . & 3 & 46 & 59 & . & . & . & 6 & . & 13 & 16 \\
\hline Cistus creticus & 43 & 54 & 17 & . & . & 16 & 22 & 68 & . & . & . & 3 & . & 15 & 93 \\
\hline Pteridium aquilinum & 1 & 4 & . & . & 1 & . & 1 & 2 & 4 & 68 & 40 & 36 & . & 4 & 14 \\
\hline Quercus ilex & 1 & 2 & . & . & 15 & 42 & 46 & 13 & . & 3 & 34 & 57 & 81 & 58 & . \\
\hline
\end{tabular}

Species occurring in $>300$ plots across the whole table except those already listed above

\begin{tabular}{|c|c|c|c|c|c|c|c|c|c|c|c|c|c|}
\hline Juniperus oxycedrus & 20 & 318 & 6 & 1 & 136 & 41 & 99 & 17 & 25 & 108 & 225 & 29 & 65 \\
\hline Teucrium polium aggr. & 19 & 205 & 9 & 1 & 53 & 4 & 32 & 32 & & 12 & 51 & 4 & 22 \\
\hline Briza maxima & 29 & 34 & . & . & 2 & . & 53 & 2 & 39 & 161 & 26 & 43 & 41 \\
\hline
\end{tabular}


TABLE 2 (Continued)

\begin{tabular}{|c|c|c|c|c|c|c|c|c|c|c|c|c|c|c|c|}
\hline Alliance & $\begin{array}{l}\text { Pin- } \\
\text { Bru }\end{array}$ & $\begin{array}{l}\text { Sty- } \\
\text { Bru }\end{array}$ & $\begin{array}{l}\text { Jas- } \\
\text { Jun }\end{array}$ & $\begin{array}{l}\text { Cam- } \\
\text { Bru }\end{array}$ & $\begin{array}{l}\text { Thy- } \\
\text { Hal }\end{array}$ & $\begin{array}{l}\text { Ros- } \\
\text { Hal }\end{array}$ & $\begin{array}{l}\text { Pis- } \\
\text { Hal }\end{array}$ & $\begin{array}{l}\text { Sar- } \\
\text { Hal }\end{array}$ & $\begin{array}{l}\text { Cor- } \\
\text { Psr }\end{array}$ & $\begin{array}{l}\text { Atl- } \\
\text { Psr }\end{array}$ & $\begin{array}{l}\text { Lav- } \\
\text { Psr }\end{array}$ & $\begin{array}{l}\text { Gen- } \\
\text { Psr }\end{array}$ & $\begin{array}{l}\text { Cen- } \\
\text { Pna }\end{array}$ & $\begin{array}{l}\text { Med- } \\
\text { Pna }\end{array}$ & $\begin{array}{l}\text { Pin- } \\
\text { Pna }\end{array}$ \\
\hline Crataegus monogyna & 5 & 115 & . & . & 9 & 1 & 15 & 5 & . & 20 & 62 & 142 & 4 & 39 & 11 \\
\hline $\begin{array}{l}\text { Geranium robertianum } \\
\text { aggr. (incl. Geranium } \\
\text { purpureum) }\end{array}$ & 56 & 59 & . & . & . & . & 49 & 13 & 9 & . & 30 & 35 & 19 & 29 & 7 \\
\hline
\end{tabular}

Aegean (Salvio fruticosae-Pinion brutiae), Anatolia and the Levant (Gonocytiso pterocladi-Pinion brutiae) and ultramafic substrates in southern Anatolia and Syria (Ptosimopappo-Quercion microphyllae). As these differences are not supported in our analysis, we include them in our geographically more widely conceived Pinion brutiae.

This alliance includes eastern Mediterranean Pinus brutia forests of the thermo- and mesomediterranean belts of Greece (mainland and Aegean islands), western and southern Anatolia, Cyprus, Lebanon and Syria, thriving on various substrates. Old-established reforestations within the supposed native distribution range of the dominant species can also occur, especially in mainland Greece and Anatolia. Besides Pinus brutia, also Cupressus sempervirens, Olea europaea and Quercus coccifera aggr. can be found in the tree layer. The shrub layer includes Juniperus phoenicea and Rhamnus lycioides. The herb and dwarf-shrub species with eastern Mediterranean distributions are numerous.

\subsection{2 | Meso- to supramediterranean Pinus brutia forests}

\section{Styraco officinalis-Pinion brutiae Bonari, M. Chytrý, Çoban, Kavgacı et Sağlam all. nov.}

Acronym: Sty-Bru; Figures 4; 5b; 6; 7; Cluster 4 p.p.

Nomenclatural type (holotypus): Verbasco pseudoholotrichi-Pinetum brutiae Vural, Akman et Quézel 1999 (Vural et al., 1999, p. 8)

Diagnostic species of the alliance: Alyssum strigosum, Brizochloa humilis, Crucianella latifolia, Daphne sericea, Eryngium falcatum, Fontanesia phillyreoides, Lathyrus aphaca, Lens ervoides, Phlomis samia, Quercus alnifolia, Quercus cerris, Quercus infectoria, Salvia tomentosa, Styrax officinalis, Thymbra spicata, Vicia tenuifolia + dalmatica.

This alliance includes the meso- and supramediterranean Pinus brutia forests of Anatolia, Levant, Cyprus, Crete and marginally also Greek mainland. Oak species such as Quercus alnifolia (in Cyprus), Quercus cerris and Quercus infectoria can be present in the tree layer. Arbutus andrachne, Daphne sericea, Fontanesia phillyreoides, Phillyrea latifolia, Quercus coccifera, Pistacia terebinthus, Styrax officinalis and Juniperus oxycedrus occur in the shrub layer. Mediterranean and eastern Mediterranean elements such as Alyssum strigosum and Eryngium falcatum characterize this alliance in the herb layer, which has a variable understorey due to the occurrence over a large area. It occurs on various substrates such as limestones, conglomerates, schists, marls and serpentinites. In the Taurus mountains, it is generally found up to $1,300-1,400 \mathrm{~m}$ a.s.l., extending inland through deep valleys in western and northern Anatolia reaching up to $800-1,000 \mathrm{~m}$ a.s.l. The forests of this alliance differ from the vicariant alliance Pinion brutiae, which is confined to lower elevations. Styraco-Pinion brutiae occurs in more favourable climatic conditions such as shorter summer drought, lower temperature and higher precipitation within meso- to supramediterranean elevational belts (Mayer \& Aksoy, 1998; Boydak et al., 2006).

\subsection{3 | Crimean Pinus brutia forests}

Jasmino fruticantis-Juniperion excelsae Didukh, Vakarenko et Shelyag-Sosonko ex Bonari et al. all. nov.

Acronym: Jas-Jun; Figures 4; 5c; 6; 7; Cluster 4 p.p.

Original diagnosis and diagnostic species: Didukh (1996, pp. 66-74) Nomenclatural type (holotypus): Phleo phleoidis-Juniperetum excelsae Didukh, Vakarenko et Shelyag-Sosonko in Didukh 1996 (Didukh, 1996, p. 73)

Synonyms: Jasmino-Juniperion excelsae Didukh, Vakarenko et Shelyag-Sosonko 1986 nom. inval. [ICPN Art. 2b]; Jasmino-Juniperion excelsae Didukh, Vakarenko et Shelyag-Sosonko ex Didukh 1996 nom. inval. [ICPN Art. 5]

Nomenclature comments: The proposal of the name JasminoJuniperion excelsae in Didukh et al. (1986) is invalid because the diagnosis does not contain any valid association name (i.e. types were not designated for the associations nor for the alliance). Didukh (1996) validated several associations within this alliance, most of them corresponding to Juniperus excelsa forests, but did not designate the type of the alliance, which remained invalid. The alliance was originally included in Fraxino orni-Cotinetalia (Quercetea pubescentis) but Mucina et al. (2016) moved it to Berberido creticae-Juniperetalia excelsae (Junipero-Pinetea sylvestris). The only association of the alliance including pine forests is the Achnathero bromoidis-Pinetum pityusae Didukh 1996 (Pinetum pityusae tauricum Didukh, Vakarenko et Shelyag-Sosonko 1986 nom. inval.). Although Achnatherum bromoides is not in the holotype of this association, the name is valid because this species is present in all the relevés ascribed to the association in Didukh et al. (1986), as part of the original diagnosis by unambiguous reference (hence ICPN Art. $3 f$ and Art. 16 do not apply). The name must be corrected [ICPN Art. 44] if we consider, following e.g. Euro+Med (2016-2020) and many other authors, that Pinus pityusa 
is a later synonym of Pinus brutia: Achnathero bromoidis-Pinetum brutiae Didukh 1996 nom. corr. (=Achnathero bromoidis-Pinetum pityusae Didukh 1996 nom. inept.). Only the Pinus brutia forests belonging to this association have been included and analysed in this study. Diagnostic species of the alliance: Achnatherum bromoides, Allium carinatum, Asparagus verticillatus, Asperula tenella, Astragalus hamosus, Bothriochloa ischaemum, Bromopsis cappadocica, Bupleurum falcatum, Carex caryophyllea, Carex flacca, Carex halleriana, Centaurea diffusa, Centaurea sterilis, Cleistogenes serotina, Convolvulus cantabrica, Convolvulus lineatus, Diplotaxis tenuifolia, Elymus nodosus, Erysimum cuspidatum, Festuca stricta, Fibigia clypeata, Fumana arabica, Fumana procumbens, Galatella villosa, Galium biebersteinii, Gaudiniopsis macra, Helianthemum stevenii, Hieracium $\times$ brachiatum, Inula aspera, Inula ensifolia, Inula oculus-christi, Iris pumila, Jasminum fruticans, Juniperus excelsa, Jurinea ledebourii, Linum austriacum, Linum nodiflorum, Linum strictum aggr. (incl. Linum corymbulosum), Melica ciliata, Melica transsilvanica, Odontarrhena tortuosa, Orchis simia, Ornithogalum pyrenaicum, Piptatherum holciforme, Pistacia atlantica, Pistorinia hispanica, Poa sterilis, Podospermum laciniatum, Polygala major, Psephellus declinatus, Reseda lutea, Rhus coriaria, Ruscus aculeatus, Salvia officinalis, Scorzonera crispa, Seseli dichotomum, Sorbus aucuparia, Stipa lessingiana, Stipa pennata aggr. (incl. Stipa eriocaulis), Teucrium chamaedrys, Teucrium polium aggr., Thymus roegneri, Veronica multifida, Viola odorata.

The Crimean Pinus brutia forests occur in few localities along a narrow coastal belt on the southern slopes of the Crimean Mountains. They are characterized by a mixture of Mediterranean and non-Mediterranean elements. Juniperus excelsa, Pistacia atlantica and Quercus pubescens are found in the tree layer. Jasminum fruticans and Paliurus spina-christi frequently occur in the shrub layer. The herb layer is rich in both annual and perennial species, containing Mediterranean elements such as Achnatherum bromoides and Carex halleriana. These forests show floristic relations with the other two alliances that occur in the Black Sea area (Styraco officinalis-Pinion brutiae and Campanulo sibiricae-Pinion brutiae) although showing differential elements. Further analyses including the other local forest types are needed to clarify the contentious hierarchical position of the alliance Jasmino fruticantis-Juniperion excelsae.

\subsubsection{Caucasian Pinus brutia forests}

Campanulo sibiricae-Pinion brutiae Litvinskaya et Postarnak ex Mucina in Mucina et al. 2016

Acronym: Cam-Bru; Figures 4; 5d; 6; 7; Cluster 4 p.p.

Nomenclatural type (holotypus): Epimedio colchici-Pinetum pythiusae Litvinskaya et Postarnak ex Mucina in Mucina et al. 2016

Synonym: Campanulo longistylae-Pinion pithyusae Litvinskaya et Postarnak 2002 nom. inval. [ICPN Art. 5]

This alliance groups the Pinus brutia forests occurring on calcareous substrates in a narrow belt along the northwest Caucasian Black Sea coast. The alliance is characterized by non-Mediterranean elements. Carpinus orientalis and Quercus pubescens are found in the tree layer. Cotinus coggygria and Epimedium pinnatum frequently occur in the shrub layer. The herb layer is rich in Colchic elements.

\subsection{5 | Mesomediterranean Pinus halepensis forests}

Thymo vulgaris-Pinion halepensis Biondi et Pesaresi in Pesaresi et al. 2017

Acronym: Thy-Hal; Figures 4; 5e; 6; 7; Clusters $9+10$

Nomenclatural type (holotypus): Cisto albidi-Pinetum halepensis Vagge, Biondi et Pesaresi in Pesaresi et al. 2017

This alliance comprises Pinus halepensis forests widely distributed in eastern Spain and extending to southeastern France (Languedoc-Roussillon and Provence), northeastern Italy (Liguria) and the Balearic Islands, mainly on base-rich substrates. Its distribution matches well with the Mediterranean basophilous scrub, rich in perennial herbs, of the order Rosmarinetalia, and in part with the sclerophyllous forests of the alliance Quercion ilicis. The climatic conditions in this area allow the development under the pine canopy of the scrub and some perennial herbs of the alliance Brachypodion phoenicoidis. The tree layer is dominated by Pinus halepensis. Juniperus phoenicea and Juniperus oxycedrus can occur in the shrub layer. Species of the western mesomediterranean element are more common than in the Pistacio lentisci-Pinion halepensis, with which this alliance partially overlaps. Aphyllanthes monspeliensis, Brachypodium phoenicoides, Cistus albidus, Genista scorpius, Helichrysum stoechas, Lavandula latifolia and Thymus vulgaris are frequent in the low shrub and herb layers.

\subsection{6 | Meso- to supramediterranean forests and pre-forests of North Africa}

Rosmarino eriocalycis-Pinion halepensis Bonari, M. Chytrý et Fernández-González all. nov.

Acronym: Ros-Hal; Figures 4; 5f; 6; 7; Clusters $7+8$

Nomenclatural type (holotypus): Genisto quadriflorae-Pinetum halepensis Meddour, Meddour-Sahar, Zeraia et Mucina in Bonari et al. 2021

Diagnostic species of the alliance: Alyssum granatense, Ammoides atlantica, Ampelodesmos mauritanicus, Anisantha rubens, Arabis nova, Bombycilaena discolor, Bufonia tenuifolia, Bupleurum atlanticum, Catapodium marinum, Centaurea boissieri, Cistus clusii, Dianthus caryophyllus, Ebenus pinnata, Echinaria capitata, Eruca vesicaria, Filago pyramidata, Fumana fontanesii, Genista capitellata, Globularia alypum, Helianthemum cinereum, Helianthemum virgatum, Herniaria hirsuta, Hornungia petraea, Macrochloa tenacissima, Minuartia montana, Odontarrhena alpestris, Papaver hybridum, Paronychia argentea, Petrorhagia illyrica, Pilosella pseudopilosella, Rosmarinus eriocalyx, Schismus barbatus, Tetraclinis articulata, Teucrium pseudochamaepitys, Thymelaea virescens, Thymus algeriensis, Thymus munbyanus.

Type relevé (holotypus) of Genisto quadriflorae-Pinetum halepensis Meddour, Meddour-Sahar, Zeraia et Mucina ass. nov.: Benabdeli (1996, p. 110, Table 4, relevé 3) 
This alliance includes forests and pre-forests dominated by Pinus halepensis in Algeria, Morocco and partly Tunisia. It occurs in semiarid and subhumid climates. Most of the stands occur in the mesomediterranean belt, but some are reaching the supramediterranean belt, in particular in the eastern part of the Atlas. The tree layer is dominated by Pinus halepensis. Junipers (Juniperus oxycedrus and Juniperus phoenicea) can occur in the shrub layer. The understorey is characterized by Cistus clusii, Globularia alypum, Helianthemum cinereum, Macrochloa tenacissima, Rosmarinus eriocalyx and Thymus munbyanus.

The European vegetation checklist (Mucina et al., 2016) does not include Algeria, Morocco and Tunisia, where these pine (pre-)forests occur. As highlighted first by Fennane (2003) and then by Meddour et al. (2017), the syntaxonomical status of Quercetea ilicis and lowgrown matorral, macchia and garrigue (Pistacio lentisci-Rhamnetalia alaterni) is unclear for the whole of North Africa, and a critical revision is needed. This alliance contributes to closing this gap. These (pre-)forest formations with tall Pinus halepensis individuals occur over a large area of North Africa. This alliance, with the presence of North African species, is the African vicariant of the Pistacio lentisci-Pinion halepensis and Thymo vulgaris-Pinion halepensis.

\subsection{7 | Thermomediterranean Pinus halepensis forests}

Pistacio lentisci-Pinion halepensis Biondi, Blasi, Galdenzi, Pesaresi et Vagge in Biondi et al. 2014

Acronym: Pis-Hal; Figures 4; 5g; 6; 7; Clusters 5 p.p. +6

Nomenclatural type (holotypus): Pistacio lentisci-Pinetum halepensis De Marco, Veri et Caneva 1984

Synonym: Rosmarino officinalis-Pinion halepensis Biondi et Pesaresi in Pesaresi et al. 2017

Nomenclature comments: De Marco et al. (1984) did not designate a holotype for the association, but as they designated holotypes for the three subassociations and they said (p. 29) that the subassociation pinetosum "represents the typical aspect of the association," the holotype of the subassociation pinetosum automatically becomes the holotype of the association [ICPN Art. 5b, §3]. We consider the alliance Rosmarino officinalis-Pinion halepensis as a synonym of Pistacio lentisci-Pinion halepensis, because we did not support it by numerical analysis of a much larger data set than the one used in the study containing the original description of this alliance (Pesaresi et al., 2017).

This broadly distributed Mediterranean alliance includes Pinus halepensis forests of the thermomediterranean belt, from mainland Greece to eastern Spain and probably also some Mediterranean coastal areas of northwestern Africa. These forests thrive on various substrates, mostly calcareous, and can be locally co-dominated by other thermophilous pine species. This vegetation type also includes pine plantations. Quercus ilex may occur beside Pinus halepensis. Common Mediterranean shrubs and dwarf shrubs such as Myrtus communis, Pistacia lentiscus, Rhamnus alaternus and Rosmarinus officinalis are present, often with lianas such as Rubia peregrina and Smilax aspera. The herb layer is generally species-poor, but Mediterranean elements such as Brachypodium retusum are common.

\subsection{8 | Thermo- to mesomediterranean Pinus halepensis forests of Greece}

\section{Sarcopoterio spinosi-Pinion halepensis Biondi et Pesaresi in Pesaresi} et al. 2017

Acronym: Sar-Hal; Figures 4; 5h; 6; 7; Cluster 5 p.p.

Nomenclatural type (holotypus): Anthyllido hermanniae-Pinetum halepensis Biondi et Pesaresi in Pesaresi et al., 2017

Nomenclature comments: There is a prior valid alliance name (Alkanno baeoticae-Pinion halepensis Mucina et Dimopoulos in Mucina et al. 2009) described for the Aegean Pinus halepensis forests on ultramafic substrates. However, our database did not give support to the differentiation of edaphic alliances, possibly due to the underrepresentation of plots from ultramafic substrates. Therefore, we prefer to adopt the alliance Sarcopoterio spinosi-Pinion halepensis for the Aegean Pinus halepensis forests included here, assuming that the independence of the Alkanno baeoticae-Pinion halepensis can be corroborated with new data.

This alliance includes thermo- to mesomediterranean Pinus halepensis forests in mainland Greece and in some Aegean islands (Konstantinidis et al., 2012). Besides Pinus halepensis, Quercus coccifera can occur in the low tree layer. In the shrub layer, Mediterranean elements such as Arbutus andrachne, Arbutus unedo, Pistacia lentiscus and Pistacia terebinthus occur. Anthyllis hermanniae, Centaurea raphanina, Cistus creticus, Crepis fraasii, Cyclamen graecum, Erica manipuliflora, Genista acanthoclada and Hypericum empetrifolium are present in the herb layer. These pine forests grow mainly on calcareous substrates.

\subsubsection{Atlantic Pinus pinaster and Pinus pinea forests on sand}

Coremato albi-Pinion pinastri J.C. Costa, Neto, Capelo, Aguiar, Monteiro-Henriques et Bonari all. nov.

Acronym: Cor-Psr; Figures 4; 5i; 6; 7; Cluster 12

Nomenclatural type (holotypus): Pinetum pinastro-pineae J.C. Costa et Neto in Bonari et al. 2021

Diagnostic species of the alliance: Acacia longifolia, Andryala arenaria, Aristolochia baetica, Armeria macrophylla, Armeria velutina, Asparagus albus, Carlina hispanica, Carpobrotus edulis, Centaurea sphaerocephala, Chamaerops humilis, Cistus calycinus, Cistus crispus, Cistus halimifolius, Cistus libanotis, Cistus salviifolius, Corema album, Cytisus grandiflorus, Euphorbia baetica, Euphorbia portlandica, Galium minutulum, Genista triacanthos, Helichrysum italicum, Iris xiphium, Juniperus phoenicea, Lagurus ovatus, Lavandula stoechas, Morella faya, Osyris lanceolata, Retama monosperma, Scrophularia canina, Seseli tortuosum, Thymus albicans, Thymus capitellatus, Ulex argenteus, Ulex boivinii, Ulex genistoides, Ulex parviflorus. 
Type relevé (holotypus) of Pinetum pinastro-pineae J.C. Costa et Neto ass. nov.: Pinus pinea 4, Pinus pinaster 2, Pistacia lentiscus 2, Ulex parviflorus subsp. parviflorus 2, Daphne gnidium 2, Corema album 2, Juniperus phoenicea subsp. turbinata 1, Cistus halimifolius 1, Ulex genistoides +, Cistus calycinus + , Seseli tortuosum + , Helichrysum italicum subsp. picardii + , Cistus salviifolius 1 , Asparagus aphyllus 1, Thymus capitellatus 1, Lavandula pedunculata subsp. lusitanica 1, Carpobrotus edulis +, Carlina hispanica +, Corynephorus canescens +, Dactylis glomerata subsp. hispanica +, Sedum sediforme + , Centaurea sphaerocephala + , Calluna vulgaris + . Area: $60 \mathrm{~m}^{2}$; elevation: $10 \mathrm{~m}$ a.s.l.; aspect: W; slope: $6^{\circ}$; locality: Praia das Bicas, Meco (Sesimbra, Portugal); coordinates: WGS84 $38.49041^{\circ} \mathrm{N}, 9.18135^{\circ} \mathrm{W}$.

This alliance includes Atlantic southwestern Iberian natural forests dominated by Pinus pinaster and/or Pinus pinea occurring mostly on sandy soils close to the coast, although our data show a higher constancy of Pinus pinea over Pinus pinaster. This is related to a disproportion of vegetation plots in the data set that deviate from reality. Pistacia lentiscus and Juniperus phoenicea (subsp. turbinata) can occur in the shrub layer. Cistus calycinus, Corema album, Cytisus grandiflorus, Helichrysum italicum, Morella faya, Osyris lanceolata, Seseli tortuosum and Ulex genistoides are taxa relatively restricted to such coastal areas and present in the understorey. By studying in situ macroremains, García-Amorena et al. (2007) showed that communities dominated by Pinus pinaster thrived in these sandy coastal sites at least during the first half of the Holocene (7,930-7,430 cal years BP). Co-occurring with Pinus pinaster, Pinus pinea macroremains and charcoals are more frequent in the region to the south of Lisbon and have been dated as far back as 6,300-6,400 ${ }^{14} \mathrm{C}$ years BP (Carrión Marco, 2005). Old-established plantations in these coastal environments are indistinguishable from naturally established communities based on their floristic composition. We hypothesize that for long these communities have been shaped by the effect of the cold water of the Atlantic Ocean, which influences local temperature and summer fogginess. Furthermore, strong sea currents and powerful storms support sand deposition, which extends far inland. However, inland plantations, even if old-established, lack the above-mentioned taxa and cannot be considered a part of this alliance. Although Pinus pinaster (and possibly Pinus pinea) was common in inland communities in pre-Holocene times, it declined during the Holocene, being progressively replaced by other Mediterranean species (Figueiral, 1995). Acacia longifolia and Carpobrotus edulis are alien species invading these native communities and displacing native plant taxa. The new association describes communities dominated by Pinus pinaster and Pinus pinea, enduring strong maritime influence, on deep sandy soils of southwestern Portugal, under an upper thermomediterranean, dry to subhumid bioclimate.

\subsubsection{0 | Atlantic Pinus pinaster forests}

This group includes Atlantic acidophilous Pinus pinaster forests of the northwestern Iberian Peninsula and along the Gulf of Biscay. They are largely old-established plantations. The tree layer is dominated by Pinus pinaster, but Quercus robur, Quercus suber or Frangula alnus can also occur. The shrub layer can contain Erica cinerea, Erica scoparia and Ulex europaeus, while the low shrub and herb layer comprises Calluna vulgaris, Daboecia cantabrica, Erica umbellata, Pteridium aquilinum and Ulex minor.

\subsubsection{1 | Meso- to supramediterranean Central Iberian Pinus pinaster forests}

Lavandulo pedunculatae-Pinion pinastri Fernández-González, Bonari et M. Chytrý all. nov.

Acronym: Lav-Psr; Figures 4; 5k; 6; 7; Cluster 16

Nomenclatural type (holotypus): Centaureo albae-Pinetum pinastri Fernández-González, Bonari et Chytrý in Bonari et al. 2021

Nomenclature comments: The alliance "Pino pinastri-Juniperion phoeniceae Pérez Latorre et Cabezudo in Pérez Latorre et al. 1998" ("Junipero phoeniceae-Pinion acutisquamae Pérez Latorre et Cabezudo in Pérez Latorre et al. 1998 corr. Rivas-Martínez et al. 2002 nom. inv. propos.") is accepted by Mucina et al. (2016) for "southern Iberian shrublands on dolomitic and ultramafic substrates" and by Rivas-Martínez et al. (2011) for "Betic juniper shrublands and pine forests" on the same substrates. Nevertheless, this alliance name is invalid because the holotype designated (Pino pinastri-Quercetum cocciferae Cabezudo et al., 1989) is an invalid association as the authors (Cabezudo et al., 1989) wrongly designated two different holotype relevés (ICPN Art. 5). Moreover, most of the relevés of this thermomediterranean association do not correspond to pine forests and their floristic composition differs considerably from the alliance we are proposing here.

Diagnostic species of the alliance: Agrostis castellana + tenuis, Anarrhinum bellidifolium, Aristolochia paucinervis, Arnoseris minima, Arrhenatherum elatius, Campanula lusitanica, Centaurea alba aggr., Centaurea langei, Cistus ocymoides, Cistus populifolius, Cistus psilosepalus, Cistus umbellatus, Clinopodium vulgare, Coronilla repanda, Cytisus multiflorus, Cytisus scoparius, Cytisus striatus, Digitalis thapsi, Erica australis, Festuca elegans, Filago minima, Genista falcata, Genista florida, Genista tridentata, Geum sylvaticum, Holcus lanatus, Holcus mollis, Hypericum linarifolium, Hypochaeris radicata, Lotus parviflorus, Luzula lactea, Micropyrum tenellum, Quercus faginea, Quercus pyrenaica, Teesdalia nudicaulis, Thapsia minor, Tuberaria lignosa.

Type relevé (holotypus) of Centaureo albae-Pinetum pinastri FernándezGonzález, Bonari et M. Chytrý ass. nov. (Braun-Blanquet scale): Pinus pinaster 4, Cistus ladanifer subsp. ladanifer 2, Lavandula pedunculata 1, Origanum vulgare subsp. virens 1, Rosmarinus officinalis 1, Daphne gnidium 1, Phillyrea angustifolia +, Pistacia terebinthus +, Cytisus scoparius +, Quercus faginea subsp. broteroi +, Quercus pyrenaica + , Retama sphaerocarpa + , Rubia peregrina + , Thymus mastichina subsp. mastichina + , Agrostis castellana + , Andryala integrifolia + , Bituminaria bituminosa + , Briza maxima + , Carex distachya + , Carlina hispanica + , Centaurea alba 

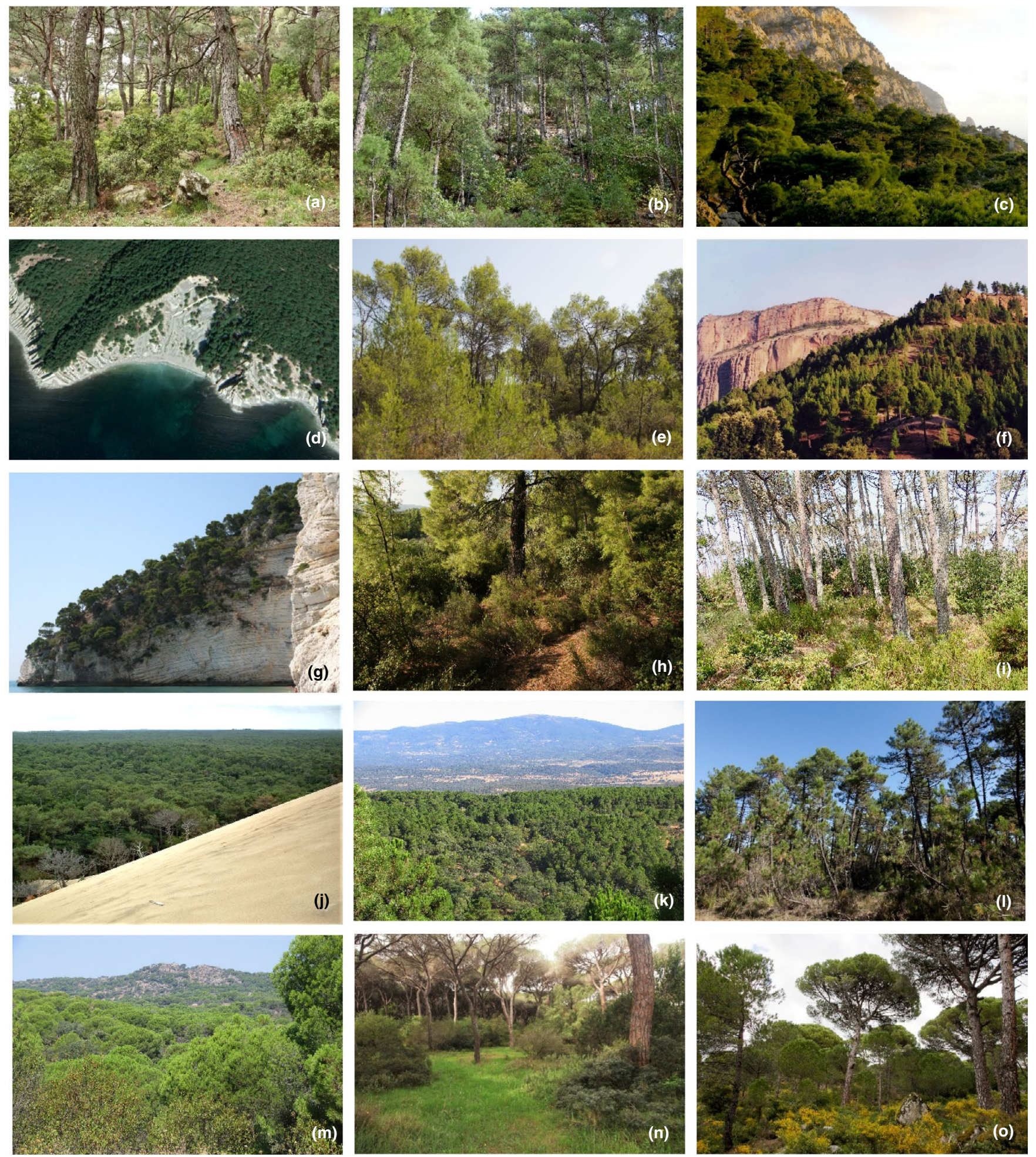

FIGURE 5 Typical stands of each alliance or informal group of communities. a = Pinion brutiae (Yamanlar Dağı, Izmir, Turkey); b = Styraco officinalis-Pinion brutiae (Cehennemdere, Mersin, Turkey); c = Jasmino fruticantis-Juniperion excelsae (Cape Aya Reserve, Sevastopol, Crimea); $\mathrm{d}=$ Campanulo sibiricae-Pinion brutiae (Golubaya Dolina, Krasnodar, Russian Federation); e = Thymo vulgaris-Pinion halepensis (Combe des Pins, Le Triadou, France); $\mathrm{f}=$ Rosmarino eriocalycis-Pinion halepensis (Tamga forest, High Atlas of M'goun, Morocco); $\mathrm{g}=$ Pistacio lentisci-Pinion halepensis (Mattinata, Foggia, Italy); $\mathrm{h}=$ Sarcopoterio spinosi-Pinion halepensis (Neos Marmaras, Sithonia Peninsula, Greece); $\mathrm{i}=$ Coremato albi-Pinion pinastri (Praia do Pedrógão, Leiria, Portugal); j = Atlantic Pinus pinaster forests (Dune of Pilat, Landes, France); $\mathrm{k}=$ Lavandulo pedunculatae-Pinion pinastri (Pedro Bernardo, Ávila, Spain); I = Genisto pilosae-Pinion pinastri (Monticiano, Siena, Italy); $m$ = Central lberian Pinus pinea forests (Almorox, Toledo, Spain); $\mathrm{n}=$ Mediterranean Pinus pinea forests (Castiglione della Pescaia, Grosseto, Italy); o = Pinion pineae (Kozak-Kaplan Köyü, Bergama, Turkey). Photo credits: O. Argagnon (e), M. Beskaravayny (c), G. Bonari (a, g, h, I, n), A. Çalışkan (b), M. Chytrý (o), J. El Oualidi (f), P. M. Fernandes (j), F. Fernández-González (k, m), Maxar Technologies, Google Earth 2020 ${ }^{\odot}$ (d), T. Monteiro-Henriques (i) 
subsp. alba +, Dactylis glomerata subsp. hispanica + , Holcus lanatus + , Hypericum linarifolium + , Plantago lanceolata + , Pteridium aquilinum + , Sanguisorba verrucosa +, Trifolium arvense + . Area: $150 \mathrm{~m}^{2}$; elevation: 740 m a.s.l.; aspect: S; slope: $10^{\circ}$; locality: Piedralaves (Ávila, Spain), coordinates: WGS84 $40.3146^{\circ} \mathrm{N} ; 4.7255^{\circ} \mathrm{W}$.

This group comprises meso- and supramediterranean Pinus pinaster forests in the central Iberian Peninsula, distributed mainly on the northern Castilian plateau and the adjacent mountain systems (Central System and Iberian System), extending south to some Andalusian mountains. The elevational range is $400-1,500 \mathrm{~m}$. They thrive mainly on siliceous substrates (granite, sandstone, quartzite and other metamorphic rocks, as well as sedimentary deposits related to them) or partially decalcified soils on limestones. The tree layer is dominated by Pinus pinaster, but Quercus rotundifolia and Quercus pyrenaica can occur and are the main competing trees. Cistus ladanifer, Cytisus scoparius, Daphne gnidium, Erica australis, Genista tridentata, Lavandula pedunculata and Thymus mastichina are frequent in the shrub layer. Hypochaeris radicata, Jasione montana or Pteridium aquilinum are present in the herb layer. Although Pinus pinaster is considered native in most of these areas, it has also been used often in forestry plantations in Spain for the last 80 years. The association Centaureo albae-Pinetum pinastri corresponds to the maritime pine forests of the southern, mesomediterranean slopes of the Sierra de Gredos (Central System), where a continuous pine cover is documented in the palaeopalynological record for at least the last 2,500 years (López-Sáez et al., 2010).

\subsubsection{2 | Thermo- to mesomediterranean Pinus pinaster forests}

\section{Genisto pilosae-Pinion pinastri Biondi et Vagge 2015}

Acronym: Gen-Psr; Figures 4; 5I; 6; 7; Cluster 13

Nomenclatural type (holotypus): Erico scopariae-Pinetum pinastri Biondi et Vagge 2015

This alliance includes thermo- to mesomediterranean forests of Pinus pinaster of the Ligurian, Provencal and Languedoc seaboards and Corsica. It is characterized by a mixture of Mediterranean and non-Mediterranean species, but an impoverishment in Mediterranean sclerophyllous shrubs is noteworthy. It occurs on a variety of soils, including those on serpentinite, but mainly acidic or decalcified. The tree layer can contain an admixture of broad-leaved trees such as Castanea sativa, Fraxinus ornus, Quercus ilex and Quercus pubescens. In the shrub layer, Arbutus unedo, Erica arborea, Erica scoparia, Juniperus communis and Juniperus oxycedrus can be found. Brachypodium phoenicoides, Calluna vulgaris, Cistus salviifolius, Pteridium aquilinum and Teucrium chamaedrys are frequent in the low shrub and herb layers.

\subsubsection{3 | Mesomediterranean Central Iberian Pinus pinea forests}

Acronym: Cen-Pna; Figures 4; 5m; 6; 7; Cluster 15
This group includes Pinus pinea forests of the central Iberian Peninsula (Castilian plateaus and low hills of the Central System), mostly in the mesomediterranean belt (elevations of 500-1,000 m). They grow mainly on fluvial or aeolian sand deposits and other coarse-textured soils on siliceous rocks. Pinus pinea is considered native here, as in the southwestern and western coast of the Iberian Peninsula (Loidi, 2017; Mutke et al., 2019), although most of these forests have been intensively managed for wood, pine seed harvesting and livestock grazing. The tree layer is dominated by Pinus pinea, sometimes with Pinus pinaster, but Quercus rotundifolia is common in the understorey. Juniperus oxycedrus and Retama sphaerocarpa can occur in the shrub layer. Dwarf shrubs such as Lavandula pedunculata, Rosmarinus officinalis, or rockroses (Cistus ladanifer) are present. In the open and grazed stands, the herb layer is rich in Mediterranean annuals, which dominate among the diagnostic species of the group as differentiated by TWINSPAN, but otherwise it is floristically and biogeographically related to the herb layers of the Central Iberian Pinus pinaster forests. Indeed, Pinus pinaster forests (see paragraph 3.1.11) are much more extended in this large area of central Spain, overlapping their distribution with Pinus pinea forests, and there are mixed forests with both pines at low elevations of the Central System and on the sedimentary deposits of the Castilian plateaus. Hence the distinction between Pinus pinea and Pinus pinaster forests in central lberia could be reflected at the level of associations rather than of alliances.

\subsubsection{4 | Thermomediterranean Pinus pinea forests}

Acronym: Med-Pna; Figures 4; 5n; 6; 7; Cluster 11 p.p.

This group includes thermomediterranean, partly supposedly native forests but largely old-established plantations of Pinus pinea in Catalonia, France, the Italian Peninsula, Sicily and Sardinia. Pinus pinea old-established plantations on the Italian Peninsula occur at the sea level on sand. In the hinterland, reforested areas with this pine species are few. Catalonian, Provencal and Languedoc forests can reach up to $800 \mathrm{~m}$. The populations of Pinus pinea on sandstone in the Provence are likely native (Quézel, 1979), as well as those at one site in Sardinia (Arrigoni, 1967). However, the areas currently occupied by Pinus pinea have been artificially extended (and often heavily managed) in recent times. The structure and floristic composition of these forests is highly influenced by management and human impact (Bonari et al., 2019a). In the tree layer, Quercus ilex and Quercus pubescens can occur. More frequent species in the shrub layer include Erica arborea, Phillyrea angustifolia, Pistacia lentiscus and Rhamnus alaternus, but also lianas (Rubia peregrina, Smilax aspera). The herb layer contains Asparagus acutifolius, Brachypodium sylvaticum and Cistus salviifolius.

\subsubsection{5 | Eastern thermo- to mesomediterranean Pinus pinea forests}

Pinion pineae Feinbrun 1959

Acronym: Pin-Pna; Figures 4; 5o; 6; 7; Cluster 3 
TAB LE 3 Shortened synoptic table of the percentage constancies of the diagnostic and most frequent species for the vegetation plots classified to the EUNIS habitat types corresponding to the classes Pinetea halepensis and Quercetea ilicis

\begin{tabular}{|c|c|c|c|}
\hline Species & No. of plots & Pinetea halepensis (T3A) & Quercetea ilicis (T21) \\
\hline \multicolumn{4}{|l|}{ Tree species } \\
\hline Pinus halepensis & 973 & 48 & 8 \\
\hline Pinus pinaster & 563 & 29 & 4 \\
\hline Quercus coccifera & 1,127 & 31 & 23 \\
\hline Quercus ilex & 2,158 & 32 & 59 \\
\hline Quercus rotundifolia & 806 & 7 & 25 \\
\hline Quercus suber & 525 & 6 & 15 \\
\hline Acer monspessulanum & 185 & 1 & 6 \\
\hline Cistus creticus & 491 & 18 & 7 \\
\hline Teucrium polium aggr. & 344 & 14 & 5 \\
\hline Dorycnium pentaphyllum & 486 & 18 & 7 \\
\hline Helichrysum stoechas & 298 & 12 & 4 \\
\hline \multicolumn{4}{|c|}{ Diagnostic species for Quercetea ilicis (T21) } \\
\hline Ruscus aculeatus & 1,277 & 10 & 40 \\
\hline Asplenium adiantum-nigrum & 817 & 4 & 27 \\
\hline Hedera helix aggr. & 1,136 & 11 & 34 \\
\hline Rubia peregrina & 2,555 & 41 & 68 \\
\hline Phillyrea latifolia & 1,407 & 23 & 38 \\
\hline Cyclamen repandum & 288 & 2 & 9 \\
\hline Helleborus foetidus & 150 & 1 & 5 \\
\hline Luzula forsteri & 250 & 2 & 8 \\
\hline \multicolumn{4}{|c|}{ Species occurring in $>1,000$ plots across the whole table except the species already listed above } \\
\hline Asparagus acutifolius & 1,652 & 32 & 41 \\
\hline Smilax aspera & 1,573 & 31 & 39 \\
\hline Arbutus unedo & 1,235 & 22 & 32 \\
\hline Brachypodium retusum & 1,231 & 32 & 26 \\
\hline Juniperus oxycedrus aggr. & 1,211 & 34 & 24 \\
\hline Pistacia lentiscus & 1,150 & 32 & 23 \\
\hline Erica arborea & 1,009 & 17 & 26 \\
\hline
\end{tabular}

Nomenclatural type (holotypus): Pinetum pineae libanoticum Feinbrun 1959

This alliance includes native forests of Pinus pinea in the eastern Mediterranean and the eastern Euxinian region, occurring in the thermo- to mesomediterranean belts. These forests are chiefly present on acidic rocky substrates. The shrub layer includes Arbutus unedo, Cistus creticus, Erica manipuliflora, Lavandula stoechas and Quercus coccifera. As in other Pinus pinea forests, the herb layer is rich in annual species. However, perennial species are also present (Dianthus strictus, Genista lydia, Micromeria myrtifolia). 


\subsection{Climatic and elevational patterns}

The individual alliances of Mediterranean pine forests mostly occupy distinct elevational ranges (Figure 6) and are related to different climatic features (Figures 7 and 8). The climatic conditions relate to thermo-, meso- and supramediterranean belts. Precipitation seasonality, temperature and temperature seasonality are related to the differentiation of the alliances along the first ordination (DCA) axis of species composition, while precipitation is related to the second axis.

The alliances of the Pinus brutia forests differ in their typical elevations, with Pinion brutiae being confined to the thermo- and mesomediterranean belts (Figure 6), with high temperature and low precipitation (Figure 7a and c), as opposed to the Styraco officinalis-Pinion brutiae, which tends to be more frequent at higher elevations (Figure 6). The Jasmino fruticantis-Juniperion excelsae is restricted to a small area, but its climatic relationships seem to be intermediate between those of the other two alliances, while Campanulo sibiricae-Pinion halepensis shows a relationship to higher precipitation (Figure 7a). The alliances dominated by Pinus halepensis also differ in their elevational range, although Sarcopoterio spinosi-Pinion halepensis occurs in similar elevational belts as Pistacio lentisci-Pinion halepensis and Thymo vulgaris-Pinion halepensis, but differs in terms of precipitation seasonality (Figures 6 and 7b). The Pistacio lentisci-Pinion halepensis is typical of the thermomediterranean belt with a warmer climate and more seasonal precipitation, while Thymo vulgaris-Pinion halepensis occurs mainly in the mesomediterranean belt (Figures 6 and 7b). Rosmarino eriocalycis-Pinion halepensis occurs mainly in the meso- to supramediterranean belts, with a high temperature seasonality (Figures 6 and 7d). Non-coastal alliances of Pinus pinaster forests (Genisto pilosae-Pinion pinastri and Lavandulo pedunculatae-Pinion pinastri) show a different elevational pattern between the thermo- and mesomediterranean belts, respectively. Their climatic patterns are similar, while Coremato albi-Pinion pinastri and the Atlantic Pinus pinaster forests show opposite trends of precipitation and precipitation seasonality at low elevations (Figures 6, 7a and b). Also, Pinus pinea alliances occur at different elevations, but while the Mediterranean Pinus pinea communities, including old-established plantations, are concentrated in coastal areas, native forests of Pinion pineae in the eastern Mediterranean are more frequently found at higher elevations of the meso- (to supra-)mediterranean belt with high precipitation seasonality (Figures 6 and $7 \mathrm{~b}$ ).
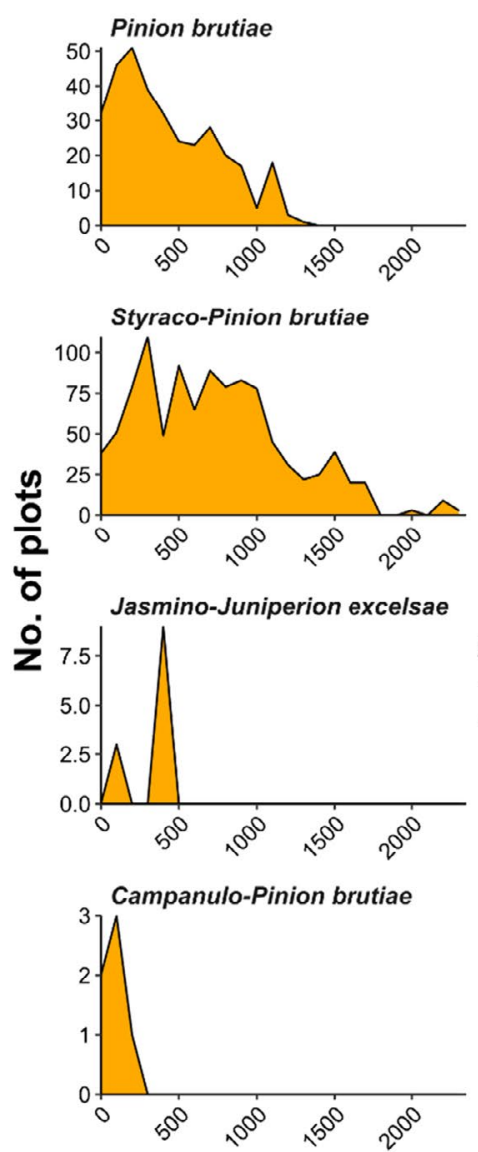
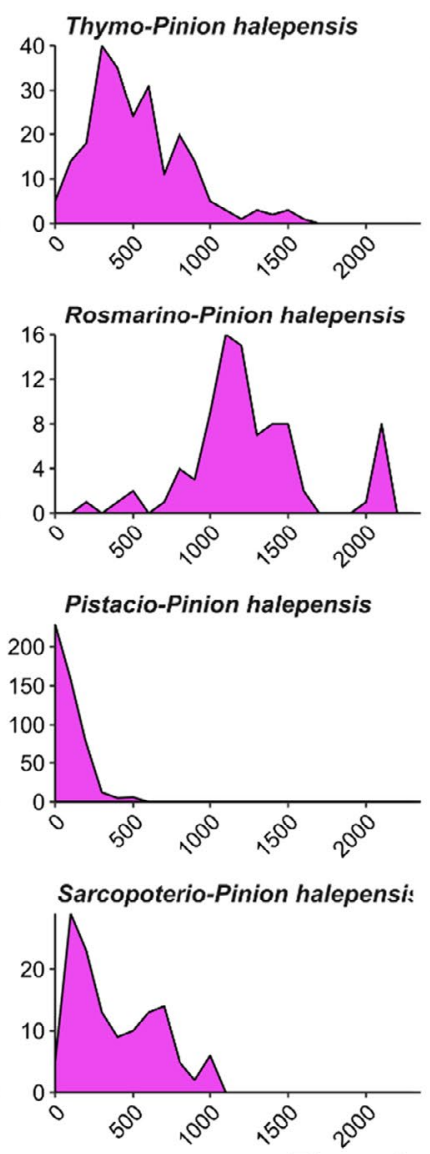
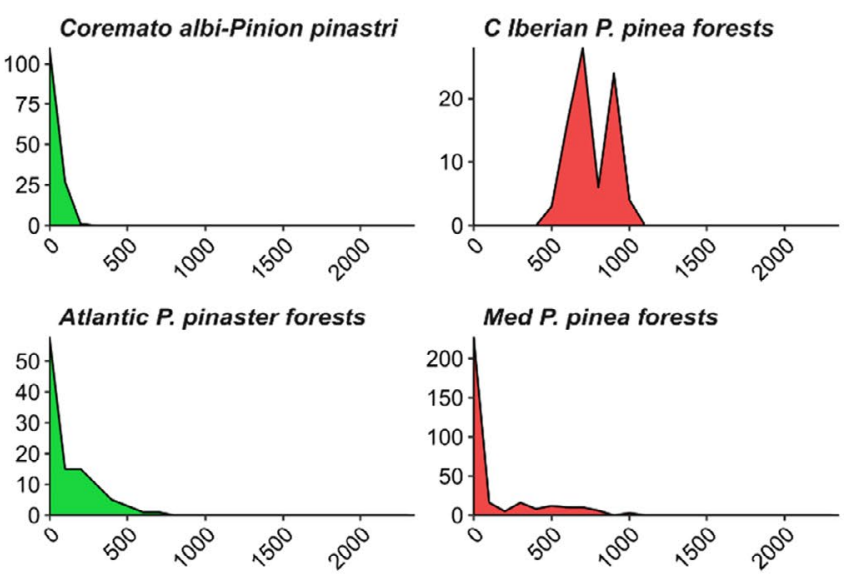

Lavandulo-Pinion pinastri
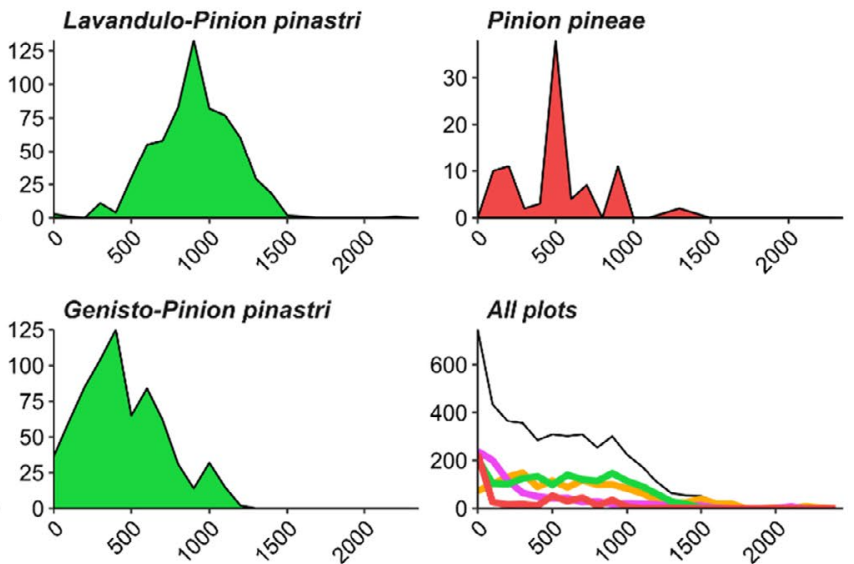

\section{Elevation (m a.s.l.)}

FIGURE 6 Elevational-density graphs for the resampled data set of vegetation plots classified by the expert system to individual alliances ( $n=4,468$; a subset of plots with an indication of elevation). Alliances dominated by individual pine species are shown in different colours. $\mathrm{C}=$ central; $\mathrm{Med}=$ Mediterranean. All plots = cumulative curve of all plots (in black) and of each pine species (orange = Pinus brutia; violet $=$ Pinus halepensis; green = Pinus pinaster; red = Pinus pinea). Full names of alliances and informal groups are reported in paragraphs 3.1.1-3.1.15 


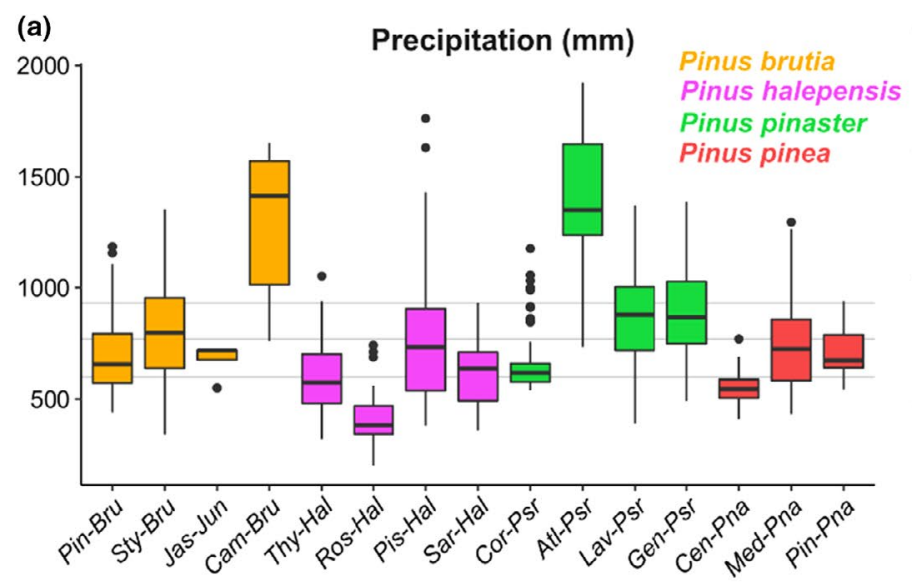

(b)

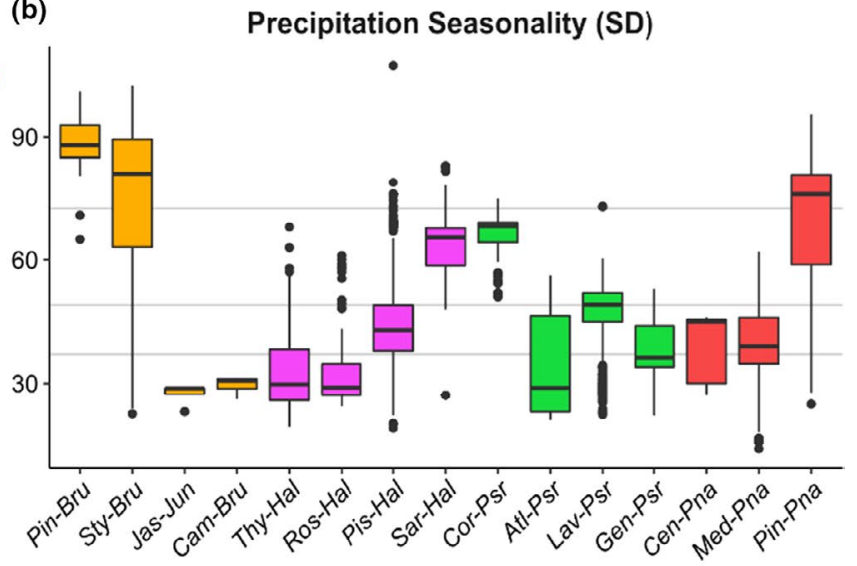

(c)

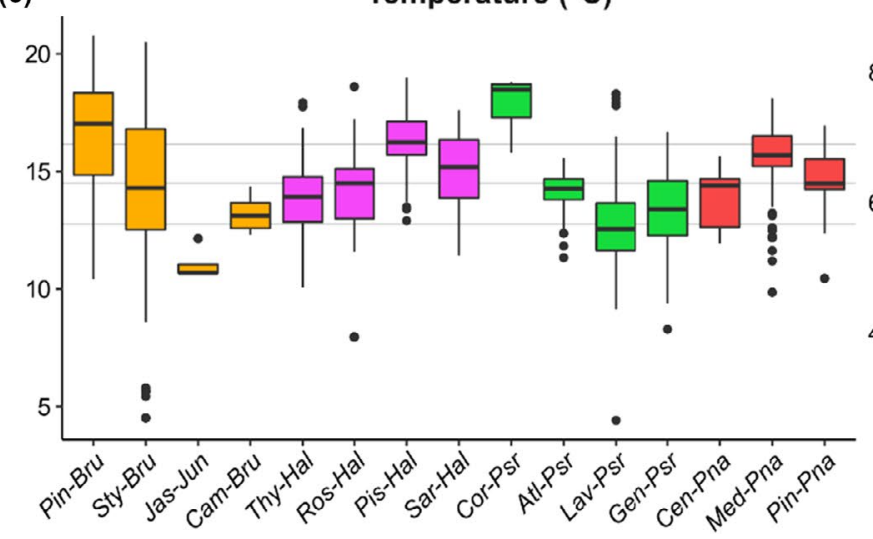

(d)

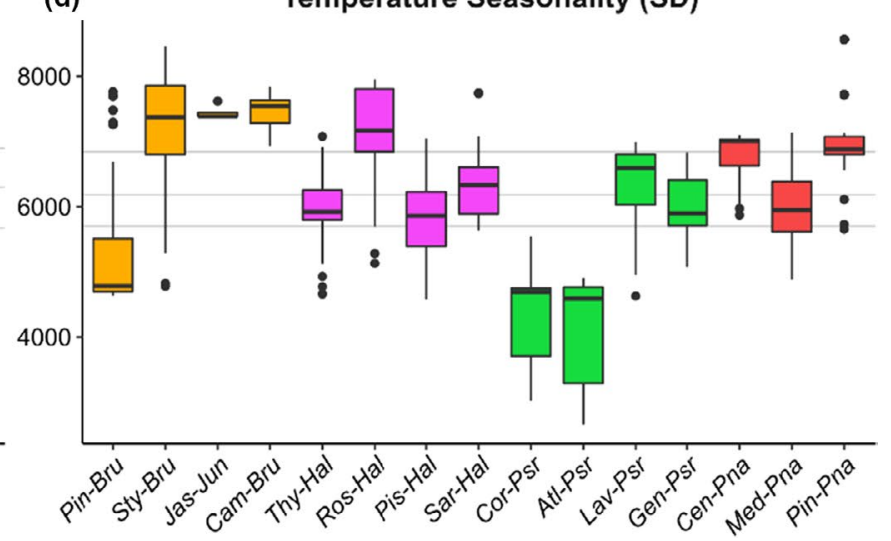

FIGURE 7 Boxplots of climatic variables for the data set of resampled vegetation plots $(n=4,468)$ classified by the expert system to individual alliances and informal groups. See paragraphs 3.1.1-3.1.15 for alliance acronyms. Grey lines represent the upper quartile, the median and the lower quartile for all plots $(n=4,468)$

\section{4 | DISCUSSION}

\section{1 | Mediterranean and non-Mediterranean pine forest alliances}

We propose some changes in the system of alliances published in EuroVegChecklist (Mucina et al., 2016) for Europe, but also for North Africa, for which we identified a new alliance of pine (pre-)forests. Following the physiognomic classification approach at the class and order level, we assigned the alliances of the vegetation dominated by Mediterranean thermophilous pine species to the order Pinetalia halepensis. We placed in this order the alliances Pinion brutiae, Styraco officinalis-Pinion brutiae, Jasmino fruticantis-Juniperion excelsae, Campanulo sibiricae-Pinion brutiae, Thymo vulgaris-Pinion halepensis, Rosmarino eriocalycis-Pinion halepensis, Pistacio lentisci-Pinion halepensis, Sarcopoterio spinosi-Pinion halepensis, Coremato albi-Pinion pinastri, Lavandulo pedunculatae-Pinion pinastri, Genisto pilosae-Pinion pinastri and Pinion pineae. We also included here three informal groups: Atlantic Pinus pinaster forests, Central Iberian Pinus pinea forests and Mediterranean Pinus pinea forests. Data analysis showed that some of the earlier described alliances are not supported, or cannot be separated. For example, the diagnostic species of the alliance Pistacio
lentisci-Pinion halepensis largely overlap with those of Rosmarino officinalis-Pinion halepensis. Therefore, we synonymized these two alliances.

The alliance Alkanno baeoticae-Pinion halepensis was described by Mucina et al. (2009) but not recognized in our study since it is documented by very few plots. It was described from a small area with ultramafic bedrock (peridotite) on the Greek island of Evvia (Euboea), but the authors of the original description were uncertain about the distribution of this alliance ("we presume its occurrence also in the adjacent Greek mainland on appropriate geological substrates"). Further studies in the field are needed to understand the syntaxonomical status of this vegetation unit.

In the EuroVegChecklist, the Crimean alliance Jasmino fruticantis-Juniperion excelsae was assigned to the Berberido creticae-Juniperetalia excelsae order of the Junipero-Pinetea sylvestris class. Based on physiognomy and species composition, it is better to accommodate Crimean Pinus brutia forests (Jasmino fruticantis-Juniperion excelsae) in Pinetalia halepensis. However, further analyses are needed to disentangle the hierarchical status of Jasmino fruticantis-Juniperion excelsae as these forests grow only at two relatively small sites mixed with sparse forests of Juniperus excelsa, which are much more widespread.

For completeness, our expert system and syntaxonomical scheme also report the alliance Campanulo sibiricae-Pinion brutiae, which 
comprises Pinus brutia-dominated forests on the south-facing slopes of the western Great Caucasus above the Black Sea near Novorossiysk (Litvinskaya \& Postarnak, 2002; Mucina et al., 2016). The species composition of these forests is close to that of the deciduous forests of the alliance Carpino orientalis-Quercion pubescentis and they were classified to the syntaxa of deciduous thermophilous oak forests in EuroVegChecklist: Quercetalia pubescenti-petraeae and Quercetea pubescentis (but see also Didukh, 1996). Although the Pinus brutia forests in southern Crimea (Jasmino fruticantis-Juniperion excelsae) also contain several species of deciduous oak forests, they harbour more Mediterranean species and structural features than their counterparts in the western Caucasus. Further studies are needed to clarify the position of the latter forests. In particular, they will need to be compared with forests of Erico-Pinetea, Brachypodio pinnati-Betuletea pendulae and Quercetea pubescentis.

\section{2 | Pinetea halepensis: a new class of the Mediterranean thermophilous pine forests}

The current European vegetation classification (Mucina et al., 2016) puts a strong emphasis on the physiognomy of the dominant layer in the definitions of vegetation classes. For example, it separates the class of temperate broad-leaved acidophilous forests (Quercetea robori-petraeae) from that of boreal to temperate coniferous forests (VaccinioPiceetea) in spite of considerable overlap in species composition, especially in Central European lowland oak and pine forests (Heinken, 2008; Leuschner \& Ellenberg, 2017). Similarly, also the non-Mediterranean southern European deciduous oak and pine forests are separated at the class level (Quercetea pubescentis vs. Erico-Pinetea). In this context, the inclusion of the Mediterranean sclerophyllous oak and pine forests in a single class Quercetea ilicis, as proposed by Mucina et al. (2016), is inconsistent, hard to convey to practitioners and difficult to apply in remote sensing of vegetation and land-cover classifications. It also has no clear links to the broadly used systems of habitats or forest types, which usually in the first place make a distinction between broadleaved and coniferous forests (Barbati et al., 2006; Chytrý et al., 2020).

With this in mind, we establish here a new class named Pinetea halepensis to accommodate the Mediterranean thermophilous pine forests addressed in this paper. This class corresponds to the EUNIS habitat type "T3A Mediterranean lowland to submontane Pinus forest," and partly also to "N1G Mediterranean coniferous coastal dune forest" (Chytrý et al., 2020). The new syntaxonomic solution, uniting all of these pine forests in one class, is justified especially by the structural and physiognomic criteria. Also ecologically, natural pine forests are united by their occurrence in either climatically or edaphically extreme environments, such as the most exposed, warm and dry rocky slopes, often on ultramafic bedrocks, marls, dolomites or limestones. This new concept is well supported by the comparative analysis of the phi coefficients for the most frequent species of the classes Pinetea halepensis and Quercetea ilicis (Table 3). Narrow-leaved xerophytes are chiefly present in Pinetea halepensis (e.g. Helichrysum stoechas, Rosmarinus officinalis, Teucrium polium aggr.), as opposed to several broad-leaved or "broad-phyllocladian" species mainly present in Quercetea ilicis (e.g. Asplenium adiantum-nigrum, Asplenium trichomanes, Dioscorea communis, Rosa sempervirens, Ruscus aculeatus). Beside showing the drier nature of the pine forest understorey, the presence of narrow-leaved xerophytes can also be tentatively interpreted as an adaptation/response of the species composition to different degrees of insolation between the open (Pinetea halepensis) vs. closed canopy (Quercetea ilicis), a further
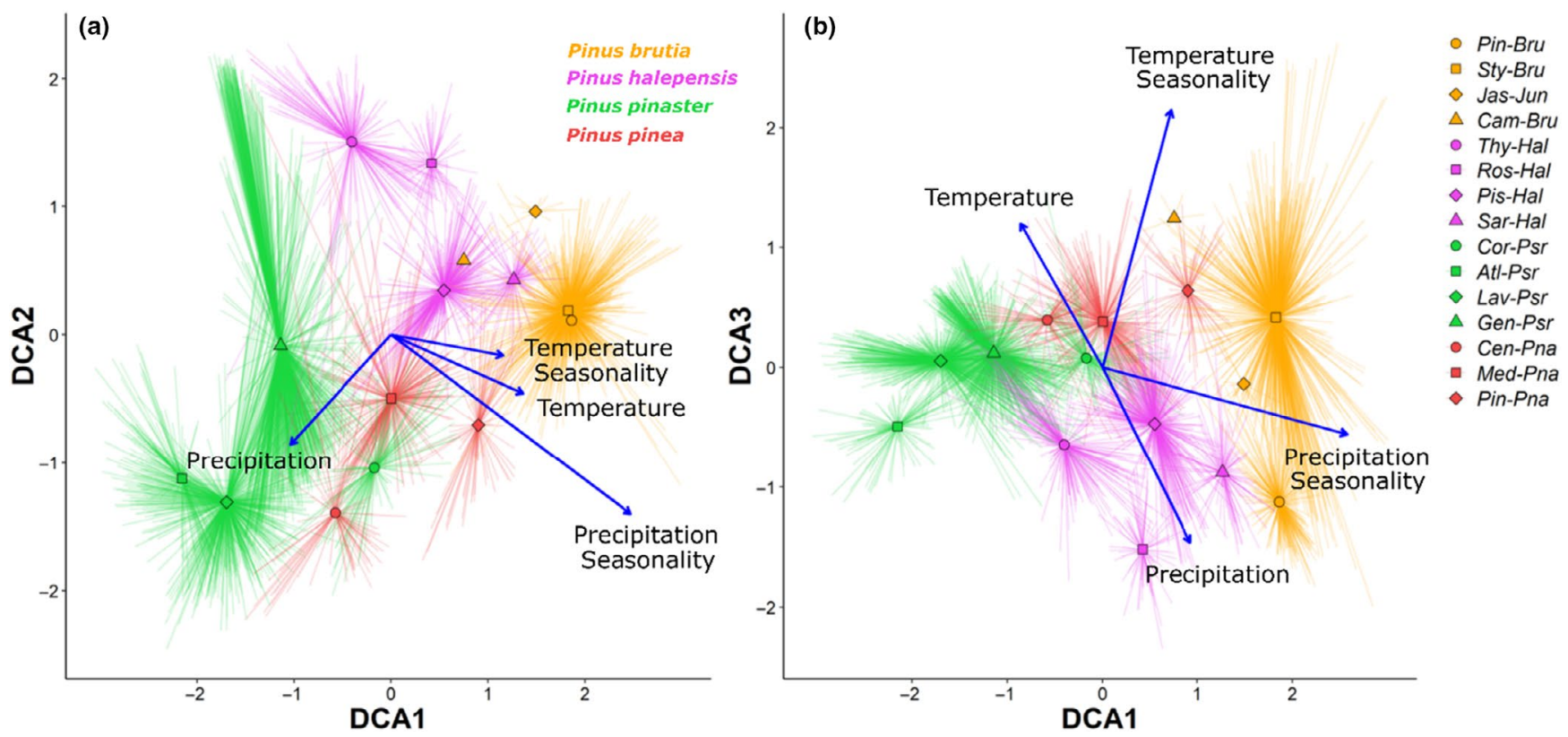

FIGURE 8 DCA ordinations ( $A=$ axes 1-2; $B=$ axes 1-3) of the resampled data set of vegetation plots $(n=4,468)$ classified by the expert system to individual alliances/informal group. Plots are grouped by alliances, with tips of the lines connecting the positions of individual vegetation plots with alliance centroids. The blue arrows represent climatic variables. See paragraphs 3.1.1-3.1.15 for acronyms 
TAB LE 4 The new syntaxonomical scheme for low-elevation Mediterranean pine forests

\section{Syntaxonomic scheme}

Pinetea halepensis Bonari et Chytrý in Bonari et al. 2021

Pinetalia halepensis Biondi, Blasi, Galdenzi, Pesaresi et Vagge in Biondi et al. 2014

- Pinion brutiae Feinbrun 1959

- Styraco officinalis-Pinion brutiae Bonari, Chytrý, Çoban, Kavgacı et Sağlam in Bonari et al. 2021 (New)

- Jasmino fruticantis-Juniperion excelsae Didukh, Vakarenko et Shelyag-Sosonko ex Bonari et al. 2021 (EVC/More research/ Validated)

- Thymo vulgaris-Pinion halepensis Biondi et Pesaresi in Pesaresi et al. 2017

- Rosmarino eriocalycis-Pinion halepensis Bonari, Chytrý et Fernández-González in Bonari et al. 2021 (New)

o Pistacio lentisci-Pinion halepensis Biondi, Blasi, Galdenzi, Pesaresi et Vagge in Biondi et al. 2014 (EVC)

o Sarcopoterio spinosi-Pinion halepensis Biondi et Pesaresi in Pesaresi et al. 2017

- Alkanno baeoticae-Pinion halepensis Mucina et Dimopoulos in Mucina et al. 2009 (EVC/More research)

- Coremato albi-Pinion pinastri J.C. Costa, Neto, Capelo, Aguiar, Monteiro-Henriques et Bonari in Bonari et al. 2021 (New)

- Atlantic Pinus pinaster forests (Informal)

- Lavandulo pedunculatae-Pinion pinastri Fernández-González, Bonari et Chytrý in Bonari et al. 2021 (New)

- Genisto pilosae-Pinion pinastri Biondi et Vagge 2015 (EVC)

$\diamond$ Central Iberian Pinus pinea forests (Informal)

$\diamond$ Mediterranean Pinus pinea forests (Informal)

$\diamond$ Pinion pineae Feinbrun 1959 (EVC)

Quercetea pubescentis Doing-Kraft ex Scamoni et Passarge 1959

Quercetalia pubescenti-petraeae Klika 1933 corr.

- Campanulo sibiricae-Pinion brutiae Litvinskaya et Postarnak ex Mucina in Mucina et al. 2016 (EVC/More research/Unresolved)

Units at syntaxonomical levels lower than order are sorted by the dominance of different pine species that is indicated by a solid circle (Pinus brutia), empty circle (Pinus halepensis), solid diamond (Pinus pinaster) and empty diamond (Pinus pinea). Abbreviations in parentheses add information for each given alliance or informal group. EVC = alliance present in the EuroVegChecklist (Mucina et al., 2016); Informal = informal group; More research = more research is needed for the alliance; New = alliance newly described in this paper; Unresolved = the syntaxonomical position of the syntaxon is unresolved; Validated $=$ alliance validated in this paper.

difference between the two classes. The observation that pine forests of the Mediterranean Basin are confined to specific edaphic conditions under which oaks do not develop into canopy dominants or are entirely absent is not novel (see e.g. Feinbrun, 1959). In some areas such as Anatolia, there is no evidence of vegetation development towards sclerophyllous oak formations in Pinus brutia forests (Akman et al., 1978; Quézel, 1986). Another example of no evidence of succession towards sclerophyllous oak forests is also found in arid areas with annual precipitation below $350 \mathrm{~mm}$ in southeastern Spain, in the lower Ebro valley and other scattered spots along the Mediterranean coast. The only tree species able to grow in these areas is Pinus halepensis (except for the very localized Tetraclinis articulata and Juniperus thurifera formations). Nevertheless, in the more natural landscapes of these semiarid areas, sclerophyllous shrublands are more common than Pinus halepensis forests.

Support for the concept of this new class also comes from palaeopalynology, palaeoanthracology and archaeology. Pinus pinaster has been shown to dominate a well-developed forest type on the Iberian Peninsula at certain time windows in the period between 31,000 and $3,000{ }^{14} \mathrm{C}$ year BP (Carrión et al., 2000 and references therein). Also, the presence of Pinus pinaster on the coastal dunes of Portugal has been reported during the first half of the Holocene (Mateus \& Queiroz, 1993; García-Amorena et al., 2007), but also charcoal remains dating from 33,000 years BP were found in the Lisbon region, making up 93\% of all the remains present (Figueiral, 1993). Similarly, there is evidence about the native status of Pinus pinea on the Iberian Peninsula, both on the southern coast (from 40,000 years ago to pre-Roman times) and in central Spain (northern Castilian plateau, 2,600-2,500 years BP, i.e. pre-Roman time). The problem with this species is that its pollen cannot always be differentiated morphologically from the pollen of the widespread Pinus pinaster, therefore, macrofossils (pine nuts, cones, wood) are needed to confirm the presence of Pinus pinea in old deposits. The existence of the low-elevation Mediterranean pine forests for millennia is an argument in support of considering them as a separate vegetation unit of high hierarchical rank.

The nomenclatural type of this class is the order Pinetalia halepensis Biondi, Blasi, Galdenzi, Pesaresi et Vagge in Biondi et al. 2014, previously included in the class Quercetea ilicis. Its most important diagnostic feature is the dominance of one of the four low-elevation Mediterranean pines. The character species of the new class (based on Biondi et al., 2014; Pesaresi et al., 2017 and the results of the present study) include Pinus brutia, Pinus halepensis, Pinus pinaster and Pinus pinea, while many typical Mediterranean woody species such as Arbutus unedo, Asparagus acutifolius, Ceratonia siliqua, Cistus creticus, Cistus salviifolius, Erica arborea, Juniperus oxycedrus, Myrtus communis, Olea europaea, Phillyrea latifolia, Pistacia lentiscus, Pistacia terebinthus, Rhamnus alaternus, Rubia peregrina and Smilax aspera are joint character species of the Pinetea halepensis and other classes of Mediterranean vegetation, mainly Quercetea ilicis but also CistoLavanduletea stoechadis and Ononido-Rosmarinetea. This reflects the very close floristic and often dynamic relationship between many of the pine forests analysed in this study and communities of broadleaved sclerophyllous woodlands and shrublands of macchia and garrigue.

Mediterranean thermophilous pine forests have recently undergone profound classification reinterpretations at the order level. Pinus halepensis communities have been traditionally considered as a part of the order of Mediterranean sclerophyllous scrub (macchia, maquis), Pistacio lentisci-Rhamnetalia alaterni (e.g. RivasMartínez et al., 1986) or evergreen Mediterranean oak forests and macchia, Quercetalia ilicis (e.g. Horvat et al., 1974; Rodwell et al., 2002). Recently, a new order of the Mediterranean thermophilous pine forests was established by Biondi et al. (2014). These authors 
defined it as native forests of Pinus halepensis and Pinus pinea of the Mediterranean, including old-established plantations present within their native distribution range. Besides the diagnostic species reported by Biondi et al. (2014; i.e. Erica arborea, Juniperus oxycedrus, Juniperus phoenicea subsp. turbinata, Myrtus communis, Pinus halepensis, Pinus pinea, Pistacia lentiscus, Rosmarinus officinalis), here we also add Pinus brutia and Pinus pinaster and extend the original definition of this order by including also native and old-established plantations dominated by the four low-elevation Mediterranean pines.

\section{3 | Old-established coastal pine plantations: to be or not be (a phytosociological unit)?}

Mediterranean pine forests have not traditionally received much attention from phytosociologists, because pines have been planted throughout the Mediterranean Basin for millennia (Bonari et al., 2017). Although most plantations were established in the 20th century (especially on the Iberian Peninsula), in many cases, it is challenging to trace whether or not a pine forest is natural.

Our classification includes informal vegetation types comprising old-established plantations of native pine species, in which natural species composition can develop in the understorey (Bonari et al., 2017, 2019a, 2020). This is in contrast to what happens in the plantations of most non-native trees (e.g. Eucalyptus spp.). The old pine plantations then resemble natural pine forests, but establishing syntaxonomical units for them would deviate from the tradition of the phytosociological classification of forests, which is focused on natural forests or at least on the vegetation dominated by spontaneously-established native trees. For these reasons, we do not establish formal syntaxa for pine plantations in habitats and areas where particular pines are considered non-native or plantations are likely more common than native communities. Still, we distinguish informal units including old-established plantations, because they were recognized in the unsupervised classification in our study and have, to some extent, a distinct species composition. These units may also include some supposedly native forests. Beside the localities where they were undoubtedly planted, e.g. along the Italian Peninsula coast, especially Pinus pinea communities require further research at the local scale using palaeobotanical evidence, old written documents and other sources, to assess the origin of each population. Irrespective of their origin, these forests largely correspond to the priority habitat 2270 "Wooded dunes with Pinus pinea and/or Pinus pinaster" of Annex I of the EU Habitats Directive (92/43/EEC) and to "T3A Mediterranean lowland to submontane Pinus forest" and "N1G Mediterranean coniferous coastal dune forest" in the EUNIS Habitat Classification (Chytrý et al., 2020) and they are considered valuable for nature conservation (Bonari et al., 2018).

\section{4 | Data limitations and recommendations for future vegetation surveys}

We have laid down a classification which does not pretend to be perfect. We are aware of the fact that some areas were underrepresented in our analyses because of the lower density of plots, meaning that we might have overlooked some vegetation types or some diagnostic species. Nevertheless, a clear advantage of our classification is that it is formally described and reproducible. Also, by covering the full distribution range of the studied dominant pine species, we encompass the full species pool of these forests, so that the alliances can acquire a relevant biogeographical meaning. The expert system (Appendix S2) contains groups of species and decision rules that enable identification of each of the pine forest alliances recognized here.

Some pine-dominated vegetation plots remained unclassified after running the expert system. For example, a considerable proportion of Pinus halepensis-dominated plots, equally distributed around the Mediterranean Basin, remained unclassified at the alliance level, although they were correctly classified at the class and order level. Because of the open-canopy structure of pine forests, which allows the occurrence of species from various habitats, these plots contained a mixture of species with different ecology. Nevertheless, our expert system showed that there is a large portion of plots with Pinus halepensis that can be classified. The unclassified plots are more or less equally distributed across the study area, which points to local-scale effects (including disturbance such as fire, forestry management or trampling) that make the classification of Mediterranean pine forests challenging.

Further, some areas in our data set are represented by very species-poor plots, in some cases with one to three species only (e.g. in the Levant). Such plots are problematic because they were perhaps sampled in very disturbed areas, but sometimes they were the only data available from a broader area. Disturbances may have influenced the classification results. For instance, if vegetation plots in biogeographically contrasting areas experienced more intensive forestry management (plantation, re/afforestation, timber extraction), they can become species-poor and the overall effect is weakening of the biogeographical patterns in the classification. This fact contrasts with clearer biogeographical patterns of other alliances identified in this study. Also, the open canopy allows the entry of many generalist (e.g. ruderal and annual) species. We suggest that with the increasing availability of large vegetation-plot databases and detailed revisions of vegetation classification, broadly conceived geographically defined alliances will be delineated more often than in the past.

Other Mediterranean conifer forests dominated by Juniperus, Cupressus, or Tetraclinis share some structural aspects with pine forests (e.g. relatively open canopies, litter decomposition), but in general, have not been managed so heavily. Some of them reach heights much lower than the pines, but others can be comparable in height when the forest is undisturbed. Their right position in the syntaxonomical scheme of Mediterranean forests should be revised through a large-scale analysis.

Collecting new data in scarcely surveyed areas is needed in the future. In particular, for Pinus brutia, we miss data from the easternmost limit of its distribution (Azerbaijan, Armenia, Iran and Iraq) and also from Israel. For Pinus halepensis, we miss data from northern Libya and Albania. For Pinus pinaster, we miss mainly data from North African countries. For Pinus pinea, we miss data from Southern 
Balkans and some Mediterranean islands. These new data could provide evidence for recognizing new syntaxa or reinterpreting and redefining the earlier proposed vegetation units.

Despite the Food and Agriculture Organization (FAO) and most of the national forest inventories usually using $20 \%$ canopy cover as a threshold for forest, we used a cover of $15 \%$. We advocate that the traditional Braun-Blanquet cover value 2, including covers of $5 \%-25 \%$, is relatively broad for our purposes and does not ensure by itself that a given plot belongs to a "forest" or shrubland with a few pines. Therefore, the decision to use $15 \%$ represents a compromise for not excluding too many pine (pre-)forests plots with an open canopy. For such large-scale analyses, old sampled plots are also crucial, and we used them. However, for sampling new plots, we recommend that at least a cover value of 3 should apply when selecting plot areas for pine forests. Another related problem is that in many old plots the growth form (either shrub or tree) is not indicated for woody plants. Therefore, we also recommend an indication of the height of the strata to recognize the forest structure properly and to evaluate whether or not pines are the dominant trees.

\section{5 | CONCLUSIONS}

We propose a new syntaxonomical scheme for the low-elevation Mediterranean pine forests (Table 4) with 15 alliances (or informal groups), four of them described as new. Generally, each alliance is dominated by one of the Mediterranean thermophilous pine species (Pinus brutia, Pinus halepensis, Pinus pinaster, Pinus pinea) and is largely related to specific elevational belts (thermo-, meso- or supramediterranean) and a position on the west-east, and partly northsouth, biogeographical gradients.

Our broad-scale classification of the Mediterranean thermophilous pine forests distinguished and documented 12 alliances of native forests and three informal groups. Conceptual considerations have allowed us to include the recognized units into a newly-established class (Pinetea halepensis) and its subordinate order (Pinetalia halepensis).

In comparison with EuroVegChecklist (Mucina et al., 2016), this study has enriched the syntaxonomical system of Europe by four newly recognized alliances (Coremato albi-Pinion pinastri, Lavandulo pedunculatae-Pinion pinastri, Styraco officinalis-Pinion brutiae) and one newly recognized alliance for North Africa (Rosmarino eriocalycis-Pinion halepensis). One previously invalidly described alliance was validated (Jasmino-Juniperion excelsae). In contrast, a previously described alliance (Rosmarino officinalis-Pinion halepensis) was not supported by the analysis of a large data set. The alliances Alkanno baeoticae-Pinion halepensis, Campanulo sibiricae-Pinion brutiae and Jasmino fruticantis-Juniperion excelsae with restricted geographic distribution will require further research.

The results of this study provide information on the compositional and distributional patterns of Mediterranean thermophilous pine forests, offering a list of statistically derived combinations of diagnostic species for the major eco-geographical vegetation units. Further, the workflow adopted in this study, but also its pitfalls and limitations, might be useful as a pathway for similar broad-scale vegetation classification studies.

This classification study sheds light on the biodiversity, biogeography and environment of the Mediterranean thermophilous pine forests. It complements the existing systems of habitat classification used for nature conservation such as the EUNIS Habitat Classification and the EU Habitats Directive, thus providing a tool for better conservation planning, monitoring and management at both the international and national level.

\section{ACKNOWLEDGEMENTS}

We thank the participants of the IAVS Symposia in Bozeman, Montana, USA and Bremen, Germany and of the European Vegetation Survey meetings in Wrocław, Poland and Madrid, Spain for valuable discussions that helped improve this paper. We are further indebted to Jorge Capelo, Xavier Font Castell and Simone Pesaresi for their comments on a previous version of this manuscript, Borja Jiménez-Alfaro for the SIVIM reference list, Anna Kuzemko and Denys Vynokurov for Russian and Ukrainian translations, Ondřej Hájek for preparing the climatic data for the former version of this manuscript, Petr Šmarda for helping us with taxonomic interpretation of Festuca records, Daniela Gigante, Riccardo Guarino, Corrado Marcenò and Pavel Novák for stimulating discussions, Olivier Argagnon, Mikhail Beskaravayny, Adil Çalışkan and Jalal El Oualidi for photographs of some pine forest types, Mohamed Fennane for providing contacts for photographic material, Lamia Bahbah, Rachda Berrached and Agnese Tilia for providing some missing literature, custodians of the EVA vegetation databases, namely Iva Apostolova, Idoia Biurrun, Henry Brisse, Juan Antonio Campos, Andraž Čarni, János Csiky, Mirjana Ćuk, Els De Bie, Iris De Ronde, Michele De Sanctis, Panayotis Dimopoulos, Laura Casella, Renata Ćušterevska, Xavier Font, Emmanuel Garbolino, Rosario Gavilán, JeanClaude Gégout, Friedemann Goral, Adrian Indreica, Ute Jandt, Florian Jansen, Borja Jiménez-Alfaro, Zygmunt Kącki, Vladimir Onipchenko, Viktor Onyshchenko, Tomáš Peterka, Remigiusz Pielech, Eszter Ruprecht, Urban Šilc, Zvjezdana Stančić, Ioannis Tsiripidis, Emin Uğurlu, Milan Valachovič, Kiril Vassilev, Roberto Venanzoni, Wolfgang Willner, Thomas Wohlgemuth and contributors of the CircumMed Pine Forest Vegetation Database for making their plots available. Last but not least, we thank all the botanists and vegetation ecologists who collected the pine forest data in the field, thus making this synthesis possible.

\section{AUTHOR CONTRIBUTIONS}

GB and MC conceived the idea; GB, IK and MC developed the database; $\mathrm{GB}$ conducted the analysis, under the supervision of $\mathrm{MC}$; $\mathrm{SMH}$ and LT provided support for data processing in TURBOVEG 3 and JUICE; JD helped with the interpretation of plant names; KC prepared maps and graphs; GB led the writing, with substantial inputs from MC; all the co-authors participated in discussions and syntaxonomic interpretations. 


\section{DATA AVAILABILITY STATEMENT}

Vegetation-plot data used in this study are available in the CircumMed Pine Forest Vegetation Database (GIVD ID: EU-00-026) and European Vegetation Archive (EVA). They can be obtained upon request from the EVA database administrator with reference to EVA project no. 57.

\section{ORCID}

Gianmaria Bonari iD https://orcid.org/0000-0002-5574-6067 Federico Fernández-González (iD) https://orcid. org/0000-0003-1234-4065 Süleyman Çoban (D) https://orcid.org/0000-0003-1570-9795 Tiago Monteiro-Henriques (iD https://orcid. org/0000-0002-4206-0699 Erwin Bergmeier iD https://orcid.org/0000-0002-6118-4611 Yakiv P. Didukh iD https://orcid.org/0000-0001-7619-0283 Fotios Xystrakis (iD https://orcid.org/0000-0002-3318-2686 Claudia Angiolini (D) https://orcid.org/0000-0002-9054-9480 Kryštof Chytrý iD https://orcid.org/0000-0003-4113-6564 Alicia T.R. Acosta iD https://orcid.org/0000-0001-6572-3187 Emiliano Agrillo iD https://orcid.org/0000-0003-2346-8346 José C. Costa (iD https://orcid.org/0000-0002-7619-840X Jiří Danihelka iD https://orcid.org/0000-0002-2640-7867 Stephan M. Hennekens (D) https://orcid.org/0000-0003-1221-0323 Ali Kavgacl iD https://orcid.org/0000-0002-4549-3668 Ilona Knollová iD https://orcid.org/0000-0003-4074-789X Carlos S. Neto iD https://orcid.org/0000-0003-0912-0255 Coşkun Sağlam (iD https://orcid.org/0000-0003-2980-2501 Željko Škvorc (iD https://orcid.org/0000-0002-2848-1454 Lubomír Tichý (iD https://orcid.org/0000-0001-8400-7741 Milan Chytrý (iD https://orcid.org/0000-0002-8122-3075

\section{REFERENCES}

Abad Viñas, R., Caudullo, G., Oliveira, G. and de Rigo, D. (2016a) Pinus pinaster in Europe: Distribution, habitat, usage and threats. In: SanMiguel-Ayanz, J., de Rigo, D., Caudullo, G., Houston Durrant, T. and Mauri, A. (Eds), European Atlas of Forest Tree Species. Luxembourg, LU: Publications Office of the European Union, p. e012d59+.

Abad Viñas, R., Caudullo, G., Oliveira, G. and de Rigo, D. (2016b) Pinus pinea in Europe: Distribution, habitat, usage and threats. In: SanMiguel-Ayanz, J., de Rigo, D., Caudullo, G., Houston Durrant, T. and Mauri, A. (Eds), European Atlas of Forest Tree Species. Luxembourg, LU: Publications Office of the European Union, p. e01b4fc+.

Abi-Saleh, B., Barbéro, M., Nahal, I. and Quézel, P. (1976) Les séries forestières de végétation au Liban. Essai d'interprétation schématique. Bulletin de La Société Botanique de France, 123(9), 541-560.

Akman, Y., Barbéro, M. and Quézel, P. (1978) Contribution à l'étude de la végétation forestière d'Anatolie méditerranéenne. Phytocoenologia, 5(1), 1-79.

Akman, Y., Barbéro, M. and Quézel, P. (1979) Contribution à l'étude de la végétation forestière d'Anatolie méditerranéenne. Phytocoenologia, 5(3), 277-346.

Akman, Y., Kurt, L., Demiryürek, E., Quézel, P., Kurt, F., Evren, H. et al (1998) Les groupements à Pinus brutia sur roches ultra-basiques et calcaires, dans la région de Marmaris et Bodrum (Mugla), à l'étage thermo-méditerranéen du sud-ouest. Ecologia Mediterranea, 24(1), 63-71.

Akman, Y., Yurdakulol, E. and Demirörs, M. (1983) The vegetation of the Ilgaz Mountains. Ecologia Mediterranea, 9(2), 137-165.
Arrigoni, P.V. (1967) Ricerche sulla distribuzione del Pinus halepensis Mill. e del Pinus pinea L. in Sardegna. Webbia, 22(2), 405-418.

Barbati, A., Corona, P. and Marchetti, M. (2006) European forest types. Categories and types for sustainable forest management and reporting. European Environment Agency, EEA Technical report No. 9/2006, ISSN 1725-2237.

Barbéro, M., Chalabi, N., Nahal, I. and Quézel, P. (1976) Les formations à conifères méditerranéens en Syrie littorale. Ecologia Mediterranea, 2, 87-99.

Barbéro, M., Loisel, R. and Quézel, P. (1974) Problèmes posés par l'interprétation phytosociologique des Quercetea ilicis et des Quercetea pubescentis. Colloques Internationaux du C.N.R.S., 235, 481-497.

Barbéro, M., Loisel, R., Quézel, P., Richardson, D.M. and Romane, F. (1998) Pines of the Mediterranean Basin. In: Richardson, D.M. (Ed.) Ecology and Biogeography of Pinus. Cambridge, UK: Cambridge University Press, pp. 153-170.

Barbéro, M. and Quézel, P. (1976) Les groupements forestiers de Grèce centro-méridionale. Ecologia Mediterranea, 2, 3-85.

Barbéro, M. and Quézel, P. (1979) Contribution à l'étude des groupements forestiers de Chypre. Documents Phytosociologiques, 4(1), 9-34.

Barbéro, M., Quézel, P. and Rivas-Martínez, S. (1981) Contribution à l'étude des groupements forestiers et préforestiers du Maroc. Phytocoenologia, 9(3), 311-412.

Benabid, A. (1988) Contribution à l'étude des groupements non forestiers du Moyen Atlas central (Maroc). Bulletin de I'Institut Scientifique Rabat, 12, 79-98.

Biondi, E., Allegrezza, M., Casavecchia, S., Galdenzi, D., Gasparri, R., Pesaresi, S. et al (2014) New and validated syntaxa for the checklist of Italian vegetation. Plant Biosystems, 148(2), 318-332. https://doi. org/10.1080/11263504.2014.892907

Biondi, E. and Vagge, I. (2015) The forests of Pinus pinaster Aiton subsp. pinaster of the NW-Italian Tyrrhenian sector. Acta Botanica Gallica, 162(3), 239-250. https://doi.org/10.1080/12538 078.2015.1063451

Biurrun, I., Bergmeier, E., Dengler, J., Jansen, F. and Willner, W. (2019) Vegetation classification and its application are relevant globally. Phytocoenologia, 49(1), 1-6. https://doi.org/10.1127/phyto/ 2019/0323

Blondel, J., Aronson, J., Bodiou, J.-Y. and Boeuf, G. (Eds) (2010) The Mediterranean Region: Biological Diversity in Space and Time, 2nd edn. Oxford, UK: Oxford University Press.

Bonari, G., Acosta, A.T.R. and Angiolini, C. (2017) Mediterranean coastal pine forest stands: Understorey distinctiveness or not? Forest Ecology and Management, 391, 19-28. https://doi.org/10.1016/j. foreco.2017.02.002

Bonari, G., Acosta, A.T.R. and Angiolini, C. (2018) EU priority habitats: Rethinking Mediterranean coastal pine forests. Rendiconti Lincei. Scienze Fisiche e Naturali, 29(2), 295-307. https://doi.org/10.1007/ s12210-018-0684-9

Bonari, G., Chytrý, K., Çoban, S. and Chytrý, M. (2020) Natural forests of Pinus pinea in western Turkey: a priority for conservation. Biodiversity and Conservation, 29, 3877-3898. https://doi.org/10.1007/s1053 1-020-02052-z

Bonari, G., Knollová, I., Vlčková, P., Xystrakis, F., Çoban, S., Sağlam, C. et al (2019b) CircumMed Pine Forest Database: An electronic archive for Mediterranean and Submediterranean pine forest vegetation data. Phytocoenologia, 49(3), 311-318. https://doi.org/10.1127/ phyto/2019/0311

Bonari, G., Těšitel, J., Migliorini, M., Angiolini, C., Protano, G., Nannoni, F. et al (2019a) Conservation of the Mediterranean coastal pine woodlands: How can management support biodiversity? Forest Ecology and Management, 443, 28-35. https://doi.org/10.1016/j. foreco.2019.04.005

Boydak, M., Dirik, H. and Çalıkoğlu, M. (2006) Biology and silviculture of Turkish Red Pine (Pinus brutia Ten.), Ankara, TR: OGEM-VAK yayını. 
Braun-Blanquet, J. (Ed.) (1932) Plant Sociology. The Study of Plant Communities, 1st edn. New York, NY: McGraw-Hill.

Benabdeli, K. (1996) Mise en évidence de l'importance des formations basses dans la sauvegarde des écosystèmes forestiers: cas des monts de Dhaya (Algérie occidentale). Ecologia Mediterranea, 22(3-4), 101-112.

Bruelheide, H. (1997) Using formal logic to classify vegetation. Folia Geobotanica, 32(1), 41-46. https://doi.org/10.1007/BF02803883

Bruelheide, H., Dengler, J., Jiménez-Alfaro, B., Purschke, O., Hennekens, S.M., Chytrý, M. et al (2019) sPlot: a new tool for global vegetation analyses. Journal of Vegetation Science, 30(2), 181-186. https://doi. org/10.1111/jvs.12710

Brullo, S., Gianguzzi, L., La Mantia, A. and Siracusa, G. (2008) La classe Quercetea ilicis in Sicilia. Bollettino Accademia Gioenia di Scienze Naturali Catania, 41(369), 1-80.

Boydak, M. (2004) Silvicultural characteristics and natural regeneration of Pinus brutia Ten. - a review. Plant Ecology, 171, 153-163.

Cabezudo, B., Nieto-Caldera, J.M. and Pérez-Latorre, A. (1989)Contribución al conocimiento de la vegetación edafófilo-serpentinícola del sector Rondeño (Málaga; España). Acta Botanica Malacitana, 14, 291-294.

Carrión, J.S., Navarro, C., Navarro, J. and Munuera, M. (2000) The distribution of cluster pine (Pinus pinaster) in Spain as derived from palaeoecological data: Relationships with phytosociological classification. The Holocene, 10(2), 243-252. https://doi.org/10.1191/095968300676937462

Carrión Marco, Y. (2005) La vegetación mediterránea y atlántica de la península Ibérica: nuevas secuencias antracológicas. Serie de trabajos varios 104. Valencia: Servicio de Investigación Prehistórica, Diputación Provincial de Valencia.

Caudullo, G., Welk, E. and San-Miguel-Ayanz, J. (2017) Chorological maps for the main European woody species. Data in Brief, 12, 662666. https://doi.org/10.1016/j.dib.2017.05.007

Chouchani, B., Khouzami, M. and Quézel, P. (1974) A propos de quelques groupements forestiers du Liban. Ecologia Mediterranea, 1, 63-77.

Chytrý, M., Hennekens, S.M., Jiménez-Alfaro, B., Knollová, I., Dengler, J., Jansen, F. et al (2016) European Vegetation Archive (EVA): An integrated database of European vegetation plots. Applied Vegetation Science, 19(1), 173-180. https://doi.org/10.1111/avsc.12191

Chytrý, M., Tichý, L., Hennekens, S.M., Knollová, I., Janssen, F., Rodwell, J.S. et al (2020) EUNIS Habitat Classification: expert system, characteristic species combinations and distribution maps of European habitats. Applied Vegetation Science, 23(4), 648-675. https://doi. org/10.1111/avsc.12519

Çoban, S. and Willner, W. (2019) Numerical classification of the forest vegetation in the Western Euxine Region of Turkey. Phytocoenologia, 49(1), 71-106. https://doi.org/10.1127/phyto/2018/0274

Dengler, J., Janssen, F., Glöckler, F., Peet, R.K., De Cáceres, M., Chytrý, M. et al (2011) The Global Index of Vegetation-Plot Databases (GIVD): a new resource for vegetation science. Journal of Vegetation Science, 22, 582-597.

De Cáceres, M., Chytrý, M., Agrillo, E., Attorre, F., Botta-Dukát, Z., Capelo, J. et al. (2015) A comparative framework for broad-scale plot-based vegetation classification. Applied Vegetation Science, 18(4), 543-560. https://doi.org/10.1111/avsc.12179

De Marco, G., Veri, L. and Caneva, G. (1984) Analisi fitosociologica, cartografia della vegetazione e trasformazioni ambientali nel periodo 1955-1981 delle Isole Tremiti (Adriatico centro-meridionale). Annali di Botanica, 42, 17-47.

Didukh, Y.P. (Ed.) (1992) Rastitel'nyi pokrov Gornogo Kryma (struktura, dinamika, evolyutsiya i okhrana). [Vegetation cover of the Crimean Mountains (structure, dynamics, evolution and protection)]. Kyiv, UA: Naukova dumka. [In Russian.].

Didukh, Y.P., Vakarenko, L.P. and Shelyag-Sosonko, Y.R. (1986) Floristicheskaya klassifikatsiya khvoynykh lesov nizhnego poyasa rastitelnosti Gornogo Kryma [Floristic classification of coniferous forests of the lower vegetation belt of the mountainous Crimea]. Botanical Journal, 71(3), 281-291 [In Russian.].
Didukh, Y.P. (1996) The communities of the class Quercetea pubescenti-petraeae of the Crimean Mountains. Ukrainian Phytocoenological Collection, Ser. A, 1, 63-77.

Douda, J., Boublík, K., Slezák, M., Biurrun, I., Nociar, J., Havrdová, A. et al (2016) Vegetation classification and biogeography of European floodplain forests and alder carrs. Applied Vegetation Science, 19(1), 147-163. https://doi.org/10.1111/avsc.12201

Euro+Med (2016-2020) Euro+Med PlantBase - the information resource for Euro-Mediterranean plant diversity. Available at http://ww2.bgbm. org/EuroPlusMed/query.asp [Accessed 1 June 2019]

Farjon, A. and Filer, D. (Eds) (2013) An Atlas of the World's Conifers: An Analysis of Their Distribution, Biogeography, Diversity, and Conservation Status. Leiden, NL: Brill.

Feinbrun, N. (1959) Spontaneous pineta in the Lebanon. Bulletin of the Research Council of Israel, 7, 132-153.

Fennane, M. (2003) Inventaire des communautés végétales à l'aide du phytosociologue, au Maroc. Ecologia Mediterranea, 29(1), 87-106.

Fernandes, P.M. and Rigolot, E. (2007) The fire ecology and management of maritime pine (Pinus pinaster Ait.). Forest Ecology and Management, 241(1-3), 1-13. https://doi.org/10.1016/j.foreco.2007.01.010

Figueiral, I. (1993) Cabeço de Porto Marinho: une approche paléoécologique. Premiers résultats. In: Fumanal, M.P. and Bernabeu, J. (Eds), Medios Sedimentarios. Cambios ambientales. Hábitat humano. Valencia, ES: Asociación Española Para El Estudio Del Cuaternario, pp. 167-172.

Figueiral, I. (1995) Charcoal analysis and the history of Pinus pinaster (cluster pine) in Portugal. Review of Palaeobotany and Palynology, 89, 441-454.

García-Amorena, I., Gómez Manzaneque, F., Rubiales, J.M., Granja, H.M., Soared de Carvalho, G. and Morla, C. (2007) The Late Quaternary coastal forests of western Iberia: A study of their macroremains. Palaeogeography, Palaeoclimatology, Palaeoecology, 254, 448-461. https://doi.org/10.1016/j.palaeo.2007.07.003

Gauquelin, T., Michon, G., Joffre, R., Duponnois, R., Génin, D., Fady, B. et al (2018) Mediterranean forests, land use and climate change: A social-ecological perspective. Regional Environmental Change, 18(3), 623-636. https://doi.org/10.1007/s10113-016-0994-3

Gernandt, D.S., López, G.G., García, S.O. and Liston, A. (2005) Phylogeny and classification of Pinus. Taxon, 54(1), 29-42.

Granados, M.E., Vilagrosa, A., Chirino, E. and Vallejo, V.R. (2016) Reforestation with resprouter species to increase diversity and resilience in Mediterranean pine forests. Forest Ecology and Management, 362, 231-240. https://doi.org/10.1016/j. foreco.2015.12.020

Gómez-Aparicio, L., Zavala, M.A., Bonet, F.J. and Zamora, R. (2009) Are pine plantations valid tools for restoring Mediterranean forests? An assessment along abiotic and biotic gradients. Ecological Applications, 19(8), 2124-2141. https://doi.org/10.1890/08-1656.1

Heinken, T. (Ed.) (2008) Synopsis der Pflanzengesellschaften Deutschlands 2. Dicrano-Pinion. Sand- und Silikat-Kiefernwälder. Göttingen, DE: Floristisch-soziologische Arbeitsgemeinschaft.

Hennekens, S.M. (2015) Turboveg vol 3 - A gateway to EVA and other databases. In: Chytrý, M., Zelený, D. and Hettenbergerová, E. (Eds.) 58th Annual Symposium of the International Association for Vegetation Science: Understanding broad-scale vegetation patterns - Abstracts. Brno: Masaryk University, p. 152.

Hijmans, R.J. (2020) raster: Geographic Data Analysis and Modeling. R package Version 3.1-5. https://CRAN.R-project.org/package=raster

Hill, M.O. (1979) TWINSPAN: a Fortran Program for Arranging Multivariate Data in an Ordered Two-way Table by Classification of the Individuals and Attributes. New York, NY: Cornell University.

Hill, M.O. and Gauch, H.G. (1980) Detrended correspondence analysis: An improved ordination technique. Vegetatio, 42(1-3), 47-58. https:// doi.org/10.1007/BF00048870

Horvat, I., Glavač, V. and Ellenberg, H. (Eds) (1974). Vegetation Südosteuropas. Stuttgart, DE: G. Fischer. 
Karger, D.N., Conrad, O., Böhner, J., Kawohl, T., Kreft, H., Soria-Auza, R.W. et al (2017) Climatologies at high resolution for the earth's land surface areas. Scientific Data, 4, 170122. [Accessed 20 September 2020].

Karger, D.N. and Zimmermann, N.E. (2019) CHELSA Climatologies at High resolution for the Earth Land Surface Areas. CHELSA V1.2: Technical specification. Swiss Federal Research Institute WSL. Available at http://chelsa-climate.org/about/ [Accessed 20 September 2020]

Kavgacı, A., Šilc, U., Başaran, S., Marinšek, A., Başaran, M.A., Košir, P. et al (2017) Classification of plant communities along postfire succession in Pinus brutia (Turkish red pine) stands in Antalya (Turkey). Turkish Journal of Botany, 41, 299-307.

Knollová, I., Chytrý, M., Tichý, L. and Hájek, O. (2005) Stratified resampling of phytosociological databases: Some strategies for obtaining more representative data sets for classification studies. Journal of Vegetation Science, 16(4), 479-486. https://doi. org/10.1111/j.1654-1103.2005.tb02388.x

Konstantinidis, P., Xofis, P. and Tsiourlis, G. (2012) Syntaxonomy and synecology of thermophilous Mediterranean pines Pinus halepensis Mill. and Pinus brutia Ten. in Greece. Journal of Environmental Protection and Ecology, 3(3), 1416-1423.

Landucci, F., Šumberová, K., Tichý, L., Hennekens, S., Aunina, L., BițăNicolae, C. et al (2020) Classification of the European marsh vegetation (Phragmito-Magnocaricetea) to the association level. Applied Vegetation Science, 23(2), 297-316. https://doi.org/10.1111/avsc.12484

Landucci, F., Tichý, L., Šumberová, K. and Chytrý, M. (2015) Formalized classification of species-poor vegetation: A proposal of a consistent protocol for aquatic vegetation. Journal of Vegetation Science, 26(4), 791-803. https://doi.org/10.1111/jvs.12277

Lavi, A., Perevolotsky, A., Kigel, J. and Noy-Meir, I. (2005) Invasion of Pinus halepensis from plantations into adjacent natural habitats. Applied Vegetation Science, 8(1), 85-92. https://doi.org/10.1111/ j.1654-109X.2005.tb00632.x

Lengyel, A., Chytrý, M. and Tichý, L. (2011) Heterogeneityconstrained random resampling of phytosociological databases. Journal of Vegetation Science, 22(1), 175-183. https://doi. org/10.1111/j.1654-1103.2010.01225.x

Leone, V. and Lovreglio, R. (2004) Conservation of Mediterranean pine woodlands: Scenarios and legislative tools. Plant Ecology, 171(1/2), 221 235. https://doi.org/10.1023/B:VEGE.0000029377.59216.e5

Leuschner, C. and Ellenberg, H. (Eds) (2017) Ecology of Central European forests. Vegetation Ecology of Central Europe I. New York, NY: Springer

Lionello, P. (Ed.) (2012) The climate of the Mediterranean region: From the past to the future, 1st edn. London, UK: Elsevier.

Litvinskaya, S.A. and Postarnak, Y.A. (2002) Syntaxonomy of the Pizunda pine forests at North-Western Caucasus. Yu.D. Kleopov and modern botanical science. Readings on the 100th Anniversary of Yuriy Kleopov's birthday. Kyiv, UA: Phytosociocenter.

Loidi, J. (Ed.) (2017) The Vegetation of the Iberian Peninsula. Vol. 1-2. Cham, $\mathrm{CH}$ : Springer.

López-Sáez, J.A., López-Merino, L., Alba-Sánchez, F., Pérez-Díaz, S., Abel-Schaad, D. and Carrión, J.S. (2010) Late Holocene ecological history of Pinus pinaster forests in the Sierra de Gredos of centra Spain. Plant Ecology, 206, 195-209. https://doi.org/10.1007/s1125 8-009-9634-z

Madrigal-González, J., García-Rodríguez, J.A., Puerto-Martín, A., Fernández-Santos, B. and Alonso-Rojo, P. (2010) Scale-dependent effects of pines on the herbaceous layer diversity in a semi-arid mediterranean ecosystem. Community Ecology, 11(1), 77-83. https://doi. org/10.1556/ComEc.11.2010.1.11

Maestre, F.T. and Cortina, J. (2004) Are Pinus halepensis plantations useful as a restoration tool in semiarid Mediterranean areas? Forest Ecology and Management, 198(1-3), 303-317. https://doi.org/10.1016/j. foreco.2004.05.040

Marcenò, C., Guarino, R., Loidi, J., Herrera, M., Isermann, M., Knollová, I. et al (2018) Classification of European and Mediterranean coastal dune vegetation. Applied Vegetation Science, 21(3), 533-559. https:// doi.org/10.1111/avsc.12379

Marcenò, C., Guarino, R., Mucina, L., Biurrun, I., Deil, U., Shaltout, K. et al (2019) A formal classification of the Lygeum spartum vegetation of the Mediterranean Region. Applied Vegetation Science, 22(4), 593-608.

Martínez, F. and Montero, G. (2004) The Pinus pinea L. woodlands along the coast of South-western Spain: Data for a new geobotanical interpretation. Plant Ecology, 175, 1-18.

Martínez-Jauregui, M., Soliño, M., Martínez-Fernández, J. and Touza, J. (2018) Managing the early warning systems of invasive species of plants, birds, and mammals in natural and planted pine forests. Forests, 9(4), 170. https://doi.org/10.3390/f9040170

Mateus, J. and Queiroz, P. (Eds) (1993) Os estudos de vegetação quaternária em Portugal; contextos, balanço de resultados, perspectivas, O quaternário em Portugal, balanço e perpectivas. Lisbon, PT: Colibri.

Mauri, A., Di Leo, M., de Rigo, D. and Caudullo, G. (2016) Pinus halepensis and Pinus brutia in Europe: Distribution, habitat, usage and threats. In: San-Miguel-Ayanz, J., de Rigo, D., Caudullo, G., Houston Durrant, T. and Mauri, A. (Eds), European Atlas of Forest Tree Species. Luxembourg, LU: Publications Office of the European Union, p. e0166b8+

Mayer, H. and Aksoy, H. (Eds) (1998) Türkiye ormanları [Forests of Turkey]. Bolu, TR: Western Black Sea Forest Research Institute.

Mazzoleni, S. (Ed.) (2004) Recent dynamics of the Mediterranean vegetation and landscape. Chichester, UK: John Wiley \& Sons.

Meddour, R., Meddour-Sahar, O., Zeraia, L. and Mucina, L. (2017) Syntaxonomic synopsis of the forest and tall scrub vegetation of Northern Algeria. Lazaroa, 38(2), 127-163.

Mucina, L., Bültmann, H., Dierßen, K., Theurillat, J.-P., Raus, T., Čarni, A. et al (2016) Vegetation of Europe: Hierarchical floristic classification system of vascular plant, bryophyte, lichen, and algal communities. Applied Vegetation Science, 19, 3-264. https://doi.org/10.1111/ avsc.12257

Mucina, L., Dengler, J., Bergmeier, E., Čarni, A., Dimopoulos, P., Jahn, R. et al (2009) New and validated high-rank syntaxa from Europe. Lazaroa, 30, 267-276.

Mutke, S., Vendramin, G.G., Fady, B., Bagnoli, F. and González-Martínez, S. (2019) Molecular and quantitative genetics of Stone Pine (Pinus pinea). In: Nandwani, D. (Ed.), Genetic diversity in horticultural plants. Switzerland: Springer Nature, Sustainable Development and Biodiversity 22, pp. 61-86.

Oksanen, J., Blanchet, F.G., Kindt, R., Legendre, P., Minchin, P.R., O'Hara, R.B. et al (2019) Vegan: community ecology package. Version 1.17-9. Available at http://cran.r-project.org/web/packages/vegan/index. html [Accessed 20 September 2020]

Ozenda, P. (1975) Sur les étages de végétation dans les montagnes du bassin méditerranéen. Documents de Cartographie Écologique, 16, 1-32.

Panetsos, K.P. (1981) Monograph of Pinus halepensis (Mill.) and P. brutia (Ten.). Annales Forestales, 9(2), 39-77.

Pérez Latorre, A.V., Navas, P., Navas, D., Gil, Y. and Cabezudo, B. (1998) Datos sobre la flora y vegetación de la Serranía de Ronda (Málaga, España). Acta Botánica Malacitana, 23, 149-191.

Pesaresi, S., Biondi, E., Vagge, I., Galdenzi, D. and Casavecchia, S. (2017) The Pinus halepensis Mill. forests in the central-eastern European Mediterranean basin. Plant Biosystems, 151(3), 512-529. https://doi. org/10.1080/11263504.2017.1302514

Peterka, T., Hájek, M., Jiroušek, M., Jiménez-Alfaro, B., Aunina, L., Bergamini, A. et al (2017) Formalized classification of European fen vegetation at the alliance level. Applied Vegetation Science, 20(1), 124-142. https://doi.org/10.1111/avsc.12271

Quézel, P. (1986) The forest vegetation of Turkey. Proceedings of the Royal Society of Edinburgh, 89B, 113-122. 
Quézel, P. (1979) La région méditerranéenne française et ses essences forestières, signification écologique dans le contexte circum-méditerranéen. Forêt Méditerranéenne, 1(1), 7-18.

Quézel, P. (2000) Taxonomy and biogeography of Mediterranean pines (Pinus halepensis and P. brutia). In: Neeman, G. and Trabaud, L. (Ed.) Ecology, Biogeography and Management of Pinus halepensis and P. brutia. Forest Ecosystems in the Mediterranean Basin. Leiden, NL: Backhuys, pp. 1-12.

Quézel, P. and Barbéro, M. (1986) Aperçu syntaxinomique sur la connaissance actuelle de la classe des Quercetea ilicis au Maroc. Ecologia Mediterranea. 12(3-4), 105-111.

Quézel, P. and Barbéro, M. (1988) Signification phytoécologique et phytosociologique des peuplements naturels de Pin de Salzmann en France. Ecologia Mediterranea, 14(1-2), 41-63.

Quézel, P., Barbéro, M. and Akman, Y. (1978) L'Interpretation phytosociologique des groupements forestiers dans le Bassin Mediterraneen Oriental. Documents Phytosociologiques, 2, 329-352.

Quézel, P., Barbéro, M. and Akman, Y. (1980) Contribution à l'étude de la végétation forestière d'Anatolie septentrionale. Phytocoenologia, 8 , 365-519.

Quézel, P., Barbéro, M. and Benabid, A. (1987) Contribution a l'étude des groupements forestiers et pré-forestiers du Haut Atlas oriental (Maroc). Ecologia Mediterranea, 13(1-2), 107-117.

Quézel, P., Barbéro, M., Benabid, A., Loisel, R. and Rivas-Martínez, S. (1988) Contribution à l'étude des groupements pré-forestiers et des matorrals rifains. Ecologia Mediterranea, 14(1-2), 77-122.

Quézel, P., Barbéro, M., Benabid, A. and Rivas-Martínez, S. (1992) Contribution à l'étude des groupements forestiers et pré-forestiers du Maroc oriental. Studia Botanica, 10, 57-90.

Quézel, P. and Pamukçuoğlu, A. (1973) Contribution à l'étude phytosociologique et bioclimatique de quelques groupements forestiers du Taurus. Feddes Repertorium, 84(3), 185-229.

Rodwell, J.S., Schaminée, J.H.J., Mucina, L., Pignatti, S., Dring, J. and Moss, D. (2002) The diversity of European vegetation: An overview of phytosociological alliances and their relationships to EUNIS habitats. Wageningen, NL: National Centre for Agriculture, Nature Management and Fisheries.

R Core Team (2019) A language and environment for statistical computing. Vienna, AT: R Foundation for Statistical Computing. Available at http://www.R-project.org/ [Accessed 20 September 2020]

Rivas-Martínez, S. (1974) La vegetación de la clase Quercetea ilicis en España y Portugal. Anales del Instituto Botánico A. J. Cavanilles, 31(2), 205-259.

Rivas-Martínez, S. (1981) Les étages bioclimatiques de la végétation de la Péninsule Ibérique. Actas III Congreso OPTIMA. Anales del Jardín Botánico de Madrid, 37(2), 251-268.

Rivas-Martínez, S. (Ed.) (2011) Mapa de series, geoseries y geopermaseries de vegetación de España [Memoria del mapa de vegetación potencial de España, 2011]. Part II. Itinera Geobotanica, 18, 425-800.

Rivas-Martínez, S., Costa, M. and Izco, J. (1986) Sintaxonomía de la clase Quercetea ilicis en el Mediterráneo occidental. Notiziario Fitosociologico, 19(1), 71-98.

Rivas-Martínez, S. and Rivas Sáenz, S. (2019) Worldwide Bioclimatic Classification System. Available at http://www.globalbioclimatics.org [Accessed 1 June 2019]

Rundel, P.W., Arroyo, M.T.K., Cowling, R.M., Keeley, J.E., Lamont, B.B. and Vargas, P. (2016) Mediterranean biomes: Evolution of their vegetation, floras, and climate. Annual Review of Ecology, Evolution, and Systematics, 47(1), 383-407. https://doi.org/10.1146/annurev-ecols ys-121415-032330

Scarascia-Mugnozza, G., Oswald, H., Piussi, P. and Radoglou, K. (2000) Forests of the Mediterranean region: Gaps in knowledge and research needs. Forest Ecology and Management, 132(1), 97-109. https://doi.org/10.1016/S0378-1127(00)00383-2
Sokal, R.R. and Rohlf, F.J. (Eds) (1995) Biometry: The Principles and Practice of Statistics in Biological Research, 3rd edn. New York, NY: Freeman.

Theurillat, J.-P., Willner, W., Fernández-González, F., Bültmann, H., Čarni, A., Gigante, D. et al (2021) International Code of Phytosociological Nomenclature. 4th edition. Applied Vegetation Science, 24, e12491. https://doi.org/10.1111/avsc.12491

Tichý, L. (2002) JUICE, software for vegetation classification. Journal of Vegetation Science, 13(3), 451-453. https://doi. org/10.1111/j.1654-1103.2002.tb02069.x

Tichý, L. and Chytrý, M. (2006) Statistical determination of diagnostic species for site groups of unequal size. Journal of Vegetation Science, 17(6), 809-818. https://doi.org/10.1111/j.1654-1103.2006.tb02504.x

Tichý, L., Chytrý, M. and Landucci, F. (2019) GRIMP: A machine-learning method for improving groups of discriminating species in expert systems for vegetation classification. Journal of Vegetation Science, 30(1), 5-17. https://doi.org/10.1111/jvs.12696

Timbal, J., Bonneau, M., Landmann, G., Trouvilliez, J. and BouhotDelduc, L. (Eds) (2005) European non-boreal conifer forests. Ecosystems of the World (6): Coniferous Forests. Amsterdam, NL: Elsevier.

Vural, M., Akman, Y. and Quézel, P. (1999) Contribution a l'étude de la vegetation forestière du Taurus central: analyse phyto-écologique d'un transect sud-nord, entre Silifke et Karaman. Fitosociologia, 36(1), 3-21.

Willner, W., Jiménez-Alfaro, B., Agrillo, E., Biurrun, I., Campos, J.A., Čarni, A. et al (2017a) Classification of European beech forests: A Gordian Knot? Applied Vegetation Science, 20(3), 494-512. https:// doi.org/10.1111/avsc.12299

Willner, W., Kuzemko, A., Dengler, J., Chytrý, M., Bauer, N., Becker, T. et al (2017b) A higher-level classification of the Pannonian and western Pontic steppe grasslands (Central and Eastern Europe). Applied Vegetation Science, 20(1), 143-158. https://doi.org/10.1111/ avsc. 12265

Zavala, M.A. and Zea, E. (2004) Mechanisms maintaining biodiversity in Mediterranean pine-oak forests: Insights from a spatial simulation model. Plant Ecology, 171(1-2), 197-207. https://doi.org/10.1023/ B:VEGE.0000029387.15947.b7

\section{SUPPORTING INFORMATION}

Additional supporting information may be found online in the Supporting Information section.

Appendix S1 Databases, countries and plot sizes used for the analysis. Appendix S2 Expert system (script v. 2020-09-22) for classification of the phytosociological alliances and informal groups of plant communities of the Mediterranean thermophilous pine forests.

Appendix S3 Diagnostic, constant and dominant species for the accepted clusters based on TWINSPAN classification.

Appendix S4 Diagnostic, constant and dominant species for the alliances and informal groups of plant communities classified by the expert system.

How to cite this article: Bonari G, Fernández-González F, Çoban S, et al. Classification of the Mediterranean lowland to submontane pine forest vegetation. Appl Veg Sci.

2021;24:e12544. https://doi.org/10.1111/avsc.12544 